\title{
Jatrophane and rearranged jatrophane-type diterpenes: biogenesis, structure, isolation, biological activity and SARs (1984-2019)
}

\author{
Maryam Fattahian • Mustafa Ghanadian · Zulfiqar Ali • Ikhlas A. Khan
}
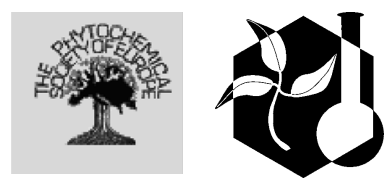

Received: 4 September 2019/Accepted: 29 February 2020/Published online: 13 April 2020

(C) Springer Nature B.V. 2020

\begin{abstract}
Diterpene compounds specially macrocyclic ones comprising jatrophane, lathyrane, terracinolide, ingenane, pepluane, paraliane, and segetane skeletons occurring in plants of the Euphorbiaceae family are of considerable interest in the context of natural product drug discovery programs. They possess diverse complex skeletons and a broad spectrum of therapeutically relevant biological activities including anti-inflammatory, anti-chikungunya virus, antiHIV, cytotoxic, and multidrug resistance-reversing activities as well as curative effects on thrombotic diseases. Among macrocyclic diterpenes of Euphorbia, the discovery of jatrophane and modified jatrophane diterpenes with a wide range of structurally unique polyoxygenated polycyclic derivatives and as a new class of powerful inhibitors of P-glycoprotein has
\end{abstract}

Electronic supplementary material The online version of this article (https://doi.org/10.1007/s11101-020-09667-8) contains supplementary material, which is available to authorized users.

M. Fattahian · M. Ghanadian

School of Pharmacy and Pharmaceutical Sciences, Isfahan

University of Medical Sciences, Isfahan, Iran

M. Ghanadian $(\bowtie) \cdot$ Z. Ali $(\bowtie) \cdot$ I. A. Khan

National Center for Natural Products Research, School of

Pharmacy, University of Mississippi, University,

MS 38677, USA

e-mail: ghannadian@gmail.com

Z. Ali

e-mail: zulfiqar@olemiss.edu opened new frontiers for research studies on this genus. In this review, an attempt has been made to give in-depth coverage of the articles on the naturally occurring jatrophanes and rearranged jatrophane-type diterpenes isolated from species belonging to the Euphorbiaceae family published from 1984 to March 2019, with emphasis on the biogenesis, isolation methods, structure, biological activity, and structureactivity relationship.

Keywords Natural products · Euphorbia · Jatrophane diterpenes · Rearranged Jatrophanes · Chromatography $\cdot$ Multidrug resistance

$\begin{array}{ll}\text { Abbreviations } \\ \text { ABCB1 } & \begin{array}{l}\text { ATB-Binding Cassette Sub-Family B } \\ \text { Member 1 }\end{array} \\ \text { Bel-7402 } & \begin{array}{l}\text { Hepatoma cell line } \\ \text { BGC-823 }\end{array} \\ \text { Human gastric carcinoma cell line } \\ \text { Caov-4 } & \text { Ovarian cancer cell line } \\ \text { CBS } & \text { Casbene synthase } \\ \text { CC } & \text { Column chromatography } \\ \text { CHIK } & \text { Chikungunya virus } \\ \text { CNS } & \text { Central nervous system } \\ \text { COLO320 } & \text { Colon adenocarcinoma cell line } \\ \text { CsA } & \text { Cyclosporine A } \\ \text { DCCC } & \text { Droplet } \\ & \text { countercurrent chromatography } \\ \text { EC } & \text { Half maximal effective concentration } \\ \text { FAR } & \text { Fluorescence activity ratio }\end{array}$




\begin{tabular}{|c|c|c|}
\hline FICi & $\begin{array}{l}\text { Fractional inhibitory concentration } \\
\text { index }\end{array}$ & $\begin{array}{l}\text { Glioblastoma brain cell line } \\
\text { Semliki forest virus }\end{array}$ \\
\hline FIX & Fractional inhibitory index & Seletivity index \\
\hline GGPP & Geranylgeranyl pyrophosphate & Sindbis virus \\
\hline \multirow[t]{3}{*}{$\mathrm{GI}_{50}$} & concentration of the anti-cancer drug & Single nucleotide polymorphism \\
\hline & that inhibits the growth of cancer cells & Thin layer chromatography \\
\hline & By $50 \%$ & Tariquidar \\
\hline \multirow[t]{2}{*}{ GIRK } & G protein-coupled inwardly & Tropomyosin receptor kinase \\
\hline & rectifying potassium & Vacuum liquid chromatography \\
\hline GTP & Guanosine triphosphate & \\
\hline HAART & Highly active antiretroviral therapy & \\
\hline HEK293 & Human embryonic kidney cell line & Introduction \\
\hline Hela & Human cervical carcinoma cells & \\
\hline HPLC & $\begin{array}{l}\text { High-performance liquid } \\
\text { chromatography }\end{array}$ & $\begin{array}{l}\text { Natural products are comprised of a large number of } \\
\text { structurally complex molecules, the structural diver- }\end{array}$ \\
\hline $\mathrm{IC}_{50}$ & Half maximal inhibitory concentration & sity of which sometimes far exceeds the abilities of \\
\hline $\mathrm{ID}_{50}$ & Median infectious dose & chemists and their equipment within the laboratories. \\
\hline $\mathrm{LC}_{50}$ & Median lethal dose & In addition to the fascinating diverse structures, many \\
\hline LPS & Lipopolysaccharide & natural compounds possess intriguing biological prop- \\
\hline MCF-7 & Breast cancer cell line & erties. Building blocks of natural origin are being used \\
\hline $\begin{array}{l}\text { MDA- } \\
\text { MB-231 }\end{array}$ & Breast tumor cells & $\begin{array}{l}\text { as a plentiful source of lead compounds for drug } \\
\text { discovery. Euphorbiaceae family composed of five }\end{array}$ \\
\hline MDR & Multidrug resistance & subfamilies, 49 tribes, 317 genera, and about 8000 \\
\hline MFS & Major facilitator superfamily & species, is one of the biggest families with probably \\
\hline $\mathrm{MIC}_{80}$ & $\begin{array}{l}\text { Minimum inhibitory concentration (For } \\
80 \% \text { ) }\end{array}$ & $\begin{array}{l}\text { the highest species richness in many habitats (Webster } \\
\text { 1986). Exposure to a large range of habitats predis- }\end{array}$ \\
\hline MPLC & $\begin{array}{l}\text { Medium pressure } \\
\text { liquid chromatography }\end{array}$ & $\begin{array}{l}\text { posed Euphorbia species to unavoidable high muta- } \\
\text { tion loads caused by stressful habitats. The presence of }\end{array}$ \\
\hline MRP & Multidrug resistance protein & environmental stimuli had necessitated the develop- \\
\hline MTB & Methyl-tert-butyl ether & ment of rich storage of defensive secondary metabo- \\
\hline MTT & $\begin{array}{l}\text { 3-(4,5-Dimethylthiazol-2-Yl)-2,5- } \\
\text { diphenyltetrazolium bromide }\end{array}$ & $\begin{array}{l}\text { lites (Mwine and Van Damme 2011). The botanical } \\
\text { name "Euphorbia" derives from the Greek "Euphor- }\end{array}$ \\
\hline NADH & $\begin{array}{l}\text { Nicotinamide adenine dinucleotide } \\
\text { factor }\end{array}$ & $\begin{array}{l}\text { bius" in honour to the physician of Mauritania, who is } \\
\text { assumed to have used in his treatment a certain plant }\end{array}$ \\
\hline NCI-H460 & Non-small cell lung carcinoma cell line & (Euphorbia resinifera) with a milky latex (Appendino \\
\hline $\mathrm{NF}-\kappa \mathrm{B}$ & Nuclear factor & and Szallasi 1997). Moreover, this plant family is \\
\hline NGF & Nerve growth factor & known also as "spurge" derived from the Latin \\
\hline NP & Normal phase & "expurgare", which means "to cleanse" referring to \\
\hline OVCAR-3 & Ovarian carcinoma cell line & the early traditional application of these plants as \\
\hline $\mathrm{PE}$ & Petroleum ether & purgative medication (Burkill 1994). Euphorbiaceae \\
\hline P-gp & P-glycoprotein & species had played an important role in traditional \\
\hline PKC & Protein kinase $\mathrm{C}$ & ethnomedicine as mentioned in the Greek and Roman \\
\hline PTX & Paclitaxel & medical literature for the treatment of toothache, to \\
\hline $\mathrm{RP}$ & Reverse phase & remove warts, as purgatives, and in asthma and \\
\hline $\mathrm{RR}$ & Relative resistance & bronchial catarrh (Lawant and Winthagen 2002). They \\
\hline Rho123 & Rhodamine-123 & are also part of different herbal remedies used in \\
\hline SAR & Structure activity relationship & traditional Chinese medicines and ayurvedic medicine \\
\hline SGC-7901 & Human gastric carcinoma cell line & for similar indications (Kapoor 2017; Liang et al. \\
\hline
\end{tabular}


2009). Over the last decades, several Euphorbiaceae constituents have successfully been employed in clinical trials or applied as lead structures for the development of novel drugs. Species of this family are prolific producers of unique diterpenoids (Singla and Kamla 1990) of great biomedical relevance (Evans and Taylor 1983), the promising biological properties of which have attracted interests of phytochemists to the isolation of Euphorbiaceae constituents. One of the largest chemical classes isolated from the milky latices of Euphorbiaceae species is macrocyclic diterpenes based on jatrophane, lathyrane, terracinolide, ingenane, pepluane, paraliane, and segetane skeletons, many of which show interesting pharmacological properties. When 'jatrophone', the first jatrophanetype diterpene, isolated by Kupchan and co-workers in 1970 from Jatropha gossypiifolia L. as a natural product with significant antiproliferative effects against human tumor cell lines, the biological and chemical interest in the jatrophane structures greatly increased (Kupchan et al. 1970). Modified jatrophanes consist of "segetanes", "paralianes", “pepluanes", and "terracinolides". "Euphoractanes" being occasionally considered as modified jatrophanes or modified lathyrane skeletons, were a black box for decades and there was no biosynthesis or chemical conversion evidence to support or oppose different biogenesis proposals. In this regard, the recently published article by Wang et al. (2019) has mentioned the proposal suggested by Haiming et al. (2008) in which it had been claimed that euphoractane skeletons come from macrocyclic jatrophanes. Subsequently, Wang et al. in 2019 have certainly demonstrated that euphoractane skeletons are obtained by the treatment of lathyranetype diterpene (Euphorbia Factor L1) with $\mathrm{BF}_{3} \cdot \mathrm{ET}_{2} \mathrm{O}$ in ethyl acetate at room temperature and it has confirmed the biogenesis relationship between the euphoractanes and lathyranes by chemical conversion method for the first time. Hereupon, euphoractanes are not considered as modified jatrophane skeletons (Wang et al. 2019). Vasas and Hohmann (2014) have published a worthwhile review article of the represented papers on all diterpenoids isolated from Euphorbia between 2008 and 2012, parts of which include jatrophane and modified jatrophane diterpenes (Vasas and Hohmann 2014). Moreover, another comprehensive review article has been published by Shi et al. (2008) of the papers written on the chemical and pharmacological aspects of the plants in genus
Euphorbia over the past few decades (Shi et al. 2008). Meanwhile, the vacancy of a review article intensely focusing on jatrophane diterpenes was sensated. Therefore, this present review article has been aimed at giving in depth coverage of the papers published from 1984 to March 2019 particularly on the jatrophanes and rearranged jatrophane-type diterpenes, with emphasis on their biogenesis, isolation, structure, biological activity and structure activity relationship.

\section{Biogenesis}

As demonstrated in Fig. 1, two mechanistically different biogenetic pathways are possible for the biosynthesis of diterpenes, leading either to the phytanes such as abietanes, kauranes, atisanes, etc. or to the casbene derived diterpenes including casbanes, jatrophanes, tiglianes, etc. (Appendino et al. 2000).

Casbene is considered as a precursor for different macrocyclic and polycyclic diterpenes including those of the jatrophane-, casbane-, lathyrane-, tigliane-, ingenane- and daphnane- type (Breitmaier 2006).

Biosynthesis of casbene derived diterpenes, commences from GGPP; the diphosphate group is cleaved from GGPP affording requisite delocalized cation, which interacts with the $\mathrm{C}-(14,15)$ terminal double bond and transformed to cembrene intermediate with C-15 tertiary carbocation undergoing additional cyclization and rearrangements to form a diversity of the carbon skeletons outlined in Fig. 2 (Breitmaier 2006; Nakano et al. 2012; Rinner 2015; Robinson and West 1970). Finally, the cyclopropane ring is formed via a nonclassical carbocation (a corner-protonated cyclopropane) by proton loss of cembrene intermediate, delivering casbene. The whole sequence is catalyzed by a single enzyme, called casbene synthase (E1) (Fig. 3) (Dewick 2002; Kirby et al. 2010). A second ring closure between C- 6 and C- 10 delivers the precursor of natural products of the lathyrane family and a third ring closure between C-5 and C-14 affords the tigliane skeleton an intermediate in the hypothetical biogenetic route toward phorbol (Kinghorn et al. 2011).

From casbene, the biosynthetic route to macrocyclic and polycyclic diterpenoids is poorly understood but is thought to proceed through intermediates 
Fig. 1 Biogenesis route from GGPP to phytanes and casbene derived diterpenes<smiles>CC(C)=CCC/C(C)=C/CC/C(C)=C/CC/C(C)=C/COc1ccccc1</smiles>

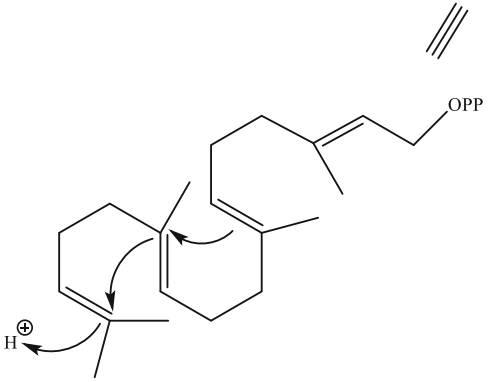

phytanes (abietanes, pimaranes, atisanes, kauranes, etc.)

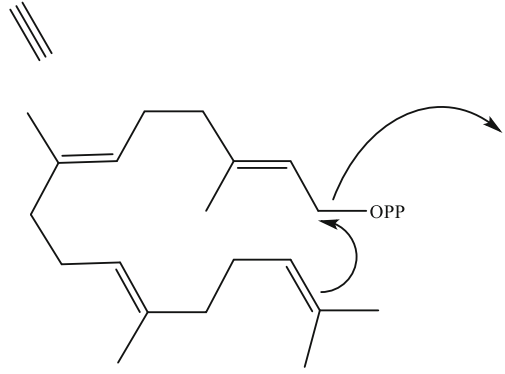

casbene derived diterpenes (casbanes, jatrophanes, lathyranes, tiglianes, etc.)

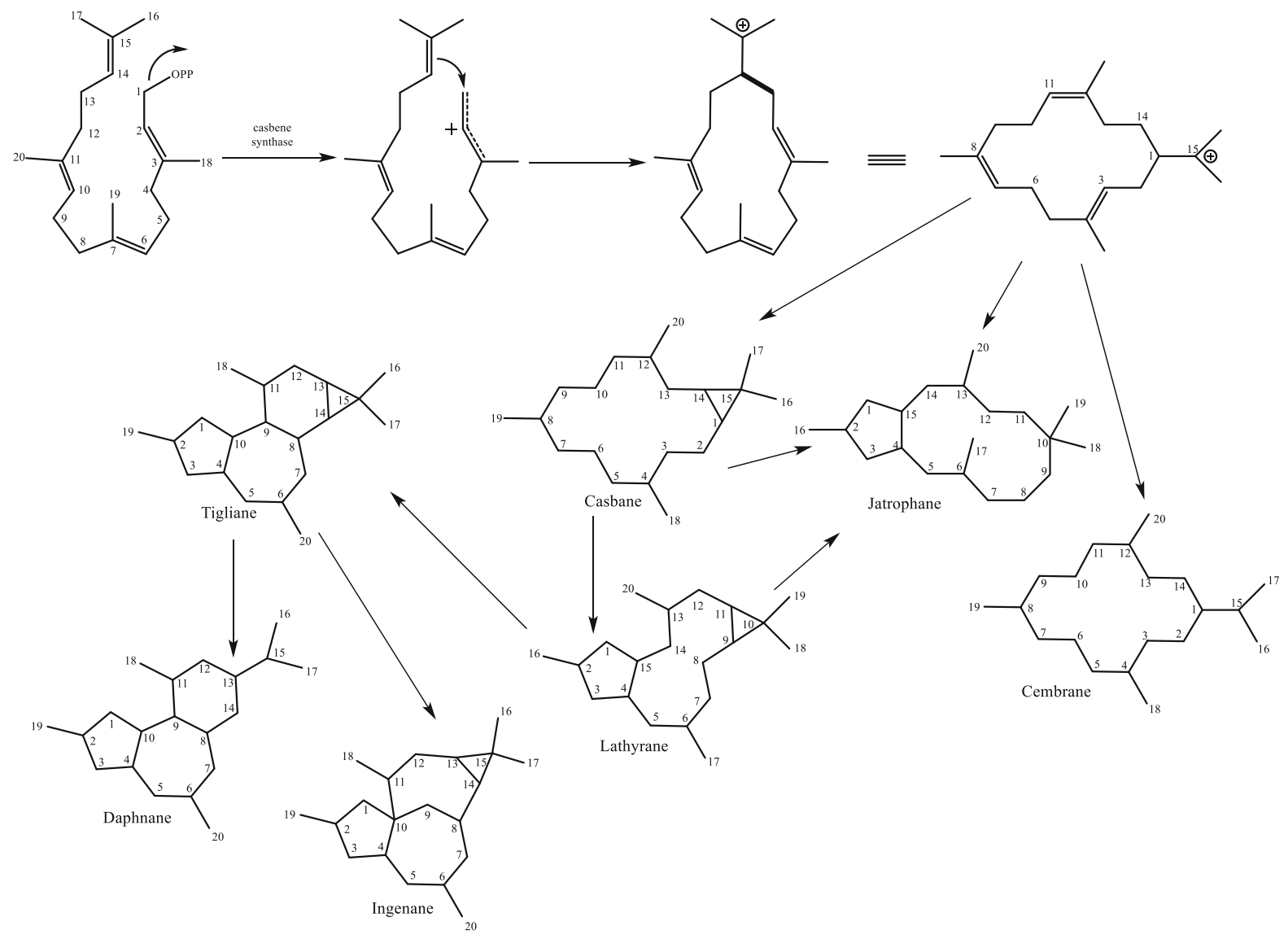

Fig. 2 Biogenesis route of macrocyclic and polycaclic derived from Casbene

such as jolkinol $\mathrm{C}$ via cytochrome P450-catalyzed oxidations and possibly a short-chain alcohol dehydrogenase (ADH) (Fig. 4). This cyclization requires the activity of two CYP450s to form an intermediate '6-hydroxy-5,9-diketocasbene' including one of the 


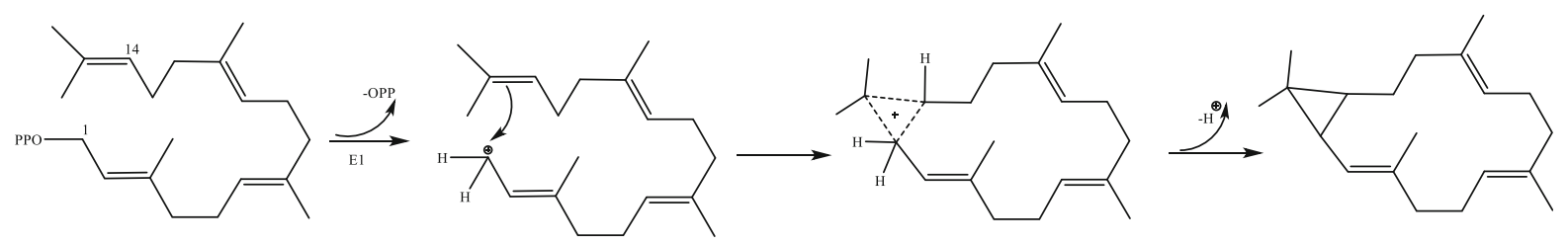

Fig. 3 Sequence of transforming the cembrene to casbene

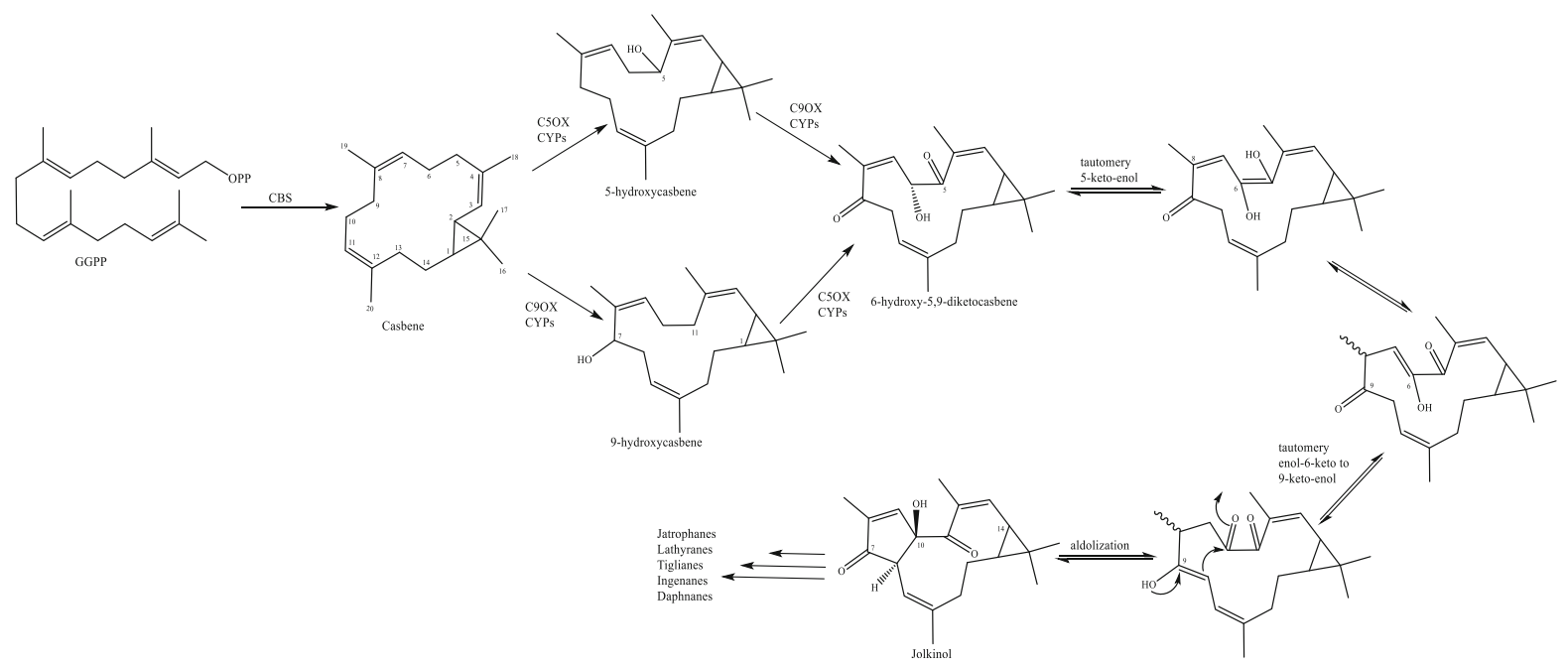

Fig. 4 Proposed pathway for the production of macrocyclic diterpenes in detail

tautomers (9-hydroxy-5,6-diketocabene) may undergo aldolization (King et al. 2016).

Jatrophanes and cyclojatrophanes

Three different biogenetic mechanisms for the formation of the jatrophane framework have already been reported (Fig. 2). Jatrophane is a bicyclic pentadecane skeleton (Fig. 5) without the cyclopropane ring (Evans and Taylor 1983) which is biosynthesized either directly from above mentioned cembrene cation

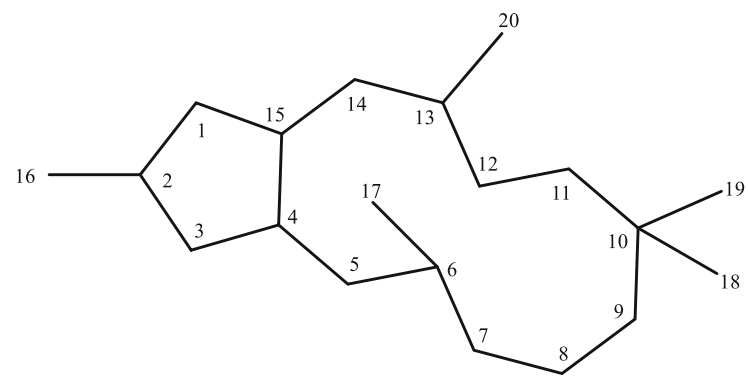

Fig. 5 Jatrophane skeleton through Wagner-Meerwein rearrangements or through the more likely casbene pathway. In the biosynthesis of jatrophane from casbene rout, casbene precursor is formed first, followed by the opening of the cyclopropane ring, and then closure of the five-membered ring between $\mathrm{C}-6$ and $\mathrm{C}-10$ to form jatrophane core (Fig. 2) (Adolf and Hecker 1977; Lanzotti 2013). According to a different point of view, jatrophanes may be derived from lathyranes by cyclopropane ring being opened (Appendino 2016; Lanzotti 2013).

Final closure of the five-membered ring between C-6 and C-10 would accomplish the biogenetic route toward the jatrophane skeleton. Further functionalization leads to a huge class of natural products with different oxygenation states and stereochemical features. Only a few explanations concerning the biosynthesis of natural products, which are considered to arise from the jatrophane skeleton, have been reported.

In a study reported by Pattenden and Smithies (1996) the mechanism of cyclopropane ring opening in casbene was investigated. Using several radicalmediated reactions with casbene, they found a number of products which are in agreement with compounds 
found as metabolites, such as those from the cembrane family. The detailed mechanism, however, is not yet clear. The participation of a "casbene synthetase", which needs a divalent cation such as magnesium, is also discussed in the biosynthetic pathway of casbene (Dueber et al. 1978).

The skeleton-type $1(15 \rightarrow 14)$ abeo-jatrophane with $6 / 12$ membered ring system differs from the jatrophane skeleton with 5/12 membered ring system in the migration of $\mathrm{C}-15$ in its original place $\mathrm{C}-1-\mathrm{C}-15$ single bond in jatrophane parent framework to another position. C-1 position remained unchanged and is connected to $\mathrm{C}-14$ in the final structure instead of C-15 in parent structure, leads to conversion of the fivemember ring to a six-member ring. The numbering of the structure is also retained unchanged in the new abeo scaffold. Marco et al. reported a pinacol-type rearrangement [13-14] $\alpha$-ketol (pinacolic) of an oxidized jatrophane in positions 14 and 15 to explain these five to six-member ring extension (Fig. 6) (Marco et al. 1998).

Another class of jatrophanes is 12,17-cyclojatrophanes with 5/8/8 membered ring system. A proposed biogenetic pathway for the rare 12,17-cyclojatrophanes has been illustrated in Fig. 7. It appears that Jatrophanes (119-122) are biogenetically interrelated. In this regards, the 11,12-epoxidation of a favorable $\Delta 6(17), \Delta 11$-jatrophane precursor results in epoxiwelwitschene whose epoxide ring can undergo a nucleophilic attack in two different ways. Epoxy ringopening by the 15-hydroxyl group nucleophilic attack causes the formation of a tetrahydrofuran ring, leading to welwitschene (route b) with 12,17-cyclojatrophane structure. In a second way, the attack by the 6(17)exomethylene gives rise to a 12,17-transannular cyclization (route a). This 12,17-cyclojatrophane intermediate would then subsequently go through dehydration at $\mathrm{C}-11$. Epoxidation of the resulting double bond and oxidation at C-2 affords salicifoline which is another rare structural feature of euphowelwitschine A and has been isolated from Euphorbia salicifolia to date (route c) (Hohmann et al. 2001b). Euphowelwitschines A and B supposed to be formed via epoxide ring-opening by the free hydroxyl at C-15 (route d) (Fig. 7) (Reis et al. 2015). This 12,15-ether bridge is not common in macrocyclic jatrophanes; the only compounds that have such functionality were isolated exclusively from Euphorbia helioscopia (Kosemura et al. 1985; Lu et al. 2008; Yamamura et al. 1989).

Another type of cyclojatrophane is 9,13-cyclojatrophane with an architecturally novel (5.9.5) tricyclic framework named jatrophatrione. It was isolated from the chloroform extract of Jatropha macrorhiza roots. It was recognized by the University of Arizona team as a tumor-inhibitory agent being particularly active toward the P-388 (3PS) lymphocytic leukemia assay (Torrance et al. 1976). An isomeric compound, citlalitrione, has subsequently been reported from Jatropha dioica (Villarreal et al. 1988), but its bioactivity has not been evaluated. Jatrophatrione may be derived in nature from the bicyclic precursor illustrated its formation through the biosynthetic route of casbene origin as discussed before (Fig. 8) (Torrance et al. 1976).

Further functionalization leads to a huge class of natural products with different oxygenation states and stereochemical features. Jatrophane diterpenes occur generally in form of polyesters. They are mainly polyacylated derivatives whose number of ester moieties is ranging between three (guyonianin E) (Hegazy et al. 2010) and eight (esulatin H) (Vasas et al. 2011). The acyl residues are frequently acetyl, propionyl, butanoyl, isobutanoyl, 2-methylbutanoyl, angeloyl, tigloyl, benzoyl, nicotinoyl, or rarely cinnamoyl. Depending on their substitution, jatrophanes may
Fig. 6 Pinacol-type rearrangement of a jatrophane to $1(15 \rightarrow 14)$ abeo-jatrophane skeleton

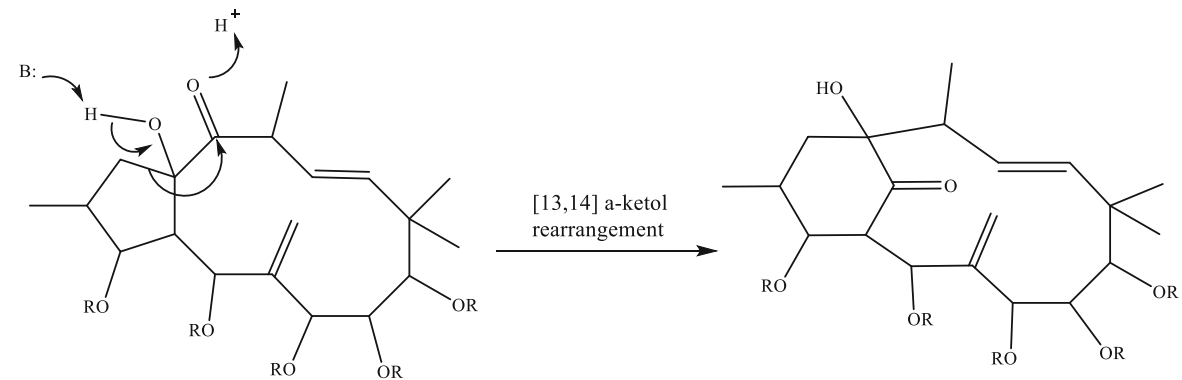




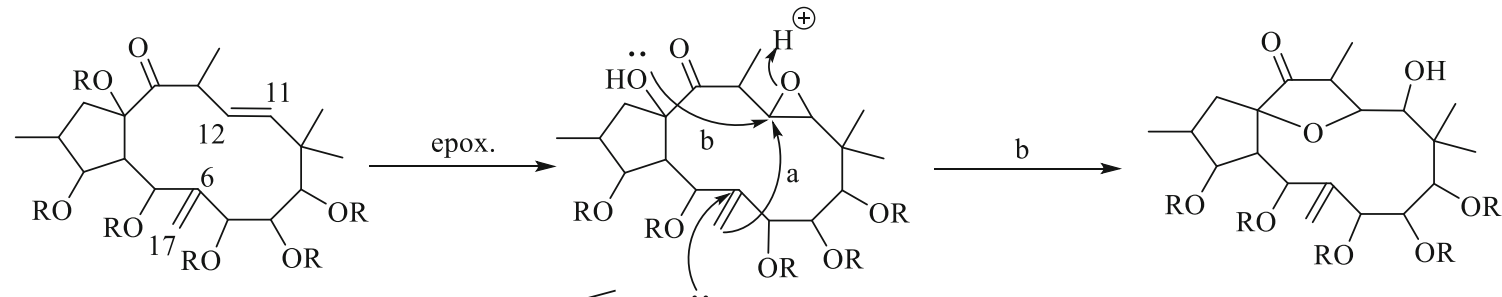

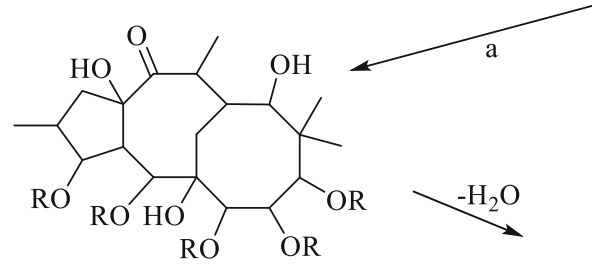

12,17-cyclojatrophane

$\mathrm{H}_{2} \mathrm{O}$

Epoxiwelwitschene

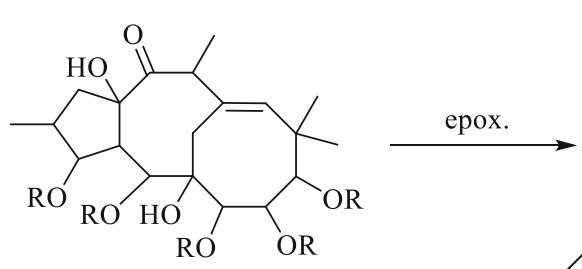

Welwitschene

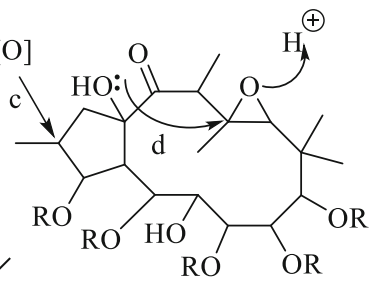

d

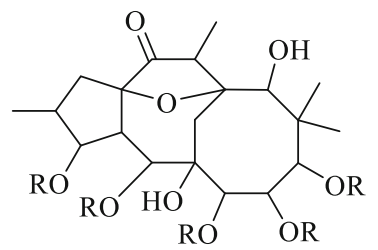

Euphowelwitschines

$\mathrm{A}$ and $\mathrm{B}$

Fig. 7 Proposed biogenetic pathway for 12,17-cyclojatrophanes

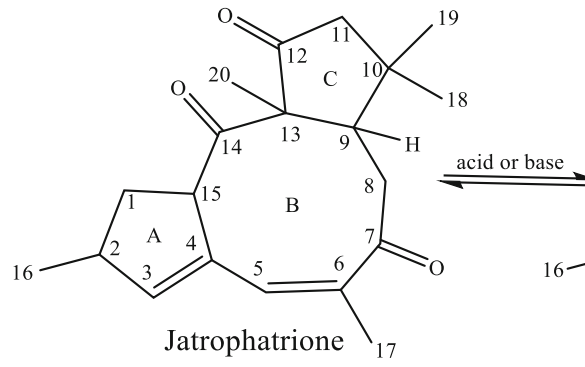

Fig. 8 Proposed biogenetic pathway for 9,13-cyclojatrophanes

have 5 to 10 chiral centers and since the configuration of the carbons is variable, jatrophanes do not form a stereochemically homogeneous series. Other structural variabilities arised from the number and position of the double bonds, the nature and number of oxygen functions (hydroxy, keto, epoxy, ether or ester groups) and the configuration of the diterpene core.
Segetane diterpenoids

The segetane diterpenoids are the main constituents of Euphorbia segetalis (Jakupovic et al. 1998a), a species that the name of the entire skeletal class had originated from it. Segetanes had been isolated from E. peplus (Wan et al. 2016a) and E. portlandica (Madureira et al. 2006) and E. paralias grown in Turkey (Öksüz et al. 1997), Spain (Jakupovic et al. 1998c), Egypt 
(Abdelgaleil et al. 2001), and Italy (Barile and Lanzotti 2007). They are characterized by a modified jatrophane skeleton comprising a bicyclo [4.3.1] undecane ring system which could have up to nine chiral centers.

Segetane originates from an appropriate jatrophane skeleton through cyclization steps that occurred on the unprecedented tricyclic skeleton found for pre-segetanin as a possible intermediate supposed by Jakupovic (Jakupovic et al. 1998a) (Fig. 9). In general, segetanes can be derived from an epoxidized jatrophane in $\Delta 6(17)$. Extant vinyl alcohol is followed by a complete cycle expansion which can be illustrated by an enzymatic epoxidation $\Delta 6(17)$ followed by an acidcatalyzed opening of this epoxide ring (Fig. 9).

Unlike the aforementioned biosynthesis proposed by Barile et al. (2007) and previously by Jakupovic et al. (1998a, b, c) that the segetane tetracyclic skeleton was formed by a two-steps cyclization of jatrophane derivative, Wan et al. (2016b) found that an intermediate with four double bonds is a precursor of segetanes. Through this biosynthetic pathway, the intermediate bearing four double bonds is formed by an elimination reaction on a proper jatrophane; after that this intermediate can be transformed into a segetane via a Diels-Alder reaction in the presence of a Lewis base and/or a Lewis acid (Fig. 10). The study of the generalization of this reaction proved that the presence of a carbonyl at C-9 and lack of substitution at C-8 are indispensable to the formation of a segetane skeleton from a jatrophane skeleton (Wan et al. 2016b).

Pepluane and paraliane

Both pepluane and paraliane diterpenes are based on a fused tetracyclic core originated from further rearrangements of an proper jatrophane (Fig. 11) (Jakupovic et al. 1998c). The paraliane skeletones isolated for the first time from Euphorbia paralias in 1998 are rare 5/6/5/5 tetracyclic systems, probably formed through a transannular ring-closing reaction of the jatrophane diterpene (a jatropha-6(17),12-diene) resulting in a $5 / 6 / 5 / 5$-ring system. This hypothesis is supported by the fact that jatrophanes are systematically co-isolated (Zhou et al. 2016). The introduction of primary alcohol on gem-dimethyl followed by a complete cycle expansion results in the formation of the peplus skeleton $(5 / 6 / 5 / 6)$. The acetylated vicinal diol (C-8 and C-9) found in all known pepluans, can be explained by an enzymatic epoxidation in $\Delta 8(9)$ followed by an opening of the epoxide as shown in Fig. 11 (Hohmann et al. 1999a; Jakupovic et al. 1998c).

Terracinolide diterpenes

Terracinolide is another diterpene skeleton based on a modified jatrophane skeleton. These compounds display a 17-ethyl bis-homojatrophane $\left(\mathrm{C}_{22}\right)$ framework, a skeleton previously found in E. terracina diterpenes (Marco et al. 1996), that gave the name to the entire skeletal class. The terracinolide skeleton bearing an additional two-carbon segment bound to $\mathrm{C}-17$ in the framework of a $\delta$-lactone ring. This attachment of a two-carbon fragment to C-17 could arise from the opening of a 5,17-epoxide by nucleophilic attack on a $\mathrm{C}_{2}$ unit (acetate or malonate) followed by cyclization with a proximate hydroxyl group to give a $\delta$-lactone ring (Marco et al. 1997) (Fig. 12).

\section{Structures of isolated compounds}

A tremendous number of jatrophane diterpenoids including twelve-membered ring jatrophanes, 5/8/8 fused ring systems, rearranged polycyclic jatrophanes, and terracinolides have been isolated and reported from 1984 to 2019 which are arranged in order of chemical structure in Table 1.

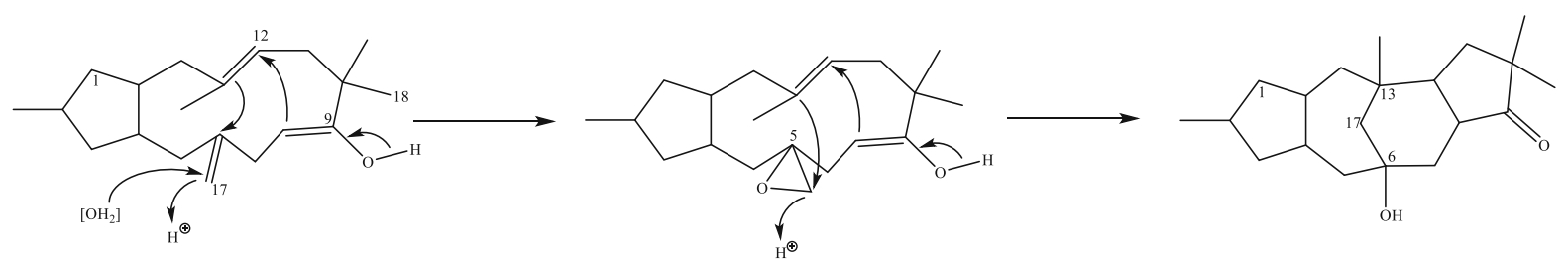

Fig. 9 First proposed biogenesis route for segetane diterpenoids 
Fig. 10 Second proposed biogenesis route for segetane diterpenoids
Fig. 11 Biogenesis route of Pepluane and paraliane diterpenoids
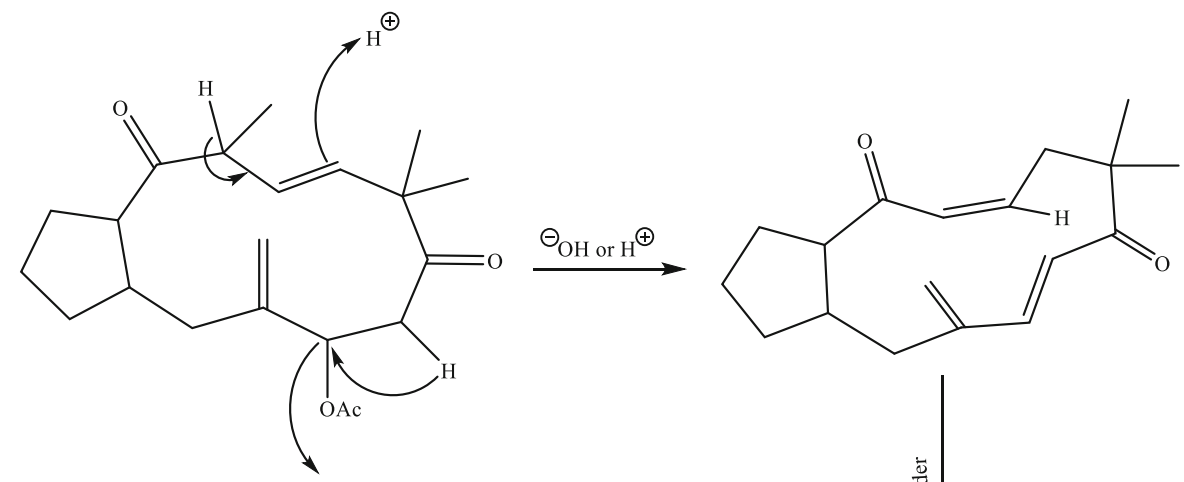

\}

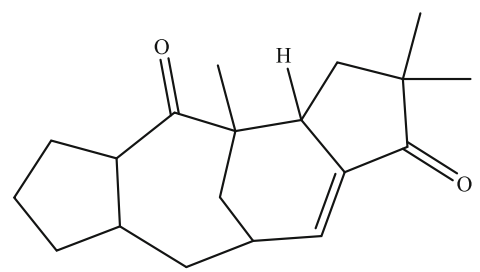

isomerization

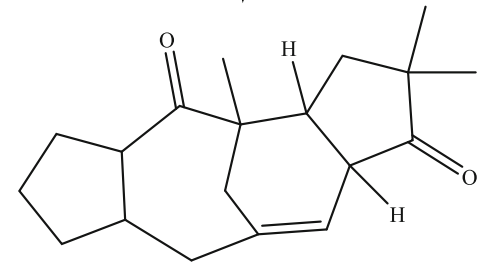

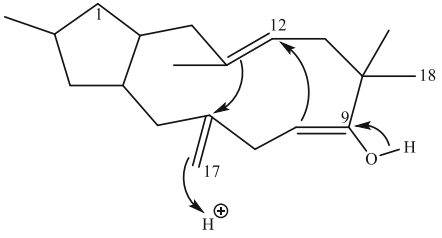

Jatropha-6(17), 12-diene

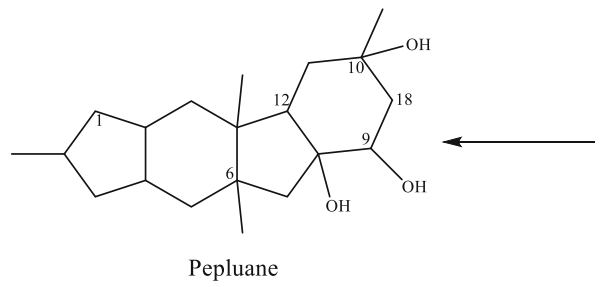

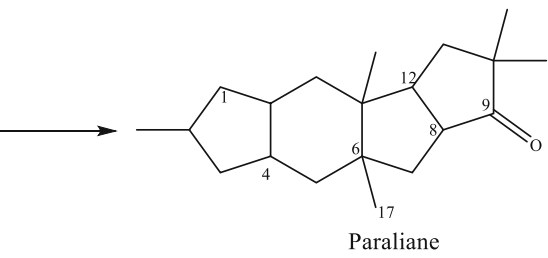

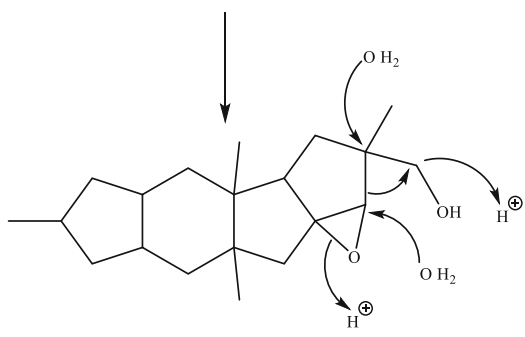

It is worth mentioning that the flexibility of the twelve-membered ring can adopt two main conformations: endo- and exo-type depending on the spatial orientation of the 6,17 exo-methylene group (Appendino et al. 1998; Jakupovic et al. 1998b, c; Marco et al. 1998). It is also reported that the conformational option depends on the acylation pattern on the jatrophane core (Corea et al. 2005a; Esposito et al. 2016; Günther et al. 1998). Diagnostic spectral features to discriminate between the two conformations are the ${ }^{3} J_{4,5}$ value and spatial close NOESY correlations (Appendino et al. 1998; Corea et al. 2005a; Jakupovic et al. 1998c). The large ${ }^{3} J_{4,5}=9-11 \mathrm{~Hz}$ coupling and the existence of a diagnostic NOESY cross-peak between $\mathrm{H}-5$ and $\mathrm{H}-17$, due to the perpendicular orientation of the exomethylene group to the mean plane of the macrocycle would advocate for a perpendicular endo-type conformation whereas small ${ }^{3} J_{4,5}=0-4 \mathrm{~Hz}$ coupling and interactions between $\mathrm{H}-4 / \mathrm{H}-7$ and $\mathrm{H}-5 / \mathrm{H}-8$ along with no interaction between $\mathrm{H}-5$ or $\mathrm{H}-7$ and the exomethylenic $\mathrm{H}-17$ could indicate a parallel exo-type 


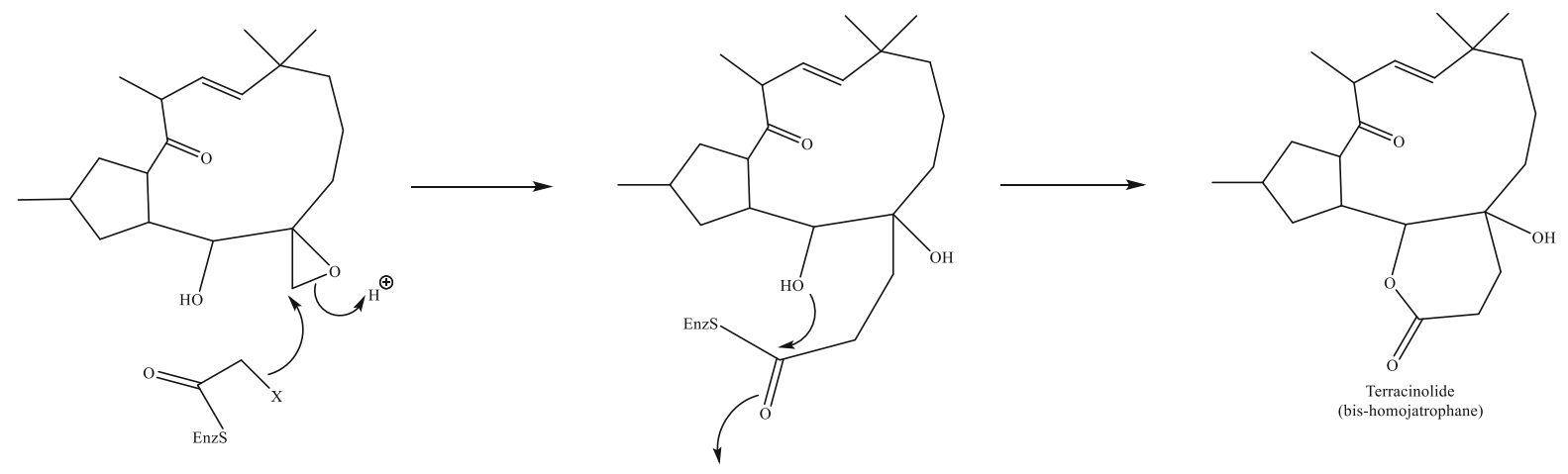

Fig. 12 Biogenesis of terracinolides by incorporation of a $C_{2}$ unit (from acetate or malonate) into jatrophane precursor

conformation (Appendino et al. 1998; Jakupovic et al. 1998a, b, c; Marco et al. 1998). For jatrophanes, conformational flexibility was reported to be important for P-gp modulation, since molecules with the macrocyclic jatrophane-type twelve-membered ring scaffold were generally found to be more active than the 5/8/8 fused ring systems like welwitschines $A$ and B. Similar observations were also found for rearranged polycyclic jatrophanes like segetane, paraliane and pepluane skeletons showing a lower P-gp modulatory efficiency when compared to molecules with the macrocyclic jatrophane-type scaffold (Ferreira et al. 2014; Reis et al. 2012).

\section{Isolation of diterpenes}

Diterpenes are generally isolated from various Euphorbia species by similar protocols. All parts of the plants may accumulate diterpenoids. The roots, leaves, stems, fruits, seeds and the whole plant are equally studied. Furthermore, Euphorbia plants are known to produce white irritant latex-containing different metabolites such as macrocyclic diterpenoids (Nothias-Scaglia et al. 2014, 2015a, c) and hence, it is commonly investigated as well (Fattorusso et al. 2002; Shi et al. 2008; Vasas and Hohmann 2014). In general, extraction of the plant materials performs at room temperature by maceration. The extracts are evaporated at reduced pressure at $40{ }^{\circ} \mathrm{C}$. Since the plants produce complex mixtures of structurally-related analogues whose core is the same and are differed from each other by the substitution pattern, then their isolation requires a multistep separation protocol. Mustafa Ghanadian and coworkers developed a five- step method for isolation and purification of macrocyclic diterpenes in nine Euphorbia species. The sample preparation includes: (A) the percolation or maceration of powdered plant material with $\mathrm{CH}_{2} \mathrm{Cl}_{2}$ :acetone (2:1) at room temperature, (B) extract is suspended in $\mathrm{MeOH}: \mathrm{H} 2 \mathrm{O}(75: 25)$ after concentration and subjected to vacuum filtration using a porcelain Buchner funnel with a vacuum pump and a large glass funnel filter with fritted sintered glass disc containing RP-18 adsorbent or silica gel pregnated with paraffin (15\%), eluting with $\mathrm{MeOH}: \mathrm{H} 2 \mathrm{O}(75: 25)$ as solvent, (C) the defatted fraction which is rich in diterpenoids and free from dark green chlorophylls and fats, is concentrated and loaded on the gravity silica gel column using mixtures comprising hexane: EtOAc of increasing polarity, D) resultant fractions being rich in macrocyclic diterpenoids are selected based on primary ${ }^{1} \mathrm{H}-\mathrm{NMR}$ analysis and are subjected to Sephadex LH-20 eluting by hexane:acetone:MeOH (30:10:60) to remove remaining chlorophyll and unwanted materials and to gain crude diterpenoidal subfraction. The concentrated fractions are screened by TLC using hexane:acetone (6:4) and (7:3) as mobile phases. TLCs are Sprayed by concentrated serium sulphate $1 \%$ in sulfuric acid $10 \%$ followed by heating at $105{ }^{\circ} \mathrm{C}$ for the visualization of the polyester diterpene spots visulalized in dark brown color spots with $\mathrm{Rf}$ values of 0.2-0.7. E) Fractions which are rich in diterpenes are subjected on silica prep HPLC column $(20 \times 250 \mathrm{~mm}, 5 \mu \mathrm{m})$ using hexane:EtOAc in stepwise gradient solvent system (90:10; 85:15; 80:20; $75: 25 ; 70: 30$ ) as final purification (Ayatollahi et al. 2010a, b, Ghanadian et al. 2013, 2015; Zolfaghari et al. 2016). 
Table 1 Isolated jatrophane diterpenoids

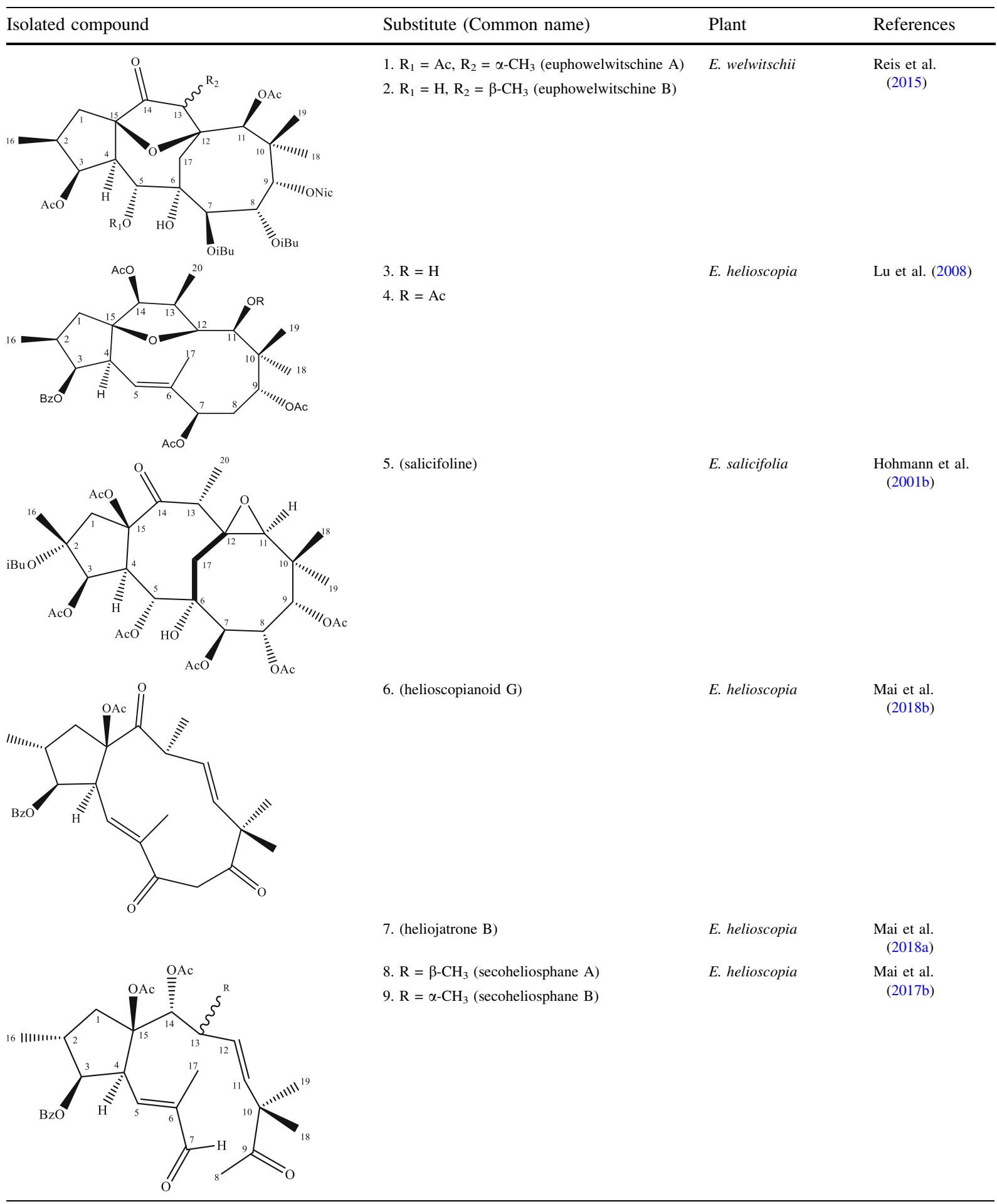


Table 1 continued

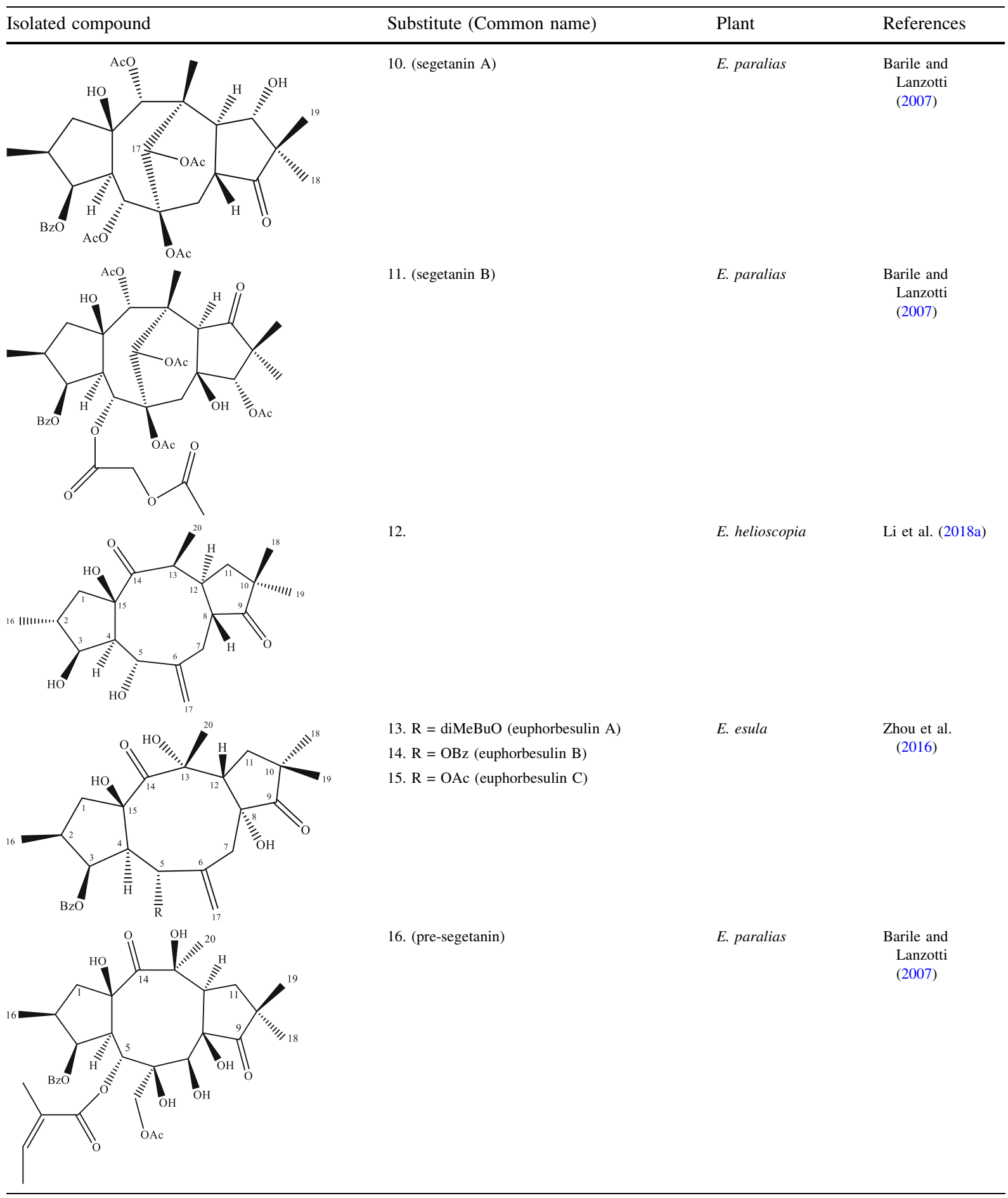


Table 1 continued

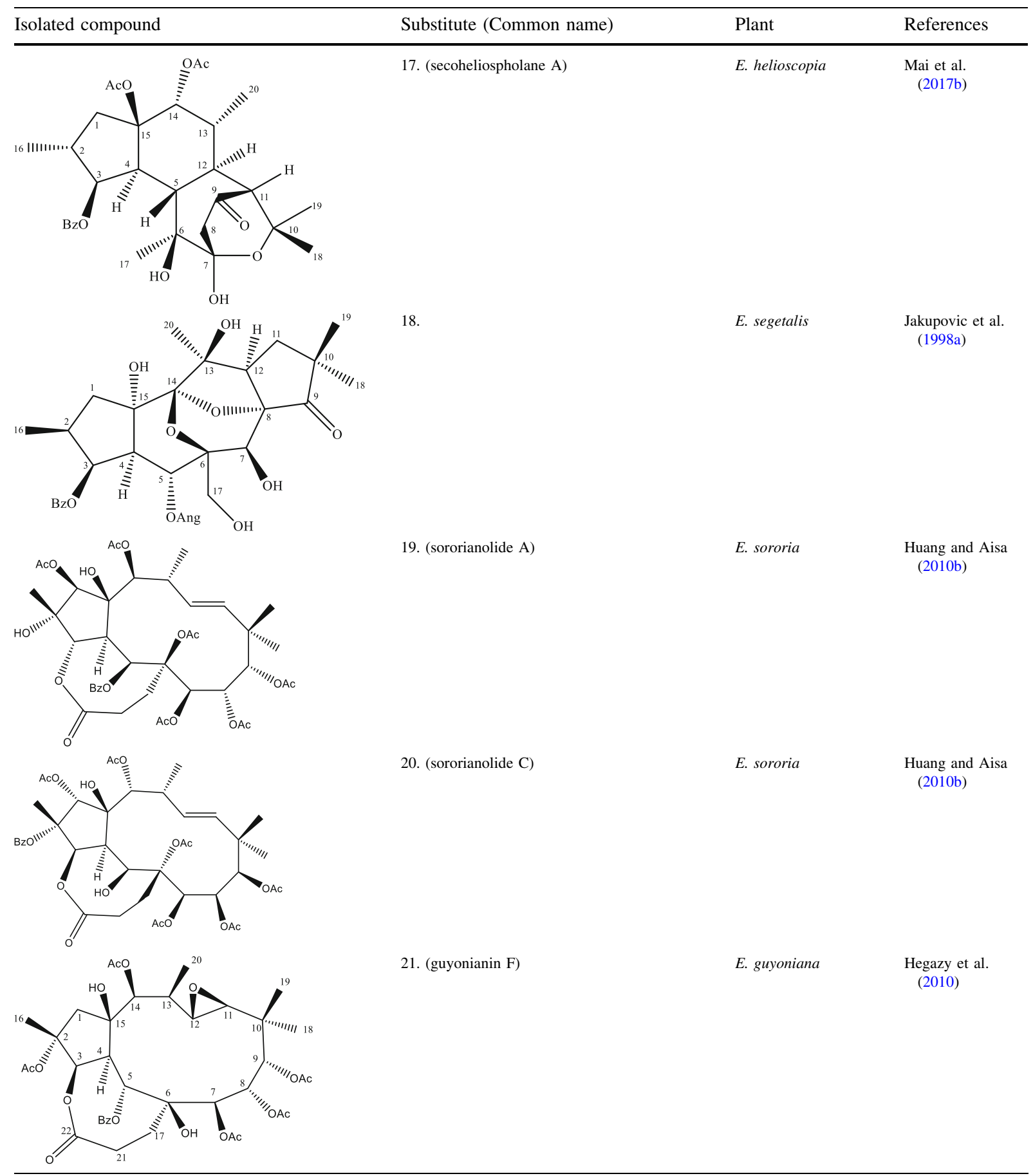


Table 1 continued

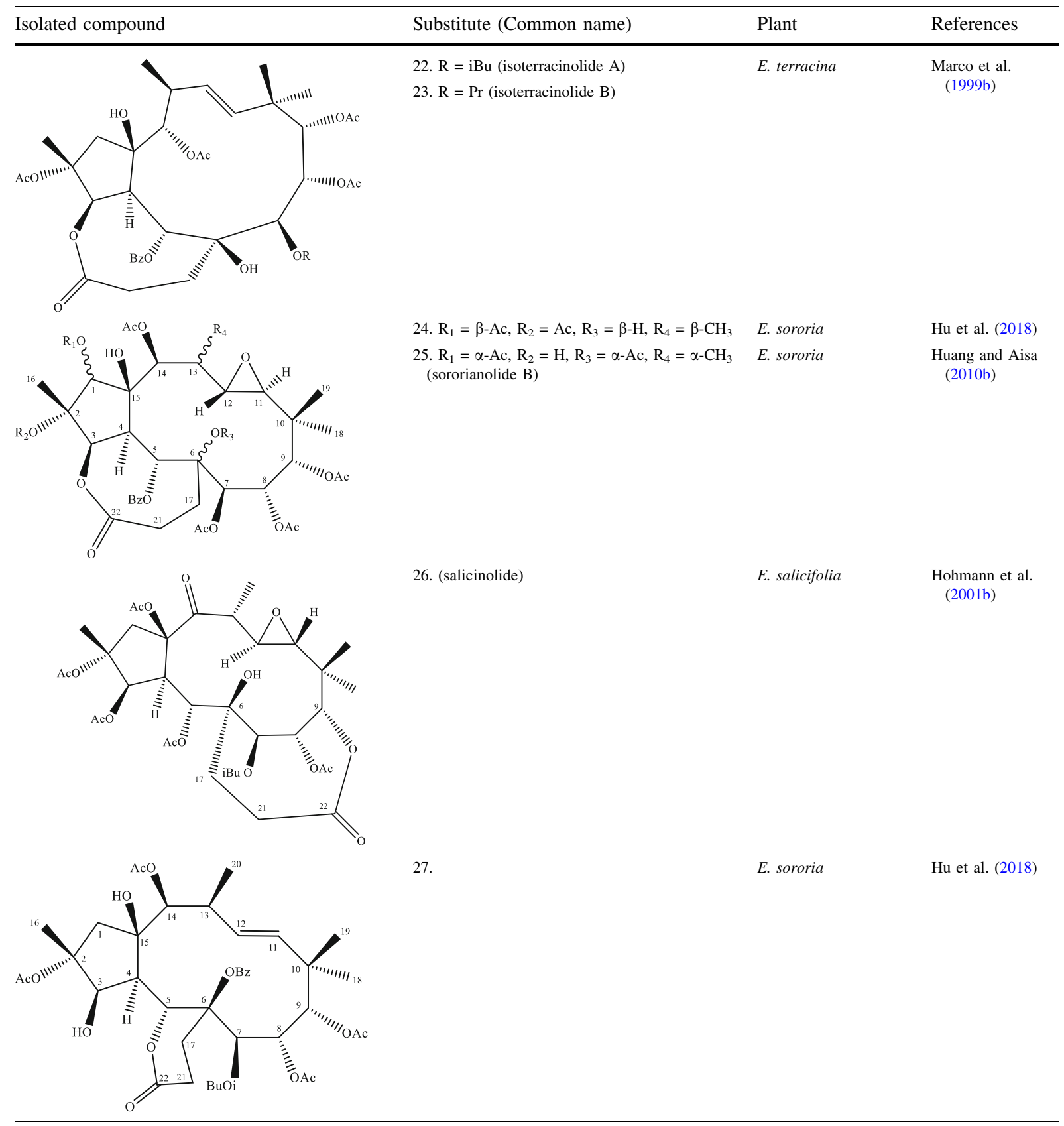


Table 1 continued

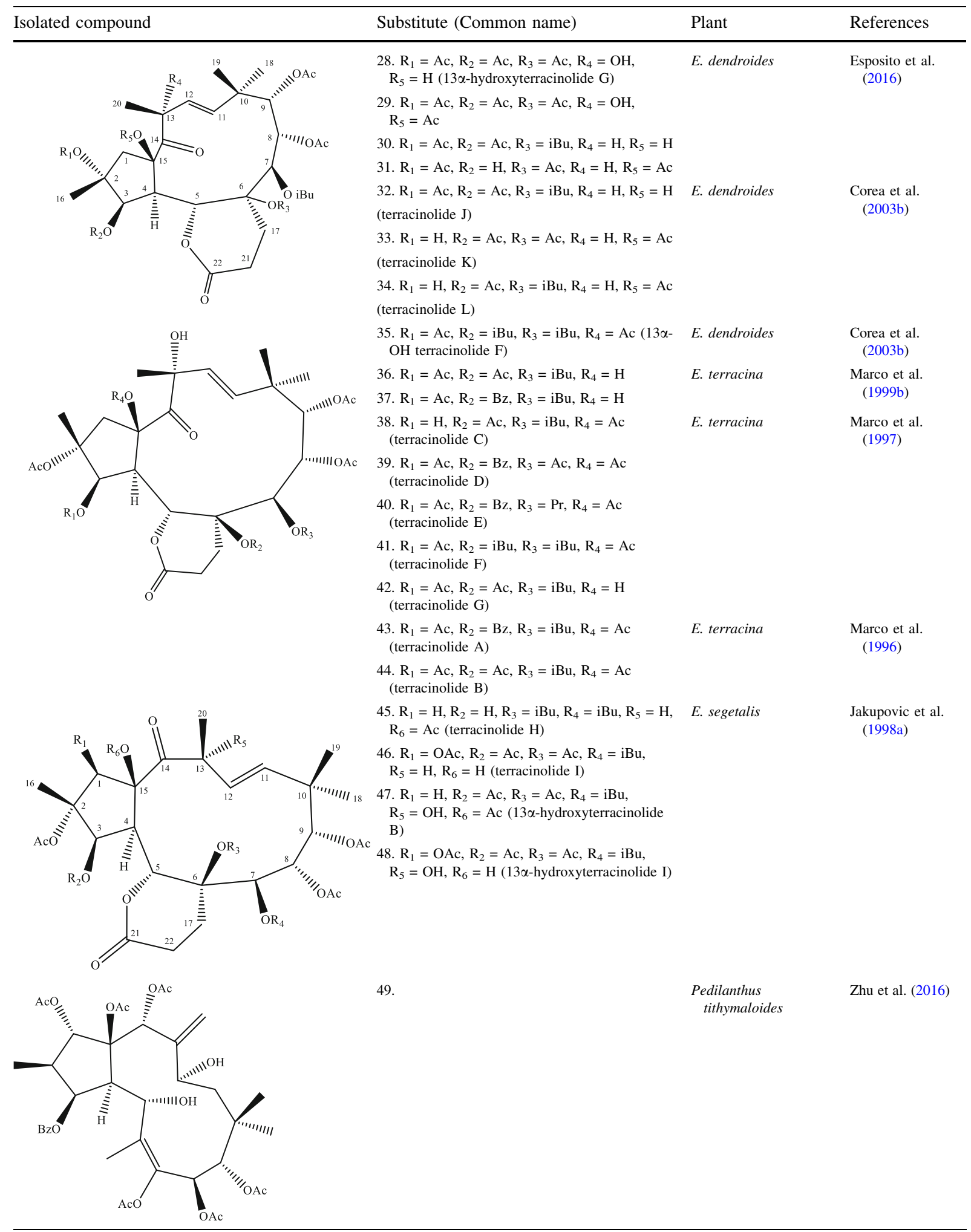


Table 1 continued

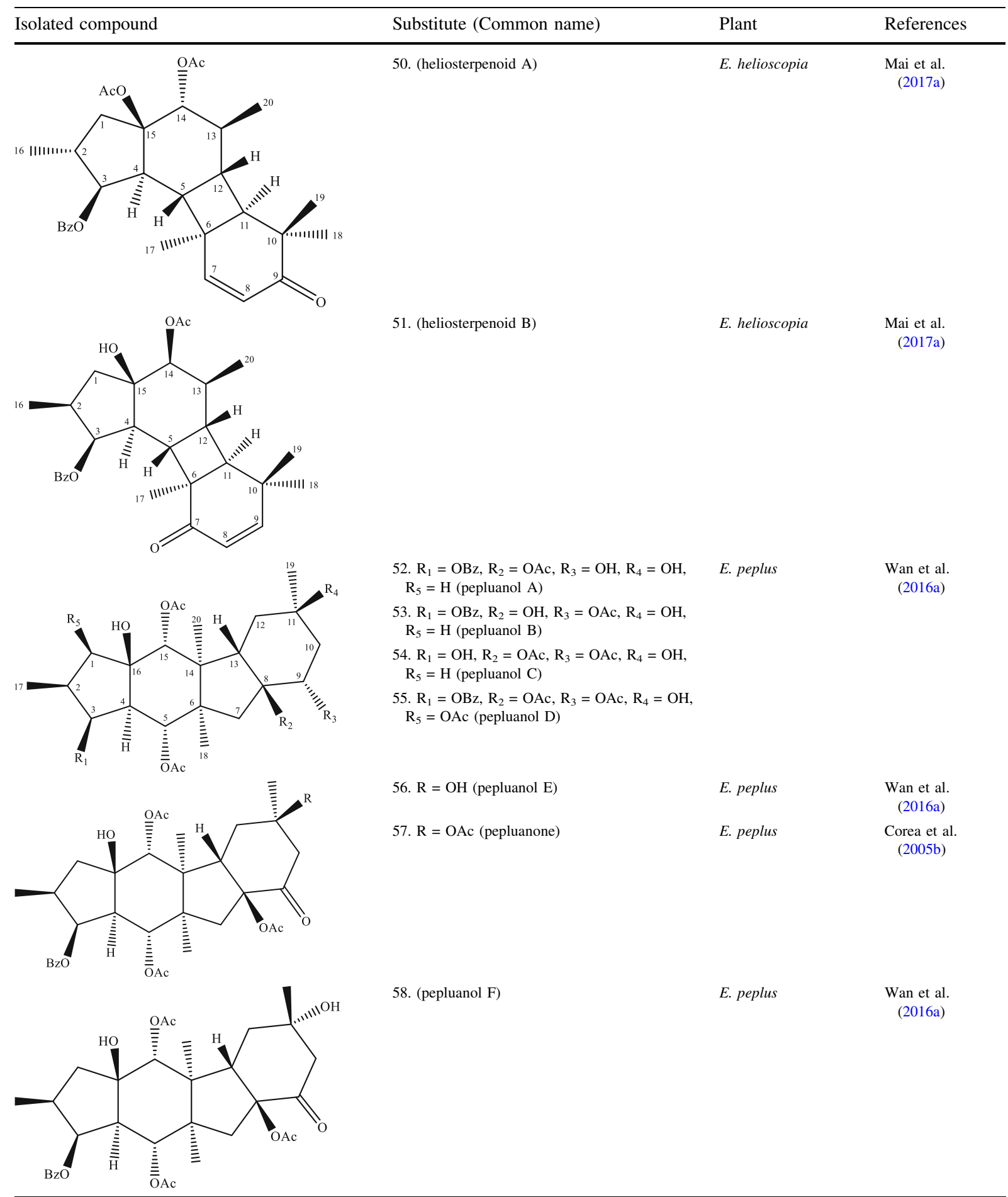


Table 1 continued

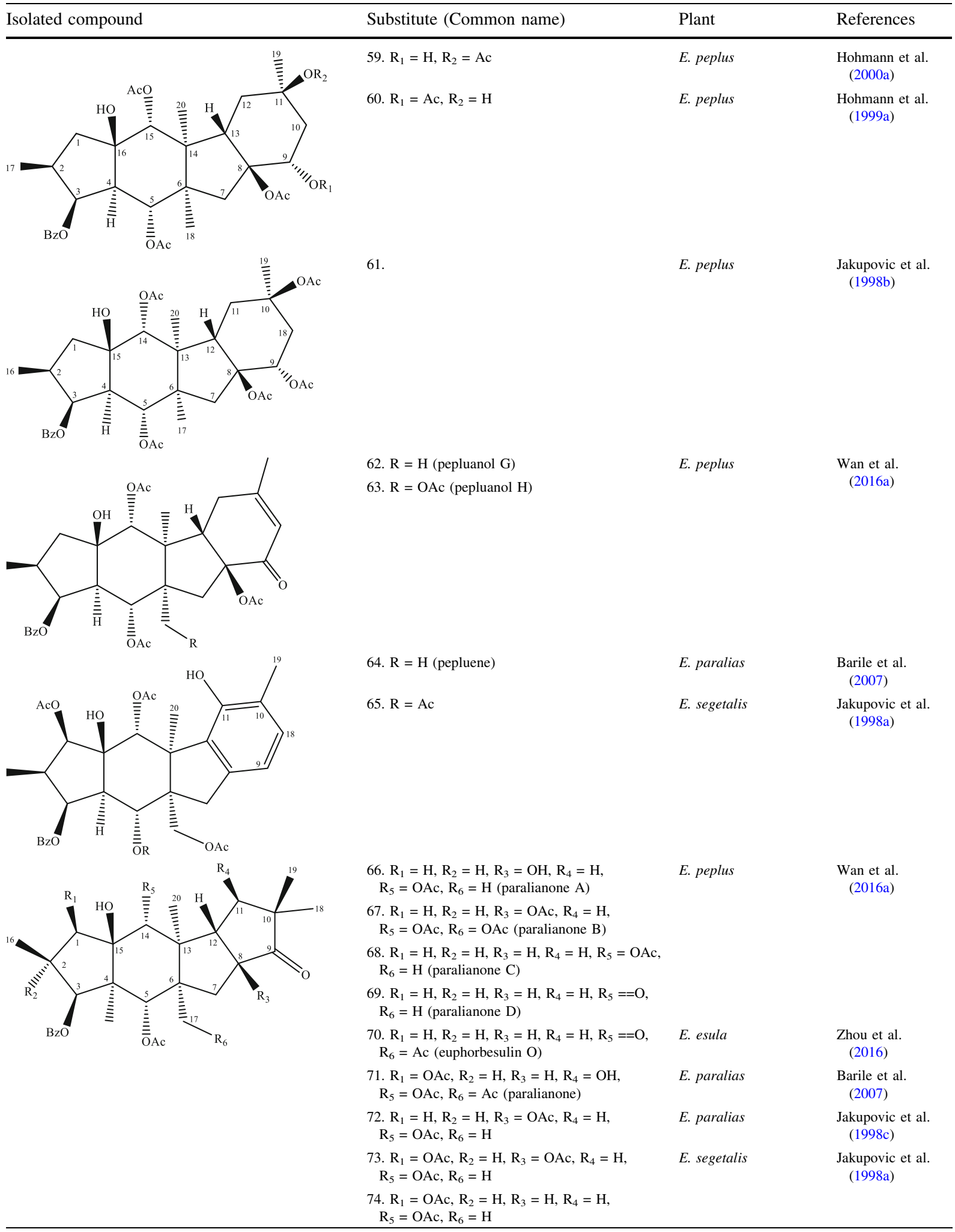


Table 1 continued

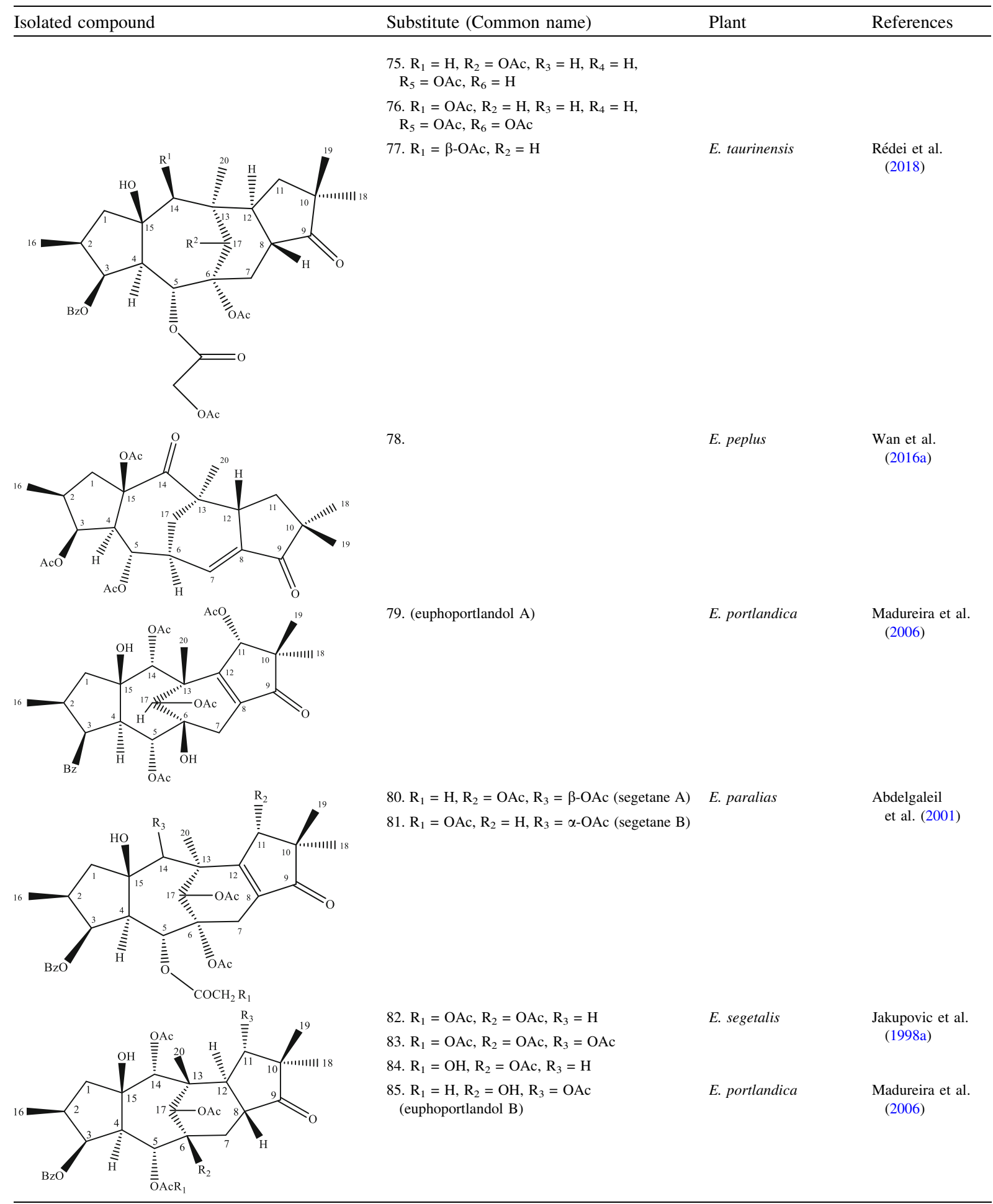


Table 1 continued

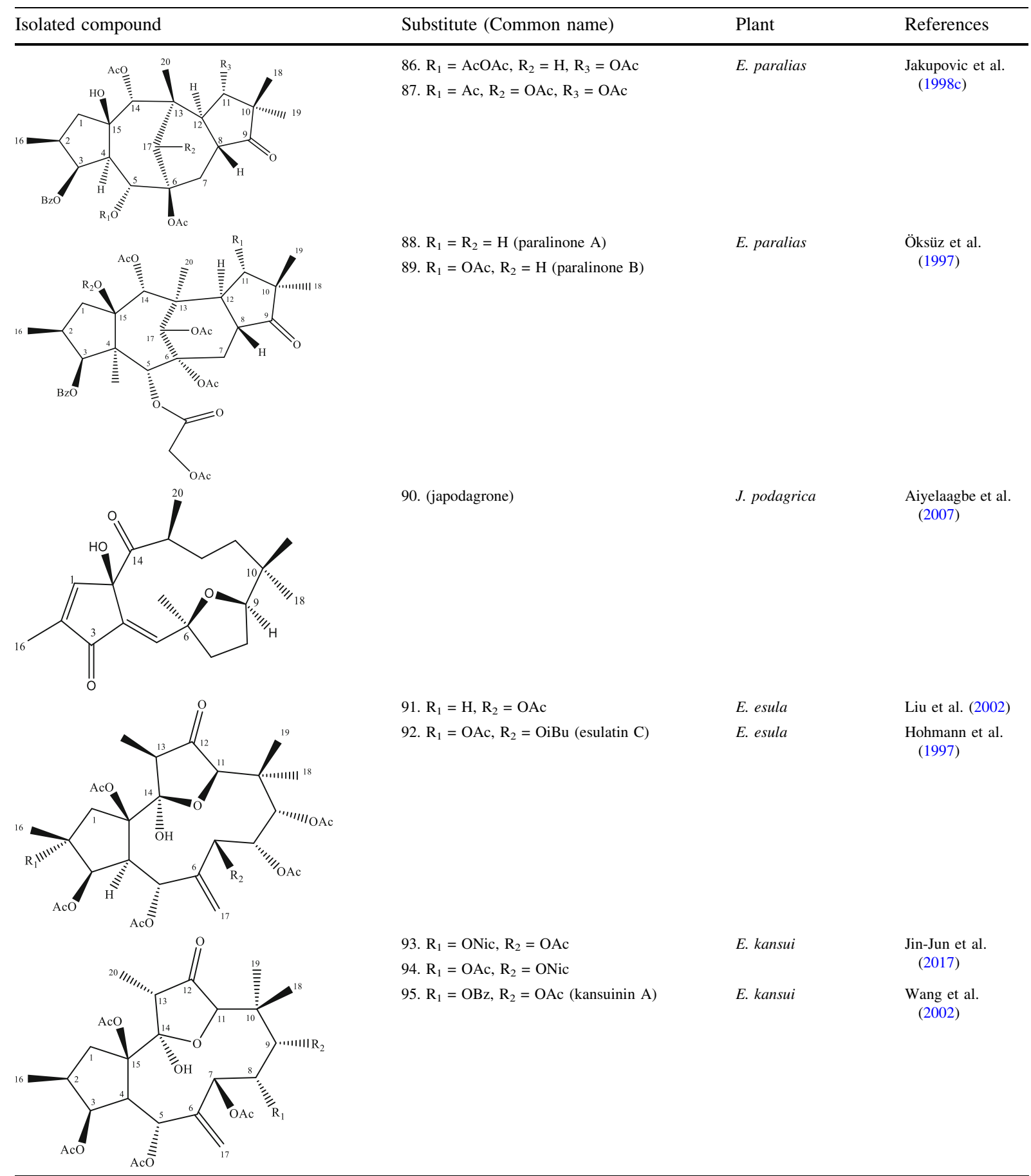


Table 1 continued

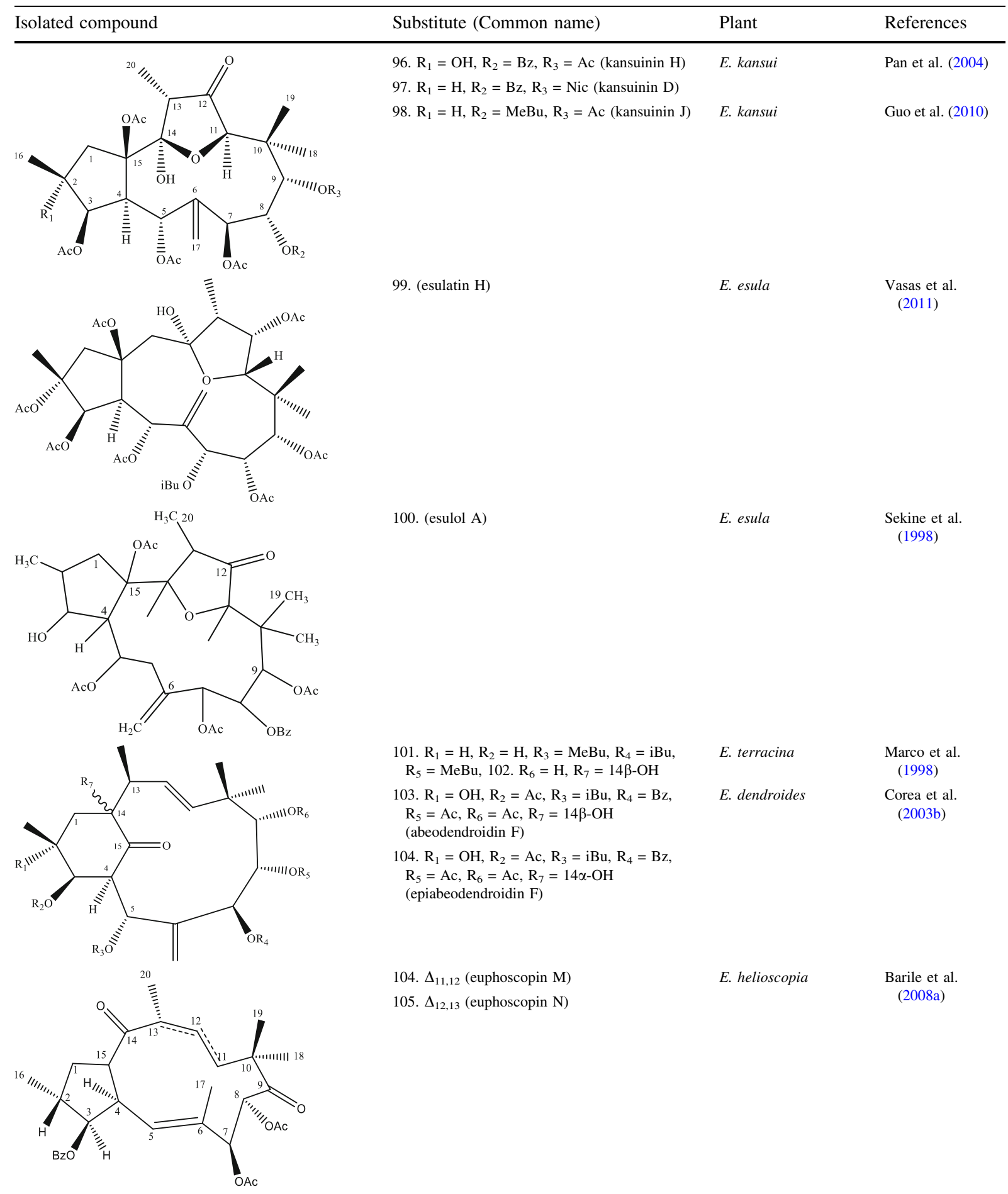


Table 1 continued

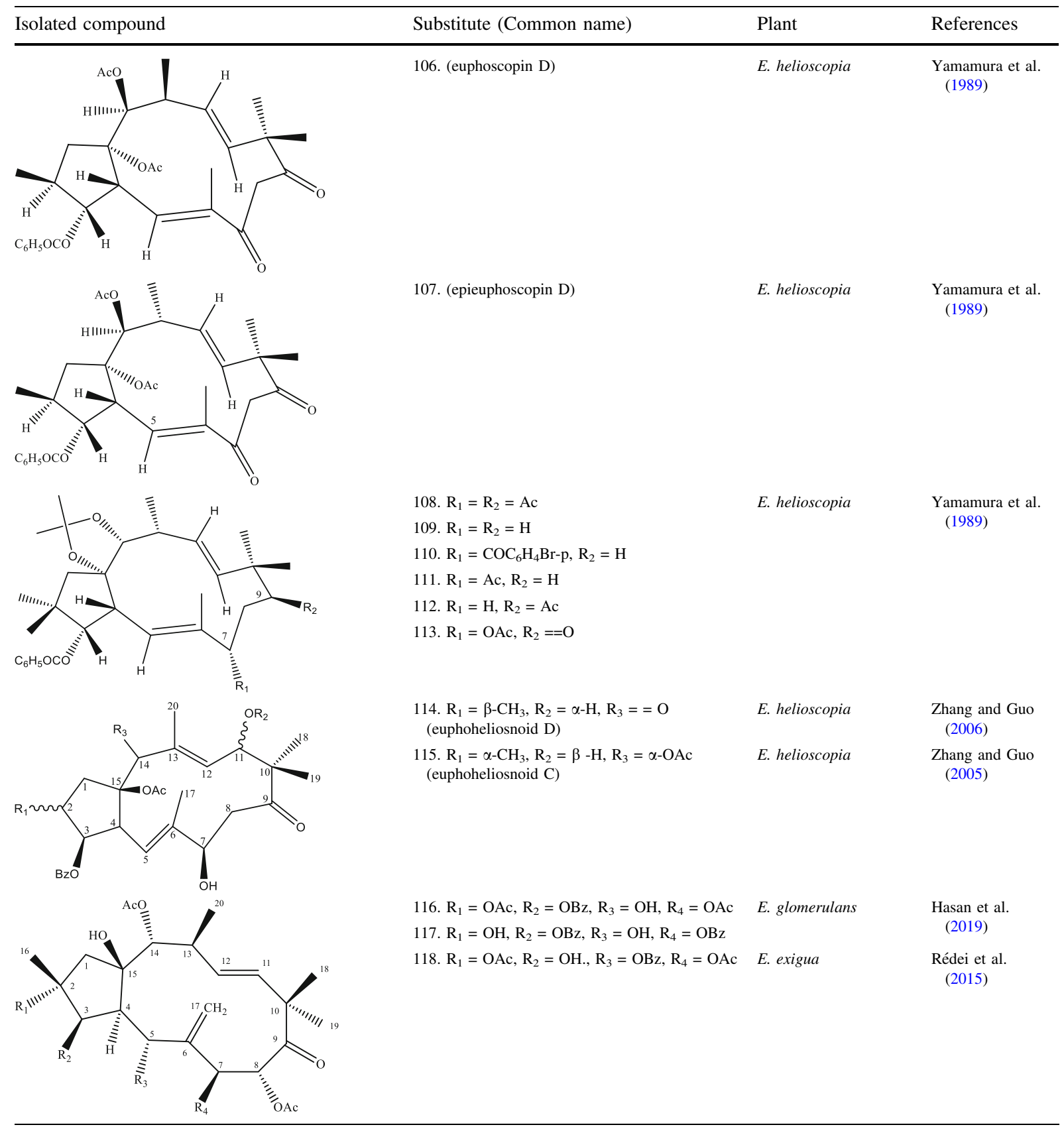


Table 1 continued

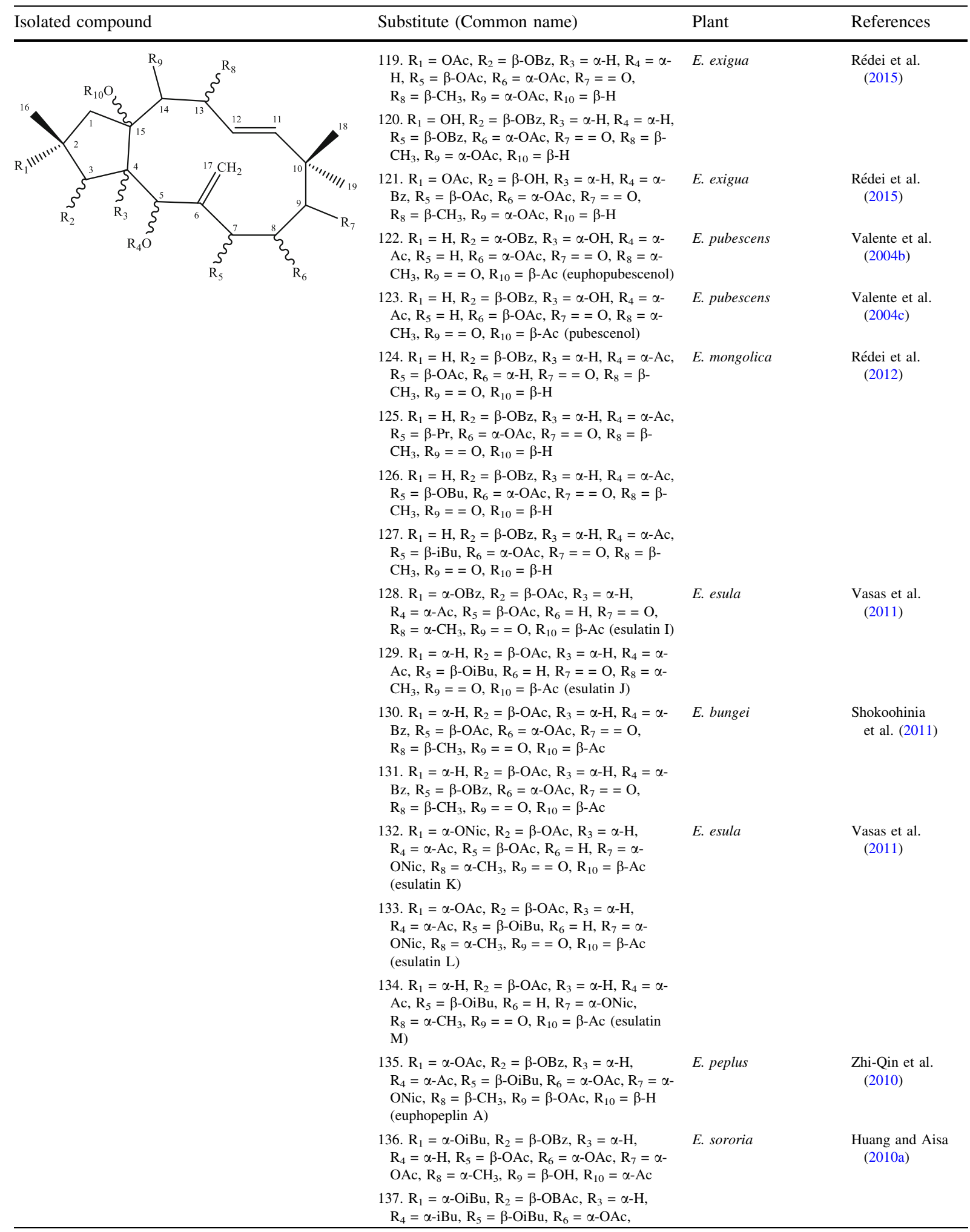


Table 1 continued

\begin{tabular}{|c|c|c|c|}
\hline Isolated compound & Substitute (Common name) & Plant & References \\
\hline \multicolumn{4}{|c|}{$\begin{array}{l}\mathrm{R}_{7}=\alpha-\mathrm{OAc}, \mathrm{R}_{8}=\alpha-\mathrm{CH}_{3}, \mathrm{R}_{9}=\beta-\mathrm{OBz} \\
\mathrm{R}_{10}=\alpha-\mathrm{H}\end{array}$} \\
\hline & $\begin{array}{l}\text { 138. } \mathrm{R}_{1}=\alpha-\mathrm{OiBu}, \mathrm{R}_{2}=\alpha-\mathrm{ONic}, \mathrm{R}_{3}=\beta-\mathrm{H}, \\
\mathrm{R}_{4}=\beta-\mathrm{Ac}, \mathrm{R}_{5}=\alpha-\mathrm{OAc}, \mathrm{R}_{6}=\alpha-\mathrm{OAc}, \mathrm{R}_{7}=\alpha- \\
\mathrm{OAc}, \mathrm{R}_{8}=\alpha-\mathrm{CH}_{3}, \mathrm{R}_{9}==\mathrm{O}, \mathrm{R}_{10}=\beta-\mathrm{Ac}\end{array}$ & \multirow[t]{4}{*}{ E. sororia } & \multirow[t]{4}{*}{$\begin{array}{l}\text { Huang and Aisa } \\
\text { (2010a) }\end{array}$} \\
\hline & $\begin{array}{l}\text { 139. } \mathrm{R}_{1}=\alpha-\mathrm{OiBu}, \mathrm{R}_{2}=\alpha-\mathrm{ONic}, \mathrm{R}_{3}=\beta-\mathrm{H}, \\
\mathrm{R}_{4}=\beta-\mathrm{Ac}, \mathrm{R}_{5}=\alpha-\mathrm{OiBu}, \mathrm{R}_{6}=\alpha-\mathrm{OAc}, \mathrm{R}_{7}=\alpha- \\
\mathrm{OAc}, \mathrm{R}_{8}=\alpha-\mathrm{CH}_{3}, \mathrm{R}_{9}==\mathrm{O}, \mathrm{R}_{10}=\beta-\mathrm{Ac}\end{array}$ & & \\
\hline & $\begin{array}{l}\text { 140. } \mathrm{R}_{1}=\alpha-\mathrm{OiBu}, \mathrm{R}_{2}=\alpha-\mathrm{OBZ}, \mathrm{R}_{3}=\beta-\mathrm{H} \\
\mathrm{R}_{4}=\beta-\mathrm{Ac}, \mathrm{R}_{5}=\alpha-\mathrm{OiBu}, \mathrm{R}_{6}=\alpha-\mathrm{OAc}, \mathrm{R}_{7}=\alpha- \\
\text { OAc, } \mathrm{R}_{8}=\alpha-\mathrm{CH}_{3}, \mathrm{R}_{9}==\mathrm{O}, \mathrm{R}_{10}=\beta-\mathrm{Ac}\end{array}$ & & \\
\hline & $\begin{array}{l}\text { 141. } \mathrm{R}_{1}=\alpha-\mathrm{OiBu}, \mathrm{R}_{2}=\alpha-\mathrm{ONic}, \mathrm{R}_{3}=\beta-\mathrm{H}, \\
\mathrm{R}_{4}=\beta-\mathrm{Ac}, \mathrm{R}_{5}=\alpha-\mathrm{OAc}, \mathrm{R}_{6}=\alpha-\mathrm{OAc}, \mathrm{R}_{7}=\alpha- \\
\mathrm{OAc}, \mathrm{R}_{8}=\alpha-\mathrm{CH}_{3}, \mathrm{R}_{9}==\mathrm{O}, \mathrm{R}_{10}=\beta-\mathrm{Ac}\end{array}$ & & \\
\hline & $\begin{array}{l}\text { 142. } \mathrm{R}_{1}=\alpha-\mathrm{OBz}, \mathrm{R}_{2}=\beta-\mathrm{OAc}, \mathrm{R}_{3}=\alpha-\mathrm{H}, \\
\mathrm{R}_{4}=\alpha-\mathrm{Ac}, \mathrm{R}_{5}=\beta-\mathrm{OBz}, \mathrm{R}_{6}=\alpha-\mathrm{OAc}, \mathrm{R}_{7}=\alpha- \\
\mathrm{OAc}, \mathrm{R}_{8}=\beta-\mathrm{CH}_{3}, \mathrm{R}_{9}==\mathrm{O}, \mathrm{R}_{10}=\beta-\mathrm{Ac} \\
\text { (euphotuckeyanol) }\end{array}$ & E. tuckeyana & $\begin{array}{l}\text { Duarte et al. } \\
\text { (2008) }\end{array}$ \\
\hline & $\begin{array}{l}\text { 143. } \mathrm{R}_{1}=\alpha-\mathrm{H}, \mathrm{R}_{2}=\beta-\mathrm{OAc}, \mathrm{R}_{3}=\alpha-\mathrm{H}, \mathrm{R}_{4}=\alpha- \\
\text { Ac, } \mathrm{R}_{5}=\beta-\mathrm{OMeBu}, \mathrm{R}_{6}=\alpha-\mathrm{OAc}, \mathrm{R}_{7}==\mathrm{O} \\
\mathrm{R}_{8}=\beta-\mathrm{CH}_{3}, \mathrm{R}_{9}=\beta-\mathrm{OBz}, \mathrm{R}_{10}=\beta-\mathrm{H} \\
\text { (tuckeyanol A) }\end{array}$ & \multirow[t]{2}{*}{ E. tuckeyana } & \multirow[t]{2}{*}{$\begin{array}{l}\text { Duarte et al. } \\
\text { (2008) }\end{array}$} \\
\hline & $\begin{array}{l}\text { 144. } \mathrm{R}_{1}=\alpha-\mathrm{H}, \mathrm{R}_{2}=\beta-\mathrm{OAc}, \mathrm{R}_{3}=\alpha-\mathrm{H}, \mathrm{R}_{4}=\alpha- \\
\mathrm{Ac}, \mathrm{R}_{5}=\beta-\mathrm{OiBu}, \mathrm{R}_{6}=\alpha-\mathrm{OAc}, \mathrm{R}_{7}==\mathrm{O} \\
\mathrm{R}_{8}=\beta-\mathrm{CH}_{3}, \mathrm{R}_{9}=\beta-\mathrm{OBz}, \mathrm{R}_{10}=\beta-\mathrm{H} \\
\text { (tuckeyanol B) }\end{array}$ & & \\
\hline & $\begin{array}{l}\text { 145. } \mathrm{R}_{1}=\alpha-\mathrm{ONic}, \mathrm{R}_{2}=\beta-\mathrm{OAc}, \mathrm{R}_{3}=\alpha-\mathrm{H}, \\
\mathrm{R}_{4}=\alpha-\mathrm{Bz}, \mathrm{R}_{5}=\beta-\mathrm{OAc}, \mathrm{R}_{6}=\alpha-\mathrm{OAc}, \mathrm{R}_{7}=\alpha- \\
\mathrm{OAc}, \mathrm{R}_{8}=\beta-\mathrm{CH}_{3}, \mathrm{R}_{9}==\mathrm{O}, \mathrm{R}_{10}=\beta-\mathrm{Ac} \\
\text { (guyonianin C) }\end{array}$ & \multirow[t]{2}{*}{ E. guyoniana } & \multirow[t]{2}{*}{$\begin{array}{l}\text { El-Bassuony } \\
\quad(2007)\end{array}$} \\
\hline & $\begin{array}{l}\text { 146. } \mathrm{R}_{1}=\alpha-\mathrm{H}, \mathrm{R}_{2}=\beta-\mathrm{OBz}, \mathrm{R}_{3}=\alpha-\mathrm{H}, \mathrm{R}_{4}=\alpha- \\
\mathrm{Ac}, \mathrm{R}_{5}=\mathrm{H}, \mathrm{R}_{6}=\mathrm{H}, \mathrm{R}_{7}=\alpha-\mathrm{OAc}, \mathrm{R}_{8}=\beta-\mathrm{CH}_{3} \text {, } \\
\mathrm{R}_{9}==\mathrm{O}, \mathrm{R}_{10}=\beta-\mathrm{Ac} \text { (guyonianin } \mathrm{D} \text { ) }\end{array}$ & & \\
\hline & $\begin{array}{l}\text { 147. } \mathrm{R}_{1}=\alpha-\mathrm{ONic}, \mathrm{R}_{2}=\beta-\mathrm{OAc}, \mathrm{R}_{3}=\alpha-\mathrm{H} \\
\mathrm{R}_{4}=\alpha-\mathrm{Bz}, \mathrm{R}_{5}=\beta-\mathrm{OAc}, \mathrm{R}_{6}=\alpha-\mathrm{OAc}, \mathrm{R}_{7}=\alpha- \\
\mathrm{OAc}, \mathrm{R}_{8}=\beta-\mathrm{CH}_{3}, \mathrm{R}_{9}==\mathrm{O}, \mathrm{R}_{10}=\beta-\mathrm{H} \\
\text { (guyonianin A) }\end{array}$ & \multirow[t]{2}{*}{ E. guyoniana } & \multirow[t]{2}{*}{$\begin{array}{l}\text { Ahmed et al. } \\
\text { (2006) }\end{array}$} \\
\hline & $\begin{array}{l}\text { 148. } \mathrm{R}_{1}=\alpha-\mathrm{H}, \mathrm{R}_{2}=\beta-\mathrm{OBz}, \mathrm{R}_{3}=\alpha-\mathrm{H}, \mathrm{R}_{4}=\alpha- \\
\mathrm{Ac}, \mathrm{R}_{5}=\mathrm{H}, \mathrm{R}_{6}=\mathrm{H}, \mathrm{R}_{7}==\mathrm{O}, \mathrm{R}_{8}=\beta-\mathrm{CH}_{3} \\
\mathrm{R}_{9}==\mathrm{O}, \mathrm{R}_{10}=\beta-\mathrm{Ac}(\text { guyonianin } \mathrm{B})\end{array}$ & & \\
\hline & $\begin{array}{l}\text { 149. } \mathrm{R}_{1}=\alpha-\mathrm{OAc}, \mathrm{R}_{2}=\beta-\mathrm{OAc}, \mathrm{R}_{3}=\alpha-\mathrm{H} \\
\mathrm{R}_{4}=\alpha-\mathrm{MeBu}, \mathrm{R}_{5}=\beta-\mathrm{OiBu}, \mathrm{R}_{6}=\alpha-\mathrm{OAc} \\
\mathrm{R}_{7}=\alpha-\mathrm{OAc}, \mathrm{R}_{8}=\beta-\mathrm{CH}_{3}, \mathrm{R}_{9}=\beta-\mathrm{OBz} \\
\mathrm{R}_{10}=\beta-\mathrm{H}\end{array}$ & \multirow[t]{2}{*}{ E. sororia } & \multirow[t]{2}{*}{ Hu et al. (2018) } \\
\hline & $\begin{array}{l}\text { 150. } \mathrm{R}_{1}=\alpha-\mathrm{OiBu}, \mathrm{R}_{2}=\beta-\mathrm{OAc}, \mathrm{R}_{3}=\alpha-\mathrm{H}, \\
\mathrm{R}_{4}=\alpha-\mathrm{Bz}, \mathrm{R}_{5}=\beta-\mathrm{OiBu}, \mathrm{R}_{6}=\alpha-\mathrm{OAc}, \mathrm{R}_{7}=\alpha- \\
\mathrm{OAc}, \mathrm{R}_{8}=\beta-\mathrm{CH}_{3}, \mathrm{R}_{9}=\beta-\mathrm{OH}, \mathrm{R}_{10}=\beta-\mathrm{H}\end{array}$ & & \\
\hline $\mathrm{R}_{7} \mathrm{O}$ & $\begin{array}{l}\text { 151. } \mathrm{R}_{1}=\beta-\mathrm{CH}_{3}, \mathrm{R}_{2}=\mathrm{H}, \mathrm{R}_{3}=\mathrm{Tig}, \mathrm{R}_{4}=\mathrm{H} \\
\mathrm{R}_{5}=\alpha-\mathrm{OAc}, \mathrm{R}_{6}=\beta-\mathrm{CH}_{3}, \mathrm{R}_{7}=\beta-\mathrm{Ac}, \mathrm{R}_{8}=\mathrm{H}\end{array}$ & \multirow[t]{7}{*}{ E. dulcis } & \multirow[t]{7}{*}{$\begin{array}{l}\text { Kusz et al. } \\
\text { (2018) }\end{array}$} \\
\hline & $\begin{array}{r}\text { 152. } \mathrm{R}_{1}=\beta-\mathrm{CH}_{3}, \mathrm{R}_{2}=\mathrm{H}, \mathrm{R}_{3}=\mathrm{Tig}, \mathrm{R}_{4}=\mathrm{OAc}, \\
\mathrm{R}_{5}=\alpha-\mathrm{OAc}, \mathrm{R}_{6}=\beta-\mathrm{CH}_{3}, \mathrm{R}_{7}=\beta-\mathrm{Ac}, \mathrm{R}_{8}=\mathrm{H}\end{array}$ & & \\
\hline & $\begin{array}{l}\text { 153. } \mathrm{R}_{1}=\beta-\mathrm{CH}_{3}, \mathrm{R}_{2}=\mathrm{H}, \mathrm{R}_{3}=\mathrm{Tig}, \mathrm{R}_{4}=\mathrm{OAc} \\
\mathrm{R}_{5}=\alpha-\mathrm{OAc}, \mathrm{R}_{6}=\beta-\mathrm{CH}_{3}, \mathrm{R}_{7}=\beta-\mathrm{Tig}, \mathrm{R}_{8}=\mathrm{H}\end{array}$ & & \\
\hline $\mathrm{BzO}$ & $\begin{array}{l}\text { 154. } \mathrm{R}_{1}=\beta-\mathrm{CH}_{3}, \mathrm{R}_{2}=\mathrm{H}, \mathrm{R}_{3}=\mathrm{H}, \mathrm{R}_{4}=\mathrm{OAc}, \\
\mathrm{R}_{5}=\alpha-\mathrm{OAc}, \mathrm{R}_{6}=\beta-\mathrm{CH}_{3}, \mathrm{R}_{7}=\beta-\mathrm{Tig}, \mathrm{R}_{8}=\mathrm{H}\end{array}$ & & \\
\hline & $\begin{array}{l}\text { 155. } \mathrm{R}_{1}=\beta-\mathrm{CH}_{3}, \mathrm{R}_{2}=\mathrm{H}, \mathrm{R}_{3}=\mathrm{H}, \mathrm{R}_{4}=\mathrm{OAc} \\
\mathrm{R}_{5}=\alpha-\mathrm{OAc}, \mathrm{R}_{6}=\beta-\mathrm{CH}_{3}, \mathrm{R}_{7}=\beta-\mathrm{Ac}, \mathrm{R}_{8}=\mathrm{H}\end{array}$ & & \\
\hline & $\begin{array}{l}\text { 156. } \mathrm{R}_{1}=\beta-\mathrm{CH}_{3}, \mathrm{R}_{2}=\mathrm{H}, \mathrm{R}_{3}=\mathrm{Ac}, \mathrm{R}_{4}=\mathrm{OH} \\
\mathrm{R}_{5}=\alpha-\mathrm{OAc}, \mathrm{R}_{6}=\beta-\mathrm{CH}_{3}, \mathrm{R}_{7}=\beta-\mathrm{Ac}, \mathrm{R}_{8}=\mathrm{H}\end{array}$ & & \\
\hline & $\begin{array}{l}\text { 157. } \mathrm{R}_{1}=\beta-\mathrm{CH}_{3}, \mathrm{R}_{2}=\mathrm{H}, \mathrm{R}_{3}=\mathrm{Ac}, \mathrm{R}_{4}=\mathrm{OH} \\
\mathrm{R}_{5}=\alpha-\mathrm{OH}, \mathrm{R}_{6}=\beta-\mathrm{CH}_{3}, \mathrm{R}_{7}=\beta-\mathrm{Ac}, \mathrm{R}_{8}=\mathrm{H}\end{array}$ & & \\
\hline
\end{tabular}


Table 1 continued

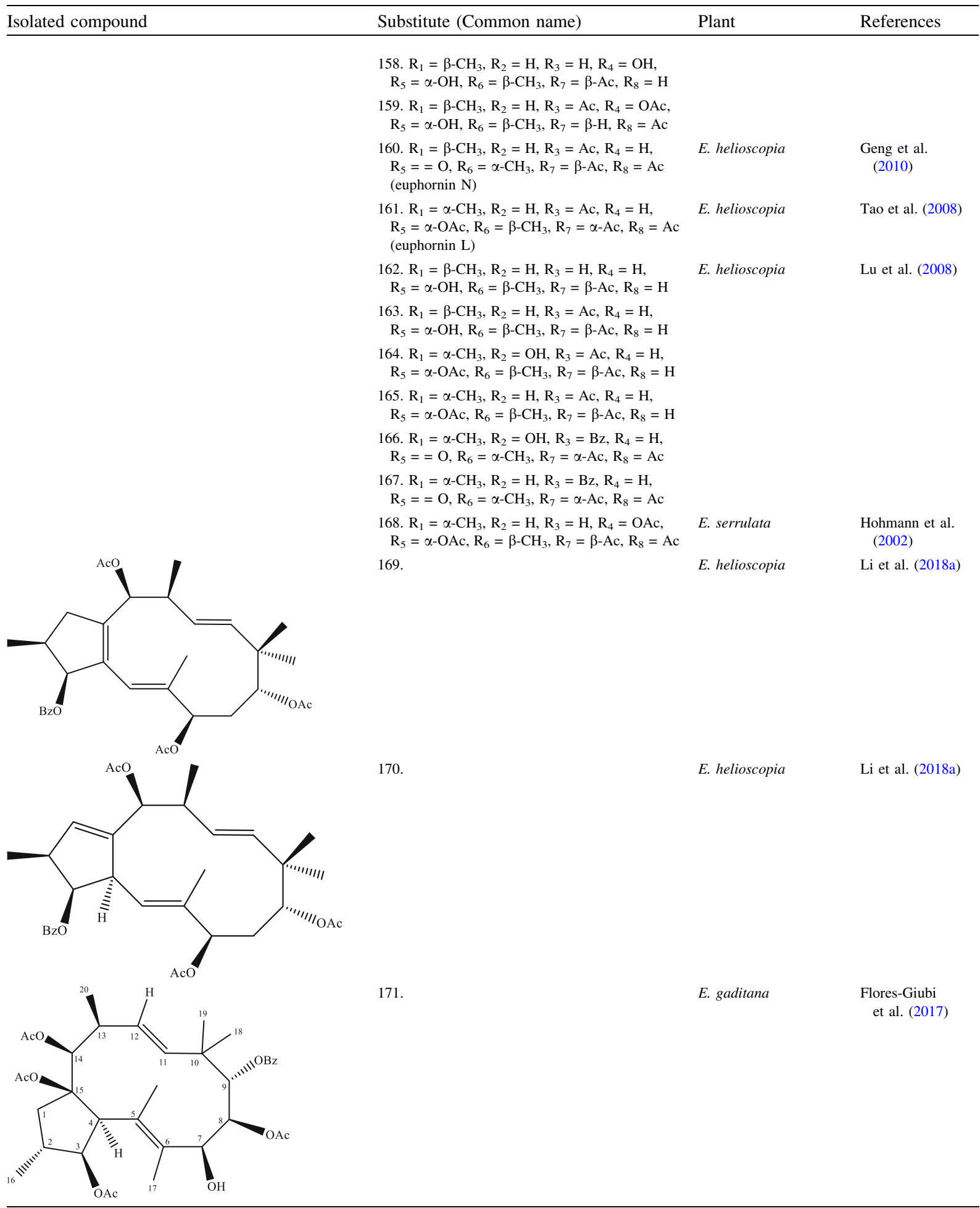


Table 1 continued

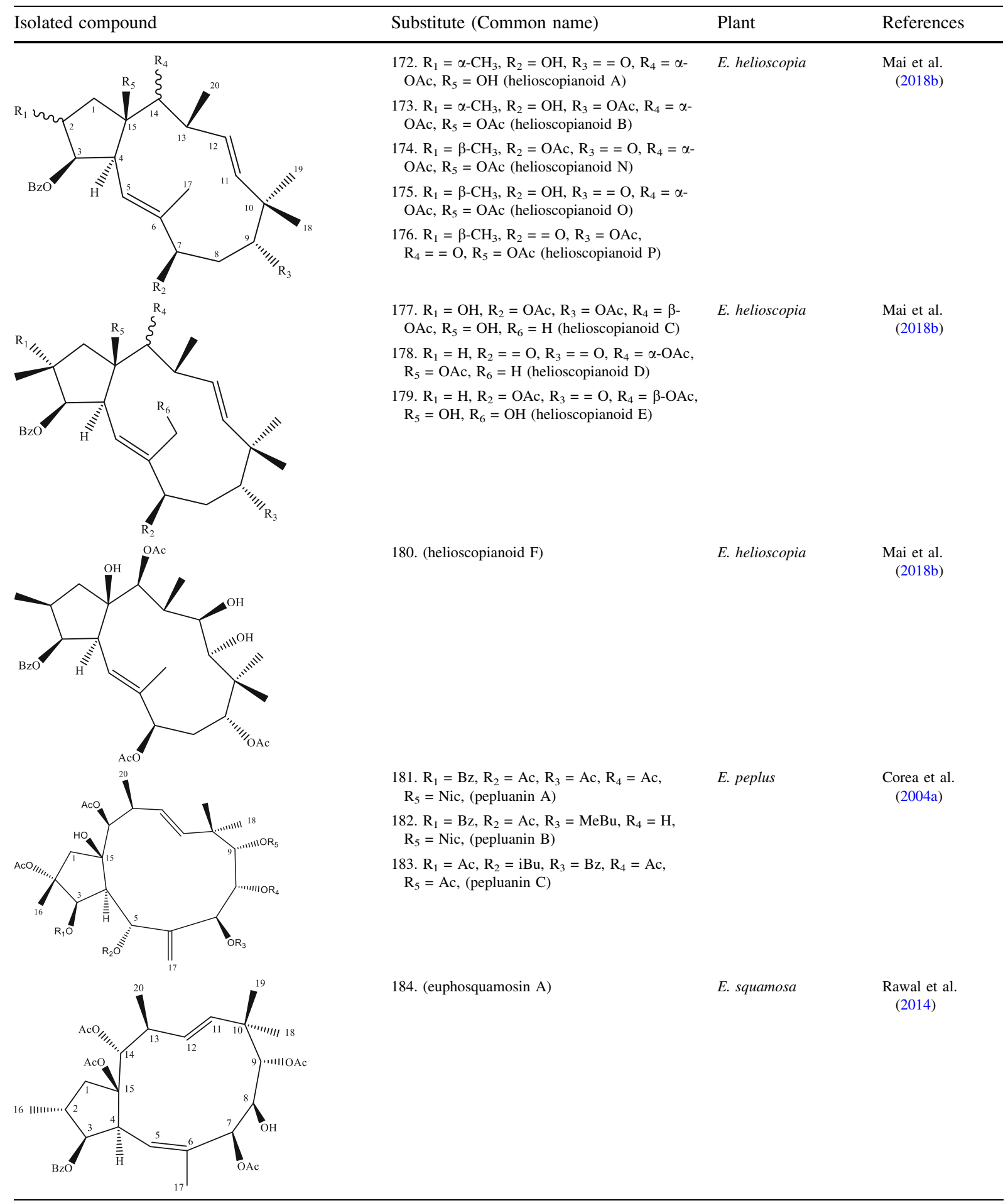


Table 1 continued

\begin{tabular}{|c|c|c|c|}
\hline Isolated compound & Substitute (Common name) & Plant & References \\
\hline & 185. (euphosquamosin B) & E. squamosa & $\begin{array}{l}\text { Rawal et al. } \\
\text { (2014) }\end{array}$ \\
\hline & $\begin{array}{l}\text { 186. } \mathrm{R}=\mathrm{Bz} \\
\text { 187. } \mathrm{R}=\mathrm{H}\end{array}$ & $\begin{array}{l}\text { Pedilanthus } \\
\quad \text { tithymaloides }\end{array}$ & $\begin{array}{l}\text { Mongkolvisut } \\
\text { and } \\
\text { Sutthivaiyakit } \\
(2007)\end{array}$ \\
\hline & 188. & $\begin{array}{l}\text { Pedilanthus } \\
\text { tithymaloides }\end{array}$ & $\begin{array}{l}\text { Mongkolvisut } \\
\text { and } \\
\text { Sutthivaiyakit } \\
\text { (2007) }\end{array}$ \\
\hline & $\begin{array}{l}\text { 189. } R=H \\
190 . R=A c\end{array}$ & $\begin{array}{l}\text { Pedilanthus } \\
\quad \text { tithymaloides }\end{array}$ & $\begin{array}{l}\text { Mongkolvisut } \\
\text { and } \\
\text { Sutthivaiyakit } \\
(2007)\end{array}$ \\
\hline & 191. & $\begin{array}{l}\text { Pedilanthus } \\
\quad \text { tithymaloides }\end{array}$ & $\begin{array}{l}\text { Mongkolvisut } \\
\text { and } \\
\text { Sutthivaiyakit } \\
\text { (2007) }\end{array}$ \\
\hline
\end{tabular}


Table 1 continued

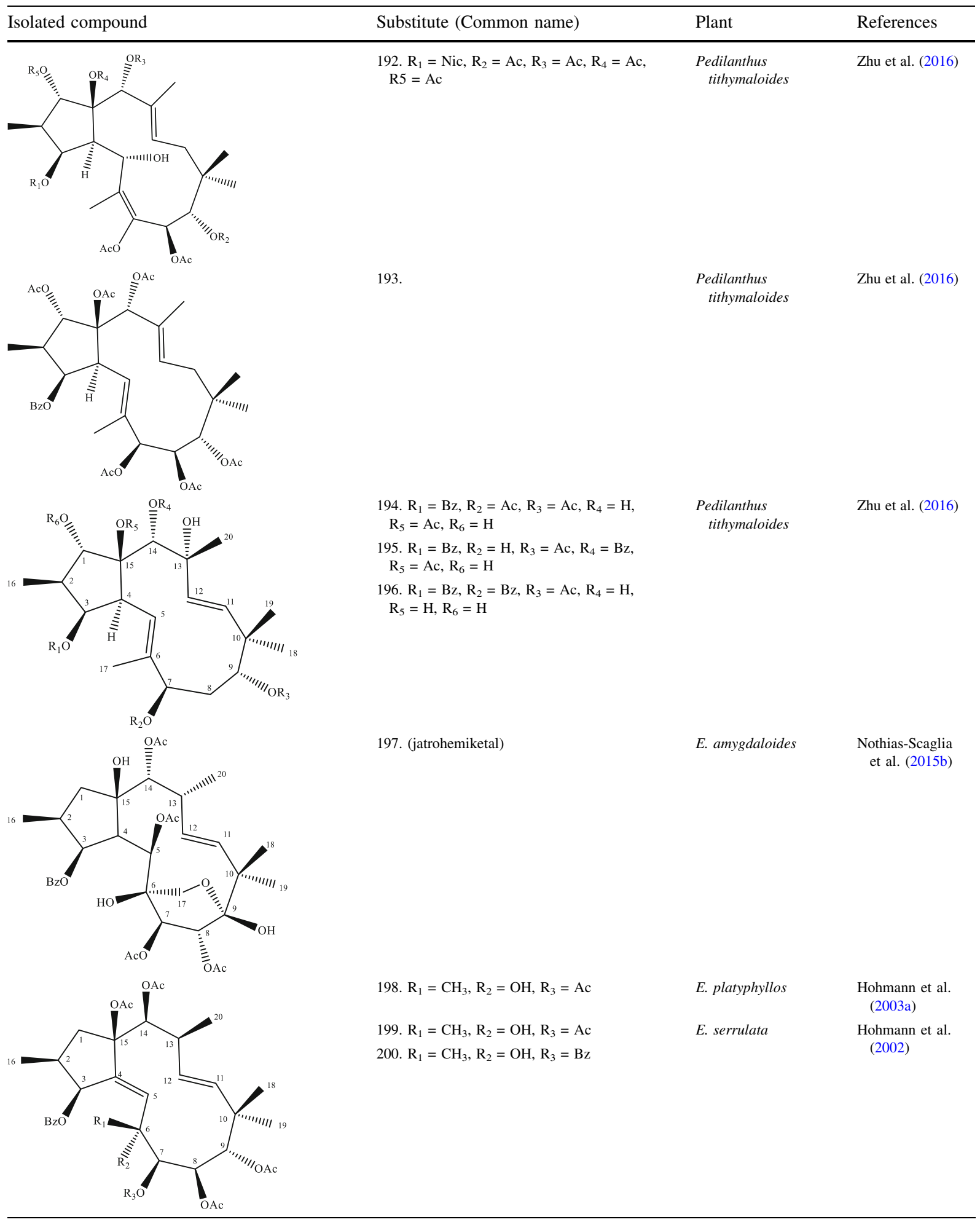


Table 1 continued

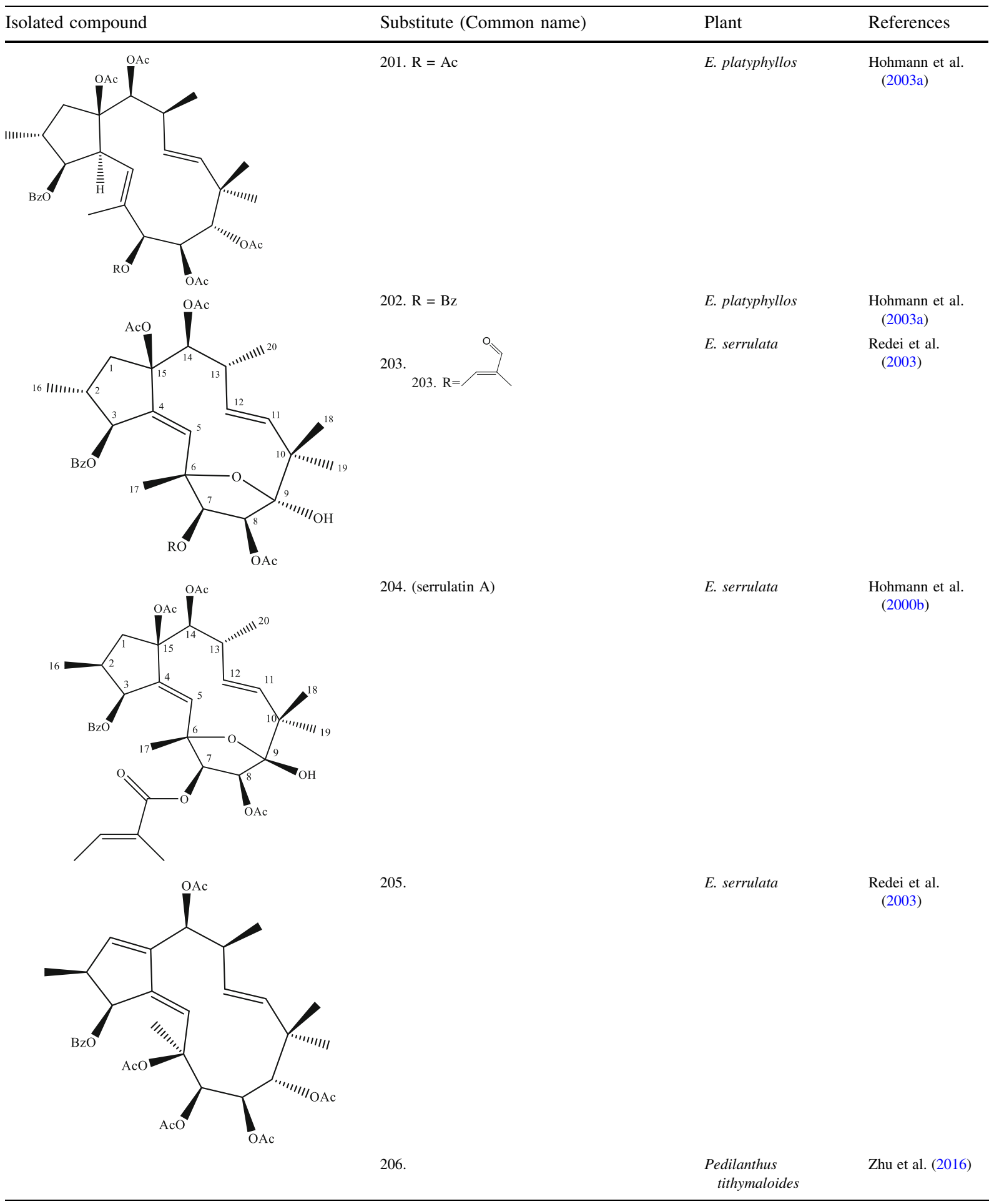


Table 1 continued

Isolated compound
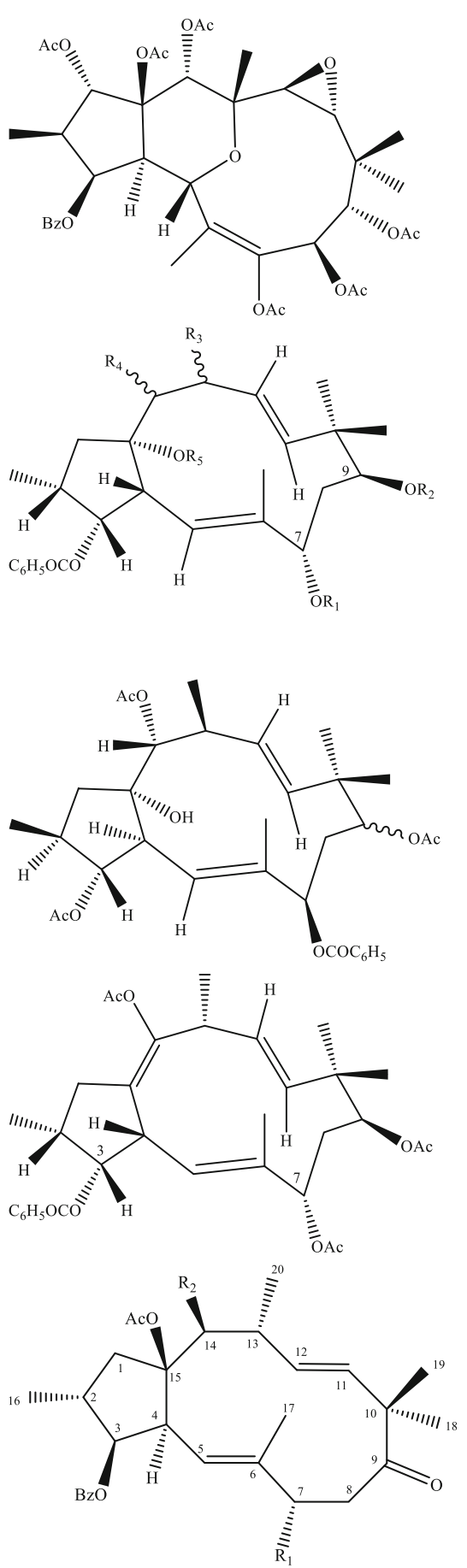

Substitute (Common name)

Plant

References

207. $R_{1}=H, R_{2}=H, R_{3}=\beta-C_{3}, R_{4}=\beta-O A c$, $\mathrm{R}_{5}=\mathrm{H}$

E. helioscopia

Yamamura et al. (1989)

208. $R_{1}=A c, R_{2}=A c, R_{3}=\alpha-C_{3}, R_{4}=\alpha-$

OAc, $\mathrm{R}_{5}=\mathrm{H}$ (euphornin)

209. $\mathrm{R}_{1}=\mathrm{H}, \mathrm{R}_{2}=\mathrm{H}, \mathrm{R}_{3}=\alpha-\mathrm{CH}_{3}, \mathrm{R}_{4}=\alpha-\mathrm{OH}$,

$\mathrm{R}_{5}=\mathrm{H}$

210. $\mathrm{R}_{1}=\mathrm{H}, \mathrm{R}_{2}=\mathrm{Ac}, \mathrm{R}_{3}=\alpha-\mathrm{CH}_{3}, \mathrm{R}_{4}=\alpha-\mathrm{OAc}$,

$\mathrm{R}_{5}=\mathrm{H}$ (euphornin A)

211. $\mathrm{R}_{1}=\mathrm{Ac}, \mathrm{R}_{2}=\mathrm{H}, \mathrm{R}_{3}=\alpha-\mathrm{CH}_{3}, \mathrm{R}_{4}=\alpha-\mathrm{OAc}$,

$\mathrm{R}_{5}=\mathrm{H}$ (euphornin $\left.\mathrm{B}\right)$

212. $R_{1}=A c, R_{2}=A c, R_{3}=\alpha-C_{3}, R_{4}=\alpha-$

OAc, $R_{5}=A c$ (euphornin D)

213.

E. helioscopia

Yamamura et al. (1989)

214. (euphornin E)

E. helioscopia

Yamamura et al. (1989)
215. $\mathrm{R}_{1}=\mathrm{OBu}, \mathrm{R}_{2}=\mathrm{OAc}$
216. $\mathrm{R}_{1}=\mathrm{OMeBu}, \mathrm{R}_{2}=\mathrm{OAc}$
217. $\mathrm{R}_{1}=\mathrm{OSal}, \mathrm{R}_{2}=\mathrm{OAc}$

E. helioscopia

Li et al. (2018a) 
Table 1 continued

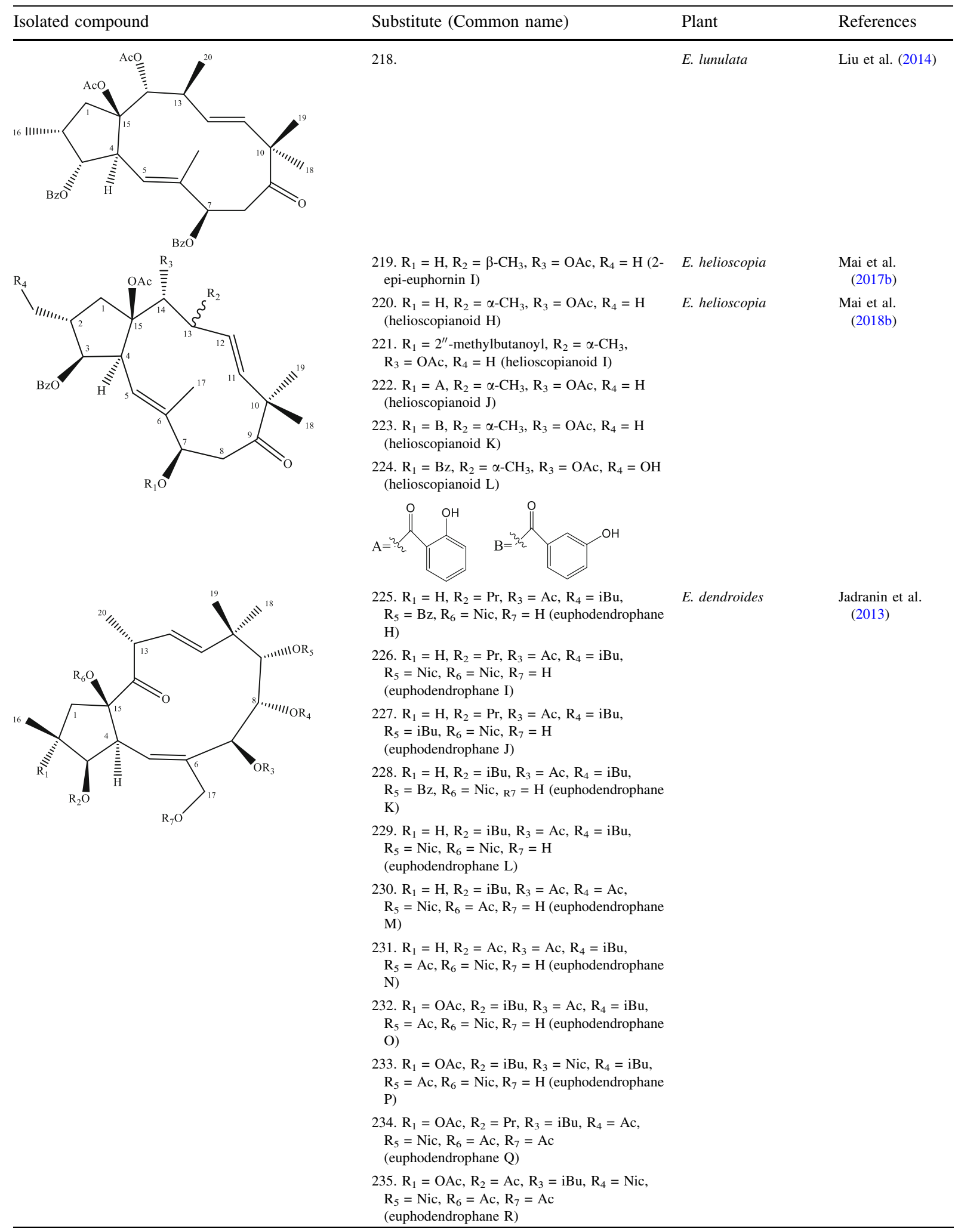


Table 1 continued

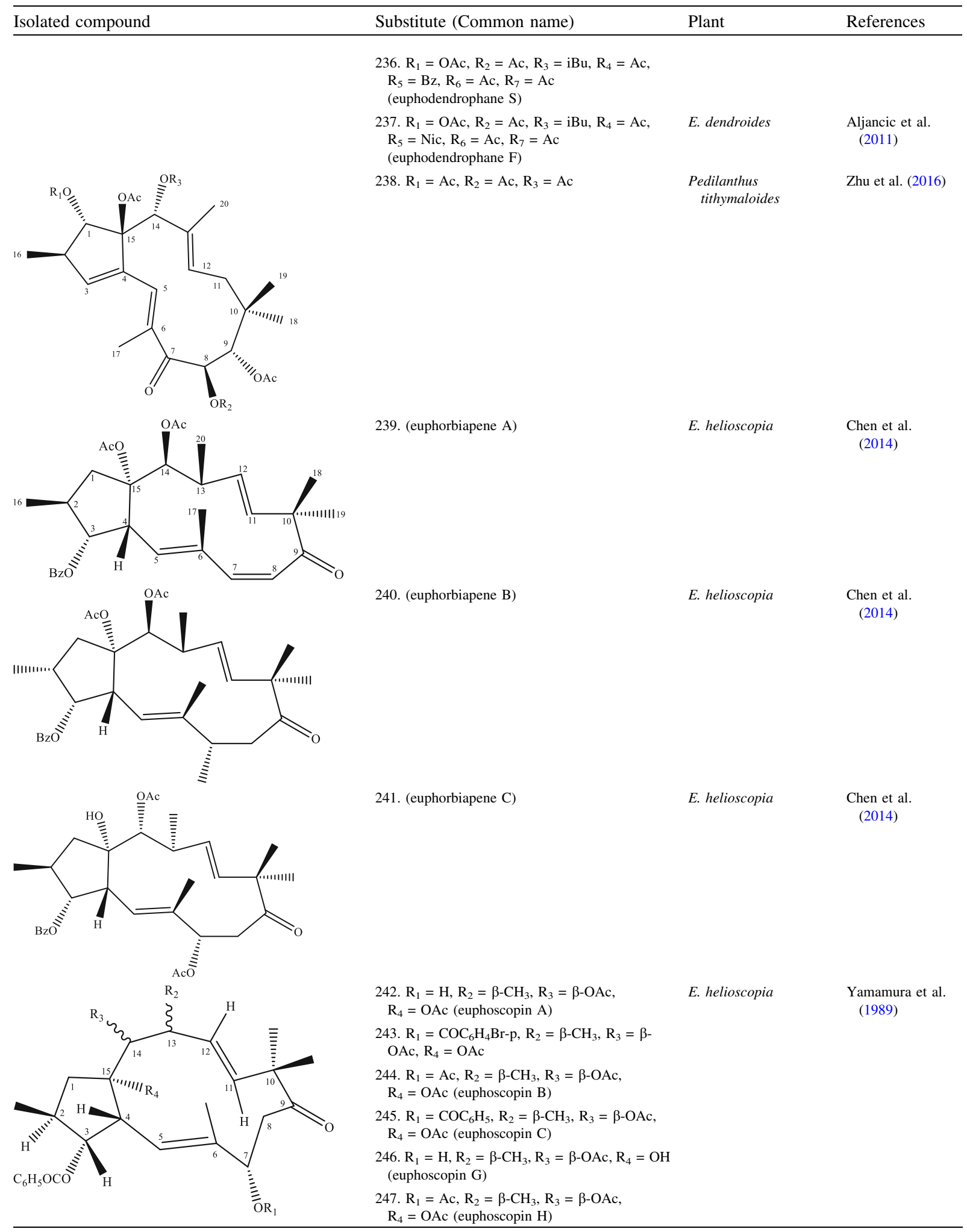


Table 1 continued

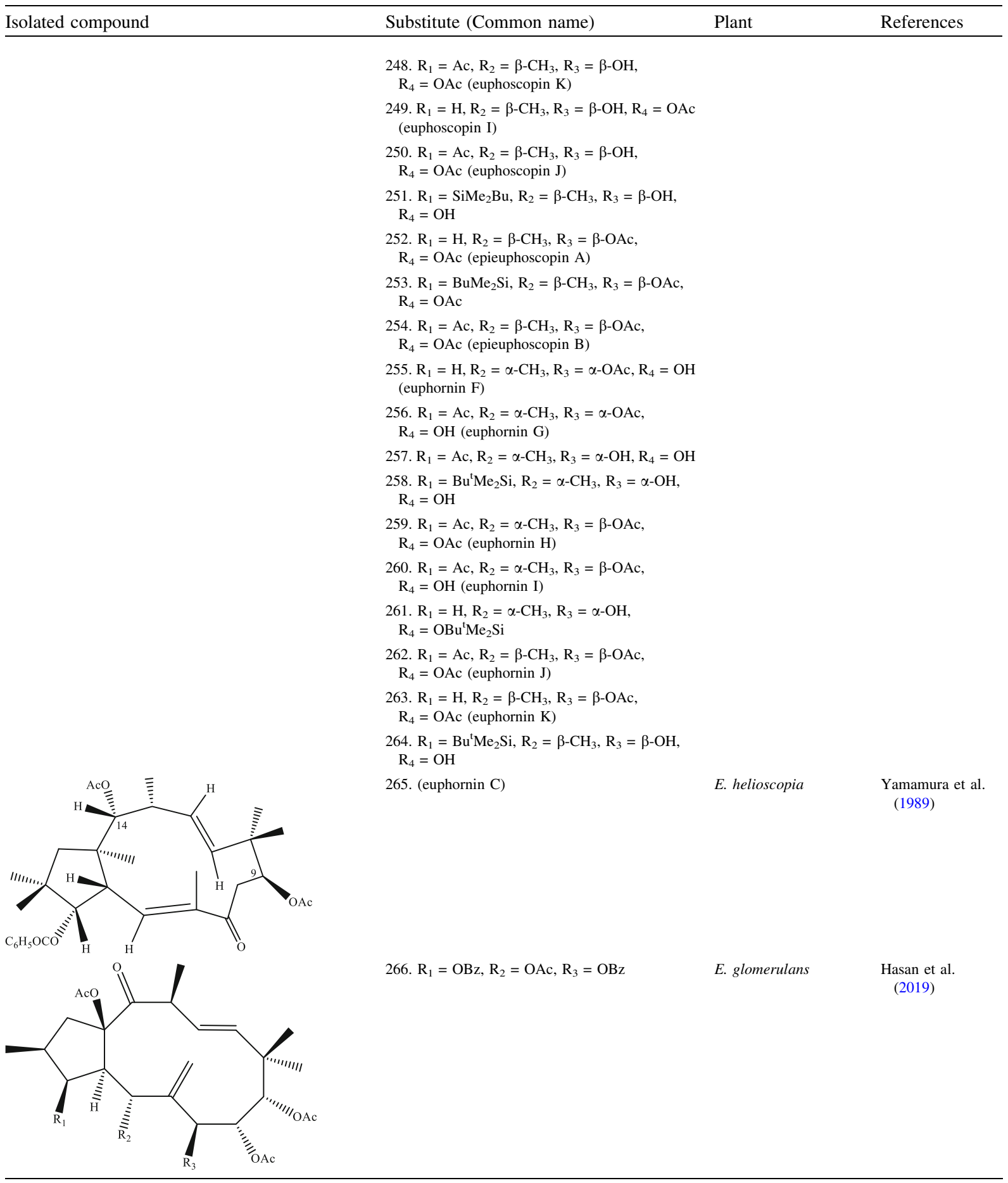


Table 1 continued

\begin{tabular}{|c|c|c|c|}
\hline \multirow[t]{10}{*}{ Isolated compound } & Substitute (Common name) & Plant & References \\
\hline & 267. (welwitschene) & E. welwitschii & $\begin{array}{l}\text { Reis et al. } \\
\text { (2015) }\end{array}$ \\
\hline & 268. (epoxywelwitschene) & E. welwitschii & $\begin{array}{l}\text { Reis et al. } \\
\text { (2015) }\end{array}$ \\
\hline & 269. & E. exigua & $\begin{array}{l}\text { Rédei et al. } \\
\text { (2015) }\end{array}$ \\
\hline & $\begin{array}{l}\text { 270. } R_{1}=R_{2}=R_{4}=A c, R_{3}=B z \text { (pubescene } A \text { ) } \\
\text { 271. } R_{1}=R_{2}=R_{4}=A c, R_{3}=B u \text { (pubescene } B \text { ) } \\
\text { 272. } R_{1}=H, R_{2}=R_{4}=A c, R_{3}=B z \text { (pubescene } \\
\text { C) }\end{array}$ & E. pubescens & $\begin{array}{c}\text { Valente et al. } \\
\text { (2003) }\end{array}$ \\
\hline & $\begin{array}{l}\text { 273. } \mathrm{R}_{1}=\mathrm{H}, \mathrm{R}_{2}=\mathrm{OAc}, \mathrm{R}_{3}=\mathrm{OPr}, \mathrm{R}_{4}=\alpha-\mathrm{Ac} \\
\mathrm{R}_{5}=\mathrm{OiBu}, \mathrm{R}_{6}=\alpha-\mathrm{OAc}, \mathrm{R}_{7}=\mathrm{Nic}, \mathrm{R}_{8}=\alpha- \\
\mathrm{CH}_{3}, \mathrm{R}_{9}=\mathrm{OH} \text { (nicaeenin } \mathrm{B} \text { ) }\end{array}$ & E. nicaeensis & $\begin{array}{l}\text { Krstić et al. } \\
\text { (2018) }\end{array}$ \\
\hline & $\begin{array}{l}\text { 274. } \mathrm{R}_{1}=\mathrm{H}, \mathrm{R}_{2}=\mathrm{ONic}, \mathrm{R}_{3}=\mathrm{OiBu}, \mathrm{R}_{4}=\alpha- \\
\mathrm{OAc}, \mathrm{R}_{5}=\mathrm{OiBu}, \mathrm{R}_{6}=\alpha-\mathrm{OAc}, \mathrm{R}_{7}=\mathrm{Ac}, \\
\mathrm{R}_{8}=\alpha-\mathrm{CH}_{3}, \mathrm{R}_{9}=\mathrm{OH} \text { (nicaeenin C) } \\
\text { 275. } \mathrm{R}_{1}=\mathrm{H}, \mathrm{R}_{2}=\mathrm{ONic}, \mathrm{R}_{3}=\mathrm{OPr}, \mathrm{R}_{4}=\alpha-\mathrm{OAc}, \\
\mathrm{R}_{5}=\mathrm{OiBu}, \mathrm{R}_{6}=\alpha-\mathrm{H}, \mathrm{R}_{7}=\mathrm{Nic}, \mathrm{R}_{8}=\alpha-\mathrm{CH}_{3}, \\
\mathrm{R}_{9}=\mathrm{OH}(\text { nicaeenin } \mathrm{D})\end{array}$ & & \\
\hline & $\begin{aligned} \text { 276. } \mathrm{R}_{1}=\mathrm{H}, \mathrm{R}_{2}=\mathrm{H}, \mathrm{R}_{3}=\mathrm{OPr}, \mathrm{R}_{4}=\alpha-\mathrm{OAc} \\
\mathrm{R}_{5}=\mathrm{OAc}, \mathrm{R}_{6}=\alpha-\mathrm{H}, \mathrm{R}_{7}=\mathrm{Nic}, \mathrm{R}_{8}=\alpha-\mathrm{CH}_{3} \\
\mathrm{R}_{9}=\mathrm{OAc}(\text { nicaeenin } \mathrm{E})\end{aligned}$ & E. nicaeensis & $\begin{array}{l}\text { Krstić et al. } \\
\text { (2018) }\end{array}$ \\
\hline & $\begin{array}{l}\text { 277. } \mathrm{R}_{1}=\mathrm{H}, \mathrm{R}_{2}=\mathrm{H}, \mathrm{R}_{3}=\mathrm{OPr}, \mathrm{R}_{4}=\alpha-\mathrm{OAc}, \\
\mathrm{R}_{5}=\mathrm{OAc}, \mathrm{R}_{6}=\alpha-\mathrm{H}, \mathrm{R}_{7}=\mathrm{Nic}, \mathrm{R}_{8}=\alpha-\mathrm{CH}_{3}, \\
\mathrm{R}_{9}=\mathrm{OAc}(\text { nicaeenin } \mathrm{F})\end{array}$ & & \\
\hline & $\begin{array}{l}\text { 278. } \mathrm{R}_{1}=\mathrm{H}, \mathrm{R}_{2}=\mathrm{H}, \mathrm{R}_{3}=\mathrm{OPr}, \mathrm{R}_{4}=\alpha-\mathrm{OAc} \\
\mathrm{R}_{5}=\mathrm{OiBu}, \mathrm{R}_{6}=\alpha-\mathrm{ONic}, \mathrm{R}_{7}=\mathrm{Ac}, \mathrm{R}_{8}=\alpha- \\
\mathrm{CH}_{3}, \mathrm{R}_{9}=\mathrm{OH} \text { (nicaeenin } \mathrm{G} \text { ) }\end{array}$ & & \\
\hline
\end{tabular}


Table 1 continued

\begin{tabular}{|c|c|c|c|}
\hline Isolated compound & Substitute (Common name) & Plant & References \\
\hline & $\begin{array}{l}\text { 279. } R_{1}=H, R_{2}=H, R_{3}=\text { OCin, } R_{4}=\alpha-O A c \\
R_{5}=H, R_{6}=\alpha-H, R_{7}=A c, R_{8}=\beta-C_{3} \\
R_{9}=O H\end{array}$ & E. taurinensis & $\begin{array}{l}\text { Rédei et al. } \\
\text { (2018) }\end{array}$ \\
\hline & $\begin{aligned} \text { 280. } \mathrm{R}_{1}=\mathrm{H}, \mathrm{R}_{2}=\mathrm{OiBu}, \mathrm{R}_{3}=\mathrm{OAc}, \mathrm{R}_{4}=\beta-\mathrm{OH} \\
\mathrm{R}_{5}=\mathrm{OAc}, \mathrm{R}_{6}=\beta-\mathrm{OAc}, \mathrm{R}_{7}=\mathrm{Bz}, \mathrm{R}_{8}=\beta-\mathrm{CH}_{3}, \\
\mathrm{R}_{9}=\mathrm{OH} \text { (guyonianin } \mathrm{G} \text { ) }\end{aligned}$ & E. guyoniana & $\begin{array}{l}\text { Kúsz et al. } \\
\text { (2016) }\end{array}$ \\
\hline & $\begin{array}{l}\text { 281. } \mathrm{R}_{1}=\mathrm{H}, \mathrm{R}_{2}=\mathrm{OiBu}, \mathrm{R}_{3}=\mathrm{OAc}, \mathrm{R}_{4}=\beta-\mathrm{OH} \\
\mathrm{R}_{5}=\mathrm{OiBu}, \mathrm{R}_{6}=\beta-\mathrm{OAc}, \mathrm{R}_{7}=\mathrm{Bz}, \mathrm{R}_{8}=\beta- \\
\mathrm{CH}_{3}, \mathrm{R}_{9}=\mathrm{OH} \text { (guyonianin } \mathrm{H} \text { ) }\end{array}$ & & \\
\hline & $\begin{array}{l}\text { 282. } \mathrm{R}_{1}=\mathrm{H}, \mathrm{R}_{2}=\mathrm{H}, \mathrm{R}_{3}=\mathrm{OAc}, \mathrm{R}_{4}=\alpha-\mathrm{OBz} \\
\mathrm{R}_{5}=\mathrm{OAc}, \mathrm{R}_{6}=\alpha \text {-OAc, } \mathrm{R}_{7}=\mathrm{Ac}, \mathrm{R}_{8}=\beta-\mathrm{CH}_{3} \\
\mathrm{R}_{9}=\mathrm{OH} \text { (euphorbesulin } \mathrm{D} \text { ) }\end{array}$ & E. esula & $\begin{array}{l}\text { Zhou et al. } \\
\text { (2016) }\end{array}$ \\
\hline & $\begin{array}{l}\text { 283. } \mathrm{R}_{1}=\mathrm{H}, \mathrm{R}_{2}=\mathrm{H}, \mathrm{R}_{3}=\mathrm{OBz}, \mathrm{R}_{4}=\alpha-\mathrm{OAc} \\
\mathrm{R}_{5}=\mathrm{OAc}, \mathrm{R}_{6}=\alpha \text {-OAc, } \mathrm{R}_{7}=\mathrm{Ac}, \mathrm{R}_{8}=\beta-\mathrm{CH}_{3}, \\
\mathrm{R}_{9}=\mathrm{OAc} \text { (euphorbesulin E) }\end{array}$ & & \\
\hline & $\begin{array}{l}\text { 284. } \mathrm{R}_{1}=\mathrm{H}, \mathrm{R}_{2}=\mathrm{H}, \mathrm{R}_{3}=\mathrm{OAc}, \mathrm{R}_{4}=\alpha-\mathrm{OAc} \\
\mathrm{R}_{5}=\mathrm{OAc}, \mathrm{R}_{6}=\alpha \text {-OAc, } \mathrm{R}_{7}=\mathrm{Ac}, \mathrm{R}_{8}=\beta-\mathrm{CH}_{3}, \\
\mathrm{R}_{9}=\mathrm{OAc} \text { (euphorbesulin F) }\end{array}$ & & \\
\hline & $\begin{aligned} \text { 285. } \mathrm{R}_{1}=\mathrm{H}, \mathrm{R}_{2}=\mathrm{H}, \mathrm{R}_{3}=\mathrm{OAc}, \mathrm{R}_{4}=\alpha-\mathrm{OBz} \\
\mathrm{R}_{5}=\mathrm{OBz}, \mathrm{R}_{6}=\alpha \text {-OAc, } \mathrm{R}_{7}=\mathrm{Ac}, \mathrm{R}_{8}=\beta-\mathrm{CH}_{3} \\
\mathrm{R}_{9}=\mathrm{OAc} \text { (euphorbesulin } \mathrm{G} \text { ) }\end{aligned}$ & & \\
\hline & $\begin{array}{l}\text { 286. } \mathrm{R}_{1}=\mathrm{H}, \mathrm{R}_{2}=\mathrm{H}, \mathrm{R}_{3}=\mathrm{OAc}, \mathrm{R}_{4}=\alpha-\mathrm{OAc} \\
\mathrm{R}_{5}=\mathrm{OBz}, \mathrm{R}_{6}=\alpha-\mathrm{OAc}, \mathrm{R}_{7}=\mathrm{Ac}, \mathrm{R}_{8}=\beta-\mathrm{CH}_{3}, \\
\left.\mathrm{R}_{9}=\mathrm{HOCH}_{2} \mathrm{CO}_{2} \text { (euphorbesulin } \mathrm{H}\right)\end{array}$ & & \\
\hline & $\begin{array}{l}\text { 287. } \mathrm{R}_{1}=\mathrm{H}, \mathrm{R}_{2}=\mathrm{H}, \mathrm{R}_{3}=\mathrm{OAc}, \mathrm{R}_{4}=\alpha-\mathrm{OBz} \\
\mathrm{R}_{5}=\mathrm{OAc}, \mathrm{R}_{6}=\alpha \text {-OAc, } \mathrm{R}_{7}=\mathrm{Ac}, \mathrm{R}_{8}=\beta-\mathrm{CH}_{3}, \\
\mathrm{R}_{9}=\mathrm{OAc} \text { (euphorbesulin I) }\end{array}$ & & \\
\hline & $\begin{array}{l}\text { 288. } \mathrm{R}_{1}=\mathrm{H}, \mathrm{R}_{2}=\mathrm{H}, \mathrm{R}_{3}=\mathrm{OAc}, \mathrm{R}_{4}=\alpha-\mathrm{OAc} \\
\mathrm{R}_{5}=\mathrm{OBz}, \mathrm{R}_{6}=\alpha-\mathrm{OH}, \mathrm{R}_{7}=\mathrm{Ac}, \mathrm{R}_{8}=\beta-\mathrm{CH}_{3} \\
\mathrm{R}_{9}=\mathrm{OAc} \text { (euphorbesulin } \mathrm{J} \text { ) }\end{array}$ & & \\
\hline & $\begin{array}{l}\text { 289. } \mathrm{R}_{1}=\mathrm{H}, \mathrm{R}_{2}=\mathrm{OAc}, \mathrm{R}_{3}=\mathrm{OAc}, \mathrm{R}_{4}=\alpha-\mathrm{OBz} \\
\mathrm{R}_{5}=\mathrm{OAc}, \mathrm{R}_{6}=\alpha-\mathrm{OAc}, \mathrm{R}_{7}=\mathrm{Ac}, \mathrm{R}_{8}=\beta-\mathrm{CH}_{3}, \\
\left.\mathrm{R}_{9}=\mathrm{OAc} \text { (euphorbesulin } \mathrm{K}\right)\end{array}$ & & \\
\hline & $\begin{aligned} 290 . \mathrm{R}_{1}=\mathrm{H}, \mathrm{R}_{2}=\mathrm{OAc}, \mathrm{R}_{3}=\mathrm{OBz}, \mathrm{R}_{4}=\alpha-\mathrm{OAc} \\
\mathrm{R}_{5}=\mathrm{OAc}, \mathrm{R}_{6}=\alpha-\mathrm{OAc}, \mathrm{R}_{7}=\mathrm{Ac}, \mathrm{R}_{8}=\beta-\mathrm{CH}_{3}, \\
\mathrm{R}_{9}=\mathrm{OAc} \text { (euphorbesulin } \mathrm{L} \text { ) }\end{aligned}$ & & \\
\hline & $\begin{array}{l}\text { 291. } \mathrm{R}_{1}=\mathrm{H}, \mathrm{R}_{2}=\mathrm{OH}, \mathrm{R}_{3}=\mathrm{OBz}, \mathrm{R}_{4}=\alpha-\mathrm{OAc} \\
\mathrm{R}_{5}=\mathrm{OBz}, \mathrm{R}_{6}=\alpha \text {-OAc, } \mathrm{R}_{7}=\mathrm{Ac}, \mathrm{R}_{8}=\beta-\mathrm{CH}_{3}, \\
\mathrm{R}_{9}=\mathrm{OAc} \text { (euphorbesulin } \mathrm{M} \text { ) }\end{array}$ & & \\
\hline & $\begin{aligned} \text { 292. } \mathrm{R}_{1}=\mathrm{OH}, \mathrm{R}_{2}=\mathrm{OH}, \mathrm{R}_{3}=\mathrm{OBz}, \mathrm{R}_{4}=\alpha-\mathrm{OBz} \\
\mathrm{R}_{5}=\mathrm{OBz}, \mathrm{R}_{6}=\alpha-\mathrm{OAc}, \mathrm{R}_{7}=\mathrm{Ac}, \mathrm{R}_{8}=\beta-\mathrm{CH}_{3}, \\
\mathrm{R}_{9}=\mathrm{OAc}(\text { euphorbesulin } \mathrm{N})\end{aligned}$ & & \\
\hline$\|$ & 293. (euphomelliferine) & E. mellifera & $\begin{array}{l}\text { Valente et al. } \\
\text { (2012) }\end{array}$ \\
\hline
\end{tabular}


Table 1 continued

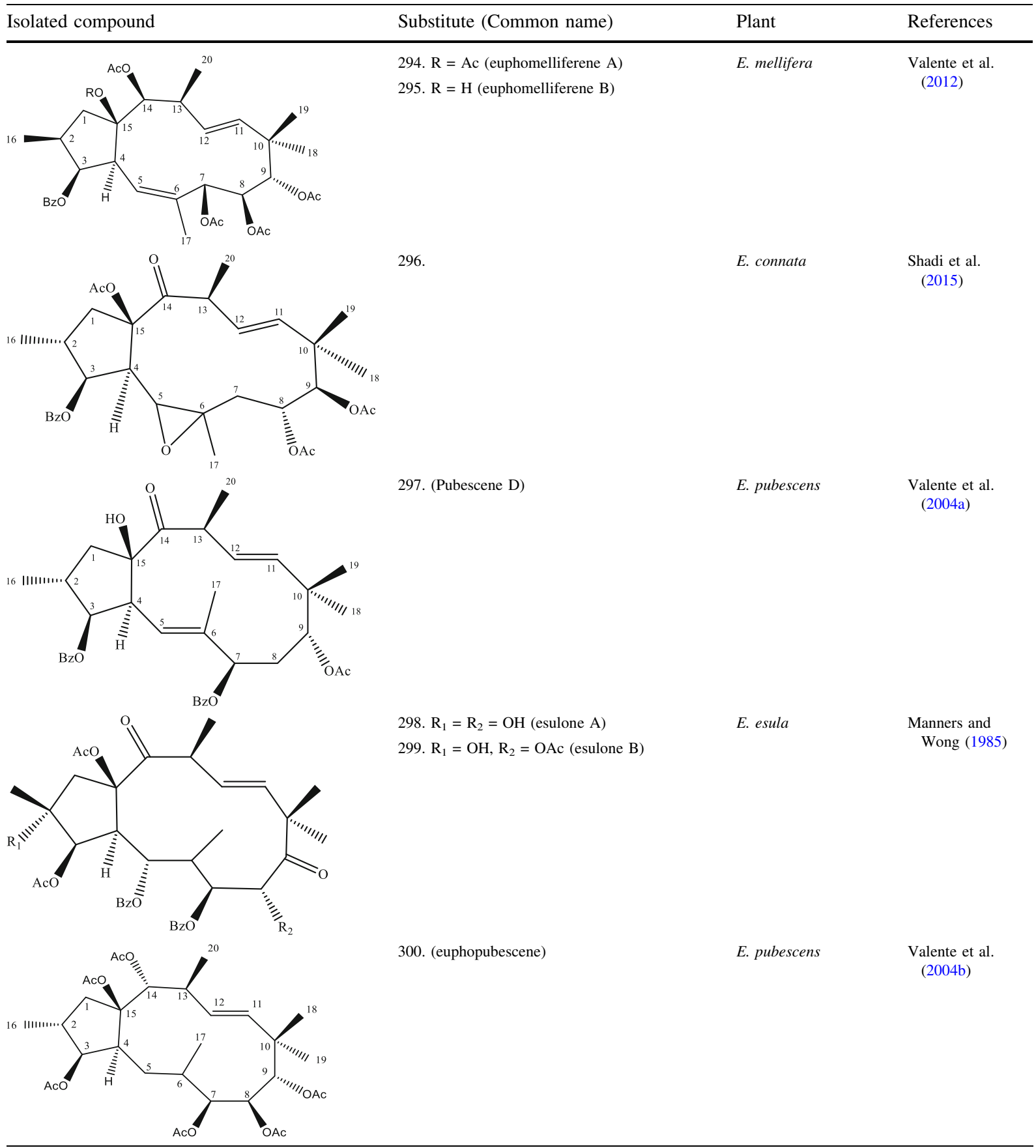


Table 1 continued

\begin{tabular}{|c|c|c|c|}
\hline Isolated compound & Substitute (Common name) & Plant & References \\
\hline & $\begin{array}{l}\text { 301. } \mathrm{R}_{1}=\mathrm{CH}_{3}, \mathrm{R}_{2}=\mathrm{OH}, \mathrm{R}_{3}=\mathrm{CH}_{3}, \mathrm{R}_{4}=\mathrm{OAc} \\
\mathrm{R}_{5}=\beta-\mathrm{CH}_{3}, \mathrm{R}_{6}=\mathrm{OAc}\end{array}$ & E. serrulata & $\begin{array}{l}\text { Hohmann et al. } \\
\text { (2002) }\end{array}$ \\
\hline & $\begin{array}{l}\text { 302. } \mathrm{R}_{1}=\mathrm{H}, \mathrm{R}_{2}=\mathrm{CH}_{3}, \mathrm{R}_{3}=\mathrm{CH}_{3}, \mathrm{R}_{4}=\mathrm{OH} \\
\mathrm{R}_{5}=\beta-\mathrm{CH}_{3}, \mathrm{R}_{6}=\mathrm{OAc}\end{array}$ & & \\
\hline & $\begin{array}{l}\text { 303. } \mathrm{R}_{1}=\mathrm{CH}_{3}, \mathrm{R}_{2}=\mathrm{OAc}, \mathrm{R}_{3}=\mathrm{OH}, \mathrm{R}_{4}=\mathrm{CH}_{3} \\
\mathrm{R}_{5}=\beta-\mathrm{CH}_{3}, \mathrm{R}_{6}=\mathrm{OH}\end{array}$ & E. serrulata & $\begin{array}{l}\text { Redei et al. } \\
\quad(2003)\end{array}$ \\
\hline & $\begin{array}{l}\text { 304. } \mathrm{R}_{1}=\mathrm{CH}_{3}, \mathrm{R}_{2}=\mathrm{OAc}, \mathrm{R}_{3}=\mathrm{OAc}, \mathrm{R}_{4}=\mathrm{CH}_{3} \\
\mathrm{R}_{5}=\beta-\mathrm{CH}_{3}, \mathrm{R}_{6}=\mathrm{OAc}\end{array}$ & & \\
\hline & $\begin{array}{l}\text { 305. } \mathrm{R}_{1}=\mathrm{H}, \mathrm{R}_{2}=\mathrm{CH}_{3}, \mathrm{R}_{3}=\mathrm{CH}_{3}, \mathrm{R}_{4}=\mathrm{OAc} \\
\mathrm{R}_{5}=\alpha-\mathrm{CH}_{3}, \mathrm{R}_{6}=\mathrm{OAc}\end{array}$ & & \\
\hline & $\begin{array}{l}\text { 306. } \mathrm{R}_{1}=\mathrm{CH}_{3}, \mathrm{R}_{2}=\mathrm{H}, \mathrm{R}_{3}=\mathrm{CH}_{3}, \mathrm{R}_{4}=\mathrm{OAc} \text {, } \\
\mathrm{R}_{5}=\alpha-\mathrm{CH}_{3}, \mathrm{R}_{6}=\mathrm{OAc} \text { (serrulatin B) }\end{array}$ & E. serrulata & $\begin{array}{l}\text { Hohmann et al. } \\
\text { (2000b) }\end{array}$ \\
\hline & $\begin{array}{l}\text { 307. } \mathrm{R}_{1}=\mathrm{H}, \mathrm{R}_{2}=\mathrm{CH}_{3}, \mathrm{R}_{3}=\mathrm{H}, \mathrm{R}_{4}=\mathrm{OBz} \text {, } \\
\mathrm{R}_{5}=\alpha-\mathrm{OAc}, \mathrm{R}_{6}=\mathrm{OAc}, \mathrm{R}_{7}=\mathrm{OAc}, \mathrm{R}_{8}=\mathrm{OAc}\end{array}$ & E. glomerulans & $\begin{array}{l}\text { Hasan et al. } \\
\quad(2019)\end{array}$ \\
\hline & $\begin{array}{l}\text { 308. } \mathrm{R}_{1}=\mathrm{H}, \mathrm{R}_{2}=\mathrm{CH}_{3}, \mathrm{R}_{3}=\mathrm{H}, \mathrm{R}_{4}=\mathrm{OAc} \\
\mathrm{R}_{5}=\alpha-\mathrm{OBz}, \mathrm{R}_{6}=\mathrm{OAc}, \mathrm{R}_{7}=\mathrm{OH}, \mathrm{R}_{8}=\mathrm{OAc}\end{array}$ & & \\
\hline & $\begin{array}{l}\text { 309. } \mathrm{R}_{1}=\mathrm{H}, \mathrm{R}_{2}=\mathrm{CH}_{3}, \mathrm{R}_{3}=\mathrm{H}, \mathrm{R}_{4}=\mathrm{OBz} \text {, } \\
\mathrm{R}_{5}=\alpha \text {-OAc, } \mathrm{R}_{6}=\mathrm{OAc}, \mathrm{R}_{7}=\mathrm{OH}, \mathrm{R}_{8}=\mathrm{OAc}\end{array}$ & & \\
\hline & $\begin{array}{l}\text { 310. } \mathrm{R}_{1}=\mathrm{H}, \mathrm{R}_{2}=\mathrm{CH}_{3}, \mathrm{R}_{3}=\mathrm{H}, \mathrm{R}_{4}=\mathrm{OAc} \\
\mathrm{R}_{5}=\alpha-\mathrm{OBz}, \mathrm{R}_{6}=\mathrm{OAc}, \mathrm{R}_{7}=\mathrm{H}, \mathrm{R}_{8}=\mathrm{OAc}\end{array}$ & & \\
\hline $\mathrm{R}_{5}$ & $\begin{array}{l}\text { 311. } \mathrm{R}_{1}=\mathrm{H}, \mathrm{R}_{2}=\mathrm{CH}_{3}, \mathrm{R}_{3}=\mathrm{H}, \mathrm{R}_{4}=\mathrm{OBz} \\
\mathrm{R}_{5}=\alpha-\mathrm{OAc}, \mathrm{R}_{6}=\mathrm{OAc}, \mathrm{R}_{7}=\mathrm{H}, \mathrm{R}_{8}=\mathrm{OAc}\end{array}$ & & \\
\hline & $\begin{array}{l}\text { 312. } \mathrm{R}_{1}=\mathrm{H}, \mathrm{R}_{2}=\mathrm{CH}_{3}, \mathrm{R}_{3}=\mathrm{OH}, \mathrm{R}_{4}=\mathrm{OBz} \\
\mathrm{R}_{5}=\alpha-\mathrm{OAc}, \mathrm{R}_{6}=\mathrm{OBz}, \mathrm{R}_{7}=\mathrm{OAc}, \mathrm{R}_{8}=\mathrm{OH}\end{array}$ & & \\
\hline & $\begin{array}{l}\text { 313. } \mathrm{R}_{1}=\mathrm{H}, \mathrm{R}_{2}=\mathrm{CH}_{3}, \mathrm{R}_{3}=\mathrm{H}, \mathrm{R}_{4}=\mathrm{OBz} \\
\mathrm{R}_{5}=\alpha-\mathrm{OAc}, \mathrm{R}_{6}=\mathrm{OBz}, \mathrm{R}_{7}=\mathrm{OH}, \mathrm{R}_{8}=\mathrm{OAc}\end{array}$ & & \\
\hline & $\begin{array}{l}\text { 314. } \mathrm{R}_{1}=\mathrm{H}, \mathrm{R}_{2}=\mathrm{CH}_{3}, \mathrm{R}_{3}=\mathrm{OH}, \mathrm{R}_{4}=\mathrm{OBz} \text {, } \\
\mathrm{R}_{5}=\alpha-\mathrm{OAc}, \mathrm{R}_{6}=\mathrm{OAc}, \mathrm{R}_{7}=\mathrm{OiBu}, \mathrm{R}_{8}=\mathrm{OH}\end{array}$ & & \\
\hline & $\begin{array}{l}\text { 315. } \mathrm{R}_{1}=\mathrm{H}, \mathrm{R}_{2}=\mathrm{CH}_{3}, \mathrm{R}_{3}=\mathrm{OAc}, \mathrm{R}_{4}=\mathrm{OBz} \\
\mathrm{R}_{5}=\alpha-\mathrm{OAc}, \mathrm{R}_{6}=\mathrm{OAc}, \mathrm{R}_{7}=\mathrm{OiBu}, \mathrm{R}_{8}=\mathrm{OH}\end{array}$ & & \\
\hline & $\begin{array}{l}\text { 316. } \mathrm{R}_{1}=\mathrm{H}, \mathrm{R}_{2}=\mathrm{CH}_{3}, \mathrm{R}_{3}=\mathrm{OAc}, \mathrm{R}_{4}=\mathrm{OBz} \\
\mathrm{R}_{5}=\alpha-\mathrm{OAc}, \mathrm{R}_{6}=\mathrm{OiBu}, \mathrm{R}_{7}=\mathrm{OAc}, \mathrm{R}_{8}=\mathrm{OH}\end{array}$ & & \\
\hline & $\begin{array}{l}\text { 317. } \mathrm{R}_{1}=\mathrm{H}, \mathrm{R}_{2}=\mathrm{CH}_{3}, \mathrm{R}_{3}=\mathrm{H}, \mathrm{R}_{4}=\mathrm{OBz} \\
\mathrm{R}_{5}=\alpha-\mathrm{OAc}, \mathrm{R}_{6}=\mathrm{OAc}, \mathrm{R}_{7}=\mathrm{OH}, \mathrm{R}_{8}=\mathrm{OH}\end{array}$ & & \\
\hline & $\begin{array}{l}\text { 318. } \mathrm{R}_{1}=\mathrm{H}, \mathrm{R}_{2}=\mathrm{CH}_{3}, \mathrm{R}_{3}=\mathrm{H}, \mathrm{R}_{4}=\mathrm{OAc} \text {, } \\
\mathrm{R}_{5}=\alpha-\mathrm{OBz}, \mathrm{R}_{6}=\mathrm{OAc}, \mathrm{R}_{7}=\mathrm{OBz}, \mathrm{R}_{8}=\mathrm{OAc}\end{array}$ & & \\
\hline & $\begin{array}{l}\text { 319. } \mathrm{R}_{1}=\mathrm{H}, \mathrm{R}_{2}=\mathrm{CH}_{3}, \mathrm{R}_{3}=\mathrm{H}, \mathrm{R}_{4}=\mathrm{OBz} \\
\mathrm{R}_{5}=\alpha-\mathrm{OAc}, \mathrm{R}_{6}=\mathrm{OBz}, \mathrm{R}_{7}=\mathrm{OAc}, \mathrm{R}_{8}=\mathrm{OAc}\end{array}$ & & \\
\hline & $\begin{aligned} \text { 320. } \mathrm{R}_{1}=\mathrm{H}, \mathrm{R}_{2}=\mathrm{CH}_{3}, \mathrm{R}_{3}=\mathrm{H} \\
\mathrm{R}_{4}=\mathrm{CH}_{3} \mathrm{COCH}_{2} \mathrm{CO}_{2}, \mathrm{R}_{5}=\alpha-\mathrm{OAc}, \mathrm{R}_{6}=\mathrm{OBz}, \\
\mathrm{R}_{7}=\mathrm{OAc}, \mathrm{R}_{8}=\mathrm{OH}\end{aligned}$ & & \\
\hline & $\begin{array}{l}\text { 321. } \mathrm{R}_{1}=\mathrm{H}, \mathrm{R}_{2}=\mathrm{H}, \mathrm{R}_{3}=\mathrm{CH}_{3}, \mathrm{R}_{4}=\mathrm{OBz} \\
\mathrm{R}_{5}=\alpha-\mathrm{OAc}, \mathrm{R}_{6}=\mathrm{H}, \mathrm{R}_{7}=\mathrm{H}, \mathrm{R}_{8}=\mathrm{OH}\end{array}$ & E. helioscopia & Li et al. (2018a) \\
\hline & $\begin{array}{l}\text { 322. } \mathrm{R}_{1}=\mathrm{H}, \mathrm{R}_{2}=\mathrm{CH}_{3}, \mathrm{R}_{3}=\mathrm{H}, \mathrm{R}_{4}=\mathrm{OH} \\
\mathrm{R}_{5}=\beta-\mathrm{OBz}, \mathrm{R}_{6}=\mathrm{H}, \mathrm{R}_{7}=\mathrm{H}, \mathrm{R}_{8}=\mathrm{OAc}\end{array}$ & E. sororia & Hu et al. (2018) \\
\hline & $\begin{array}{l}\text { 323. } \mathrm{R}_{1}=\mathrm{OH}, \mathrm{R}_{2}=\mathrm{CH}_{3}, \mathrm{R}_{3}=\mathrm{OH}, \mathrm{R}_{4}=\mathrm{OAc} \\
\mathrm{R}_{5}=\alpha-\mathrm{OBz}, \mathrm{R}_{6}=\mathrm{OBz}, \mathrm{R}_{7}=\mathrm{OH}, \mathrm{R}_{8}=\mathrm{OAc} \\
\text { (kanesulone A) }\end{array}$ & E. kansui & Lee et al. (2016) \\
\hline & $\begin{array}{l}\text { 324. } \mathrm{R}_{1}=\mathrm{OAc}, \mathrm{R}_{2}=\mathrm{CH}_{3}, \mathrm{R}_{3}=\mathrm{OH}, \mathrm{R}_{4}=\mathrm{OAc} \\
\mathrm{R}_{5}=\alpha-\mathrm{OBz}, \mathrm{R}_{6}=\mathrm{OBz}, \mathrm{R}_{7}=\mathrm{OAc}, \mathrm{R}_{8}=\mathrm{OH} \\
\text { (kanesulone } \mathrm{B} \text { ) }\end{array}$ & & \\
\hline & 325. $\mathrm{R}_{1}=$ Tig & E. characias & Seip and Hecker \\
\hline & 326. $\mathrm{R}_{1}=\mathrm{Bz}$ & & (1984) \\
\hline
\end{tabular}


Table 1 continued

Isolated compound
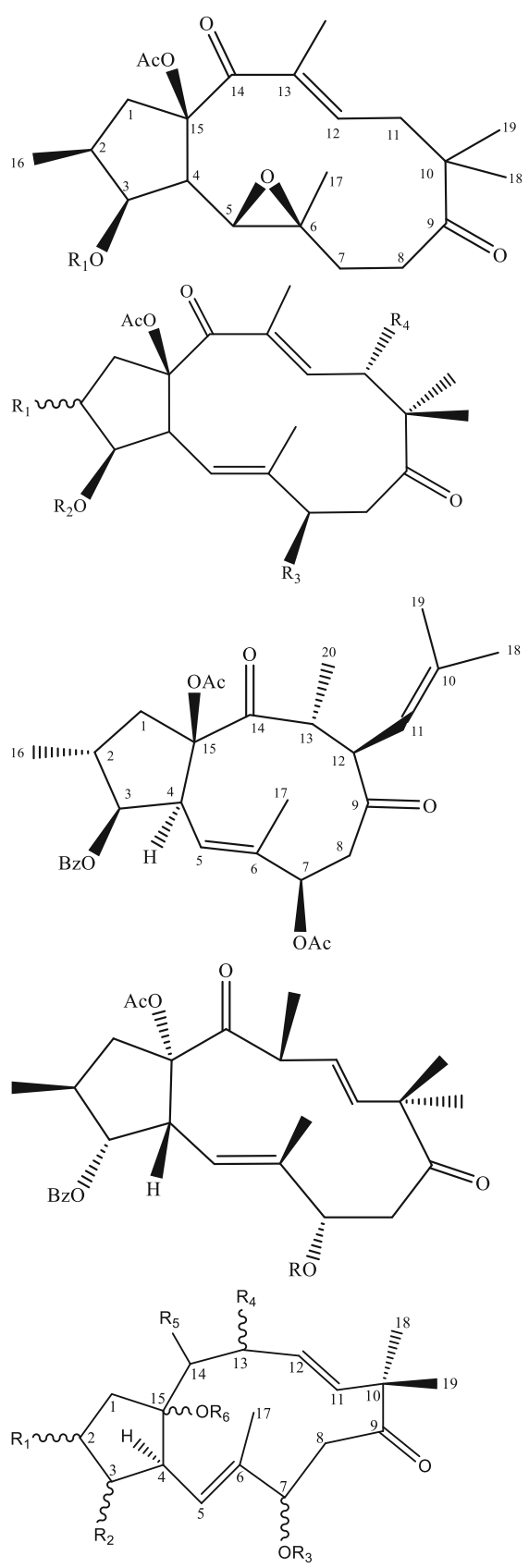

Substitute (Common name)

Plant

References

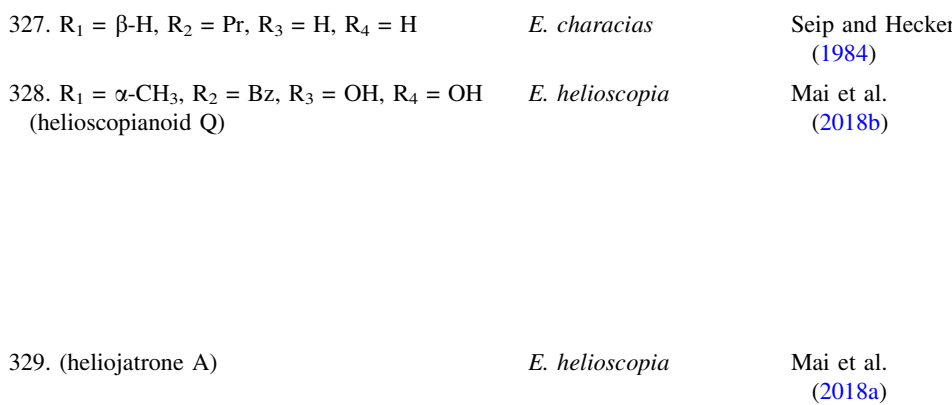

330. $\mathrm{R}=\mathrm{Bz}$ (euphorbiapene D)

E. helioscopia

Chen et al.

(2014)

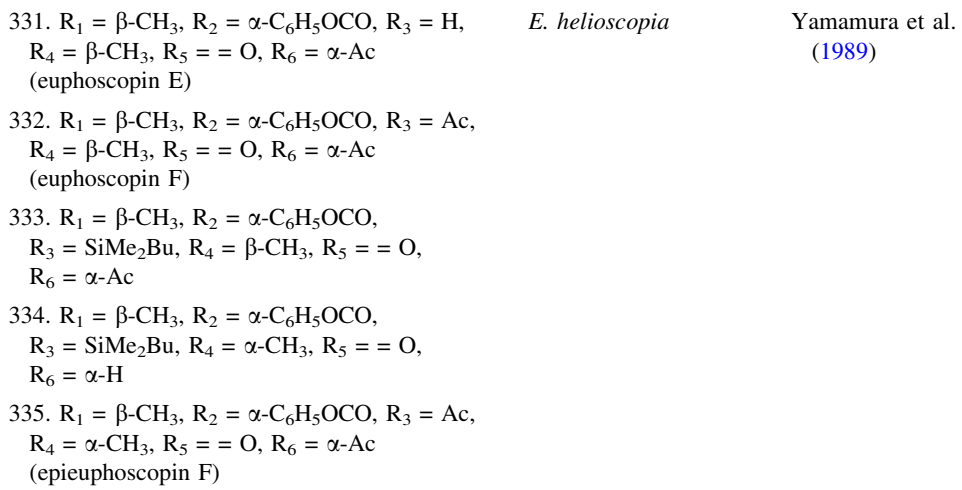


Table 1 continued

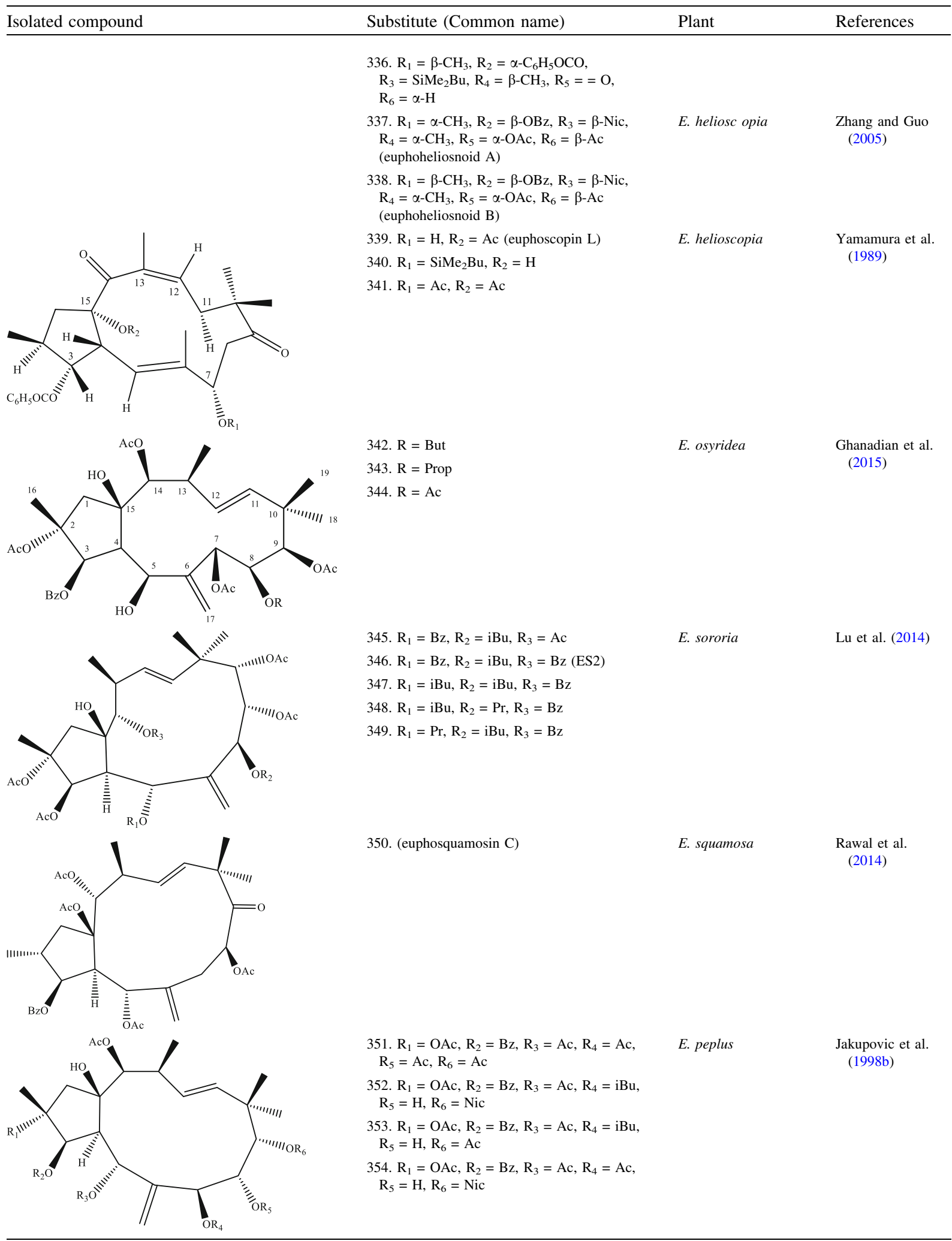


Table 1 continued
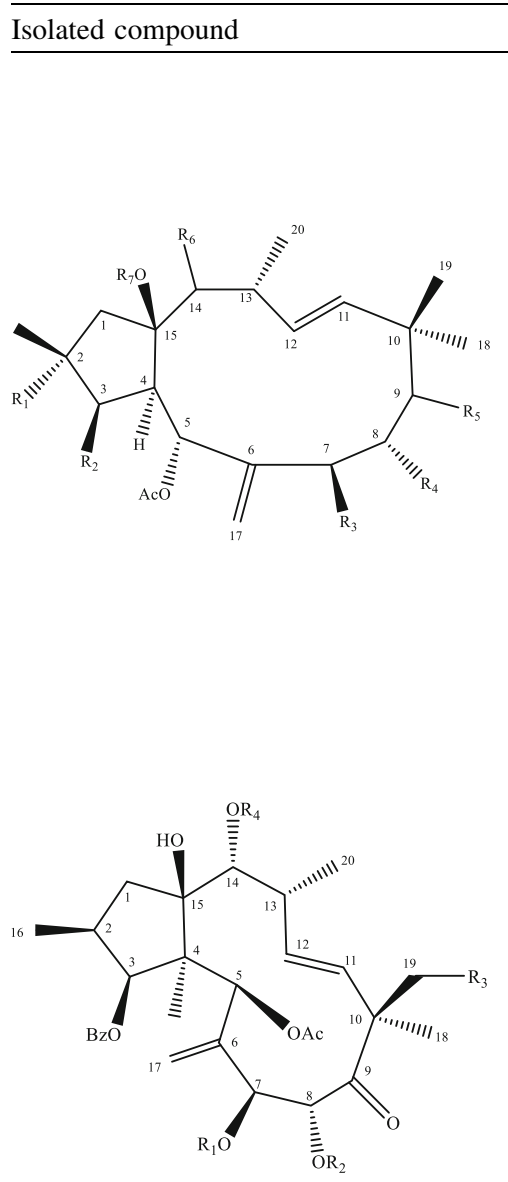

Substitute (Common name)

Plant

References

355. $\mathrm{R}_{1}=\mathrm{OAc}, \mathrm{R}_{2}=\mathrm{Bz}, \mathrm{R}_{3}=\mathrm{Ac}, \mathrm{R}_{4}=\mathrm{Ac}$,

$\mathrm{R}_{5}=\mathrm{H}, \mathrm{R}_{6}=\mathrm{Ac}$

356. $R_{1}=H, R_{2}=H, R_{3}=M e b u, R_{4}=i B u$,

$\mathrm{R}_{5}=$ Mebu, $\mathrm{R}_{6}=\mathrm{H}$

357. $\mathrm{R}_{1}=\mathrm{H}, \mathrm{R}_{2}=\mathrm{OBz}, \mathrm{R}_{3}=\mathrm{OAc}, \mathrm{R}_{4}=\mathrm{OAc}$,

$\mathrm{R}_{5}==\mathrm{O}, \mathrm{R}_{6}=\alpha-\mathrm{OAc}, \mathrm{R}_{7}=\mathrm{H}$

E. segetalis

Jakupovic et al. (1998a)

358. $\mathrm{R}_{1}=\mathrm{H}, \mathrm{R}_{2}=\mathrm{OBz}, \mathrm{R}_{3}=\mathrm{OAc}, \mathrm{R}_{4}=\mathrm{OiBu}$,

$\mathrm{R}_{5}==\mathrm{O}, \mathrm{R}_{6}=\alpha-\mathrm{OAc}, \mathrm{R}_{7}=\mathrm{H}$

359. $\mathrm{R}_{1}=\mathrm{H}, \mathrm{R}_{2}=\mathrm{OBz}, \mathrm{R}_{3}=\mathrm{OAc}, \mathrm{R}_{4}=\mathrm{OH}$,

$\mathrm{R}_{5}==\mathrm{O}, \mathrm{R}_{6}=\alpha-\mathrm{OH}, \mathrm{R}_{7}=\mathrm{H}$

360. $\mathrm{R}_{1}=\mathrm{H}, \mathrm{R}_{2}=\mathrm{OBz}, \mathrm{R}_{3}=\mathrm{OAc}, \mathrm{R}_{4}=\mathrm{OAc}$,

$\mathrm{R}_{5}==\mathrm{O}, \mathrm{R}_{6}=\alpha-\mathrm{OH}, \mathrm{R}_{7}=\mathrm{H}$

361. $\mathrm{R}_{1}=\mathrm{H}, \mathrm{R}_{2}=\mathrm{OBz}, \mathrm{R}_{3}=\mathrm{OAc}, \mathrm{R}_{4}=\mathrm{OH}$,

$\mathrm{R}_{5}==\mathrm{O}, \mathrm{R}_{6}=\alpha-\mathrm{OAc}, \mathrm{R}_{7}=\mathrm{H}$

362. $\mathrm{R}_{1}=\mathrm{H}, \mathrm{R}_{2}=\mathrm{OBz}, \mathrm{R}_{3}=\mathrm{OAc}, \mathrm{R}_{4}=\mathrm{H}$,

$\mathrm{R}_{5}==\mathrm{O}, \mathrm{R}_{6}=\alpha$-OAc, $\mathrm{R}_{7}=\mathrm{H}$

363. $\mathrm{R}_{1}=\mathrm{OAc}, \mathrm{R}_{2}=\mathrm{PhO}_{2} \mathrm{CH}_{2} \mathrm{CO}_{2} \mathrm{C}$,

$\mathrm{R}_{3}=\mathrm{OiBu}, \mathrm{R}_{4}=\mathrm{OAc}, \mathrm{R}_{5}=\alpha-\mathrm{OAc}, \mathrm{R}_{6}==\mathrm{O}$,

$$
\mathrm{R}_{7}=\mathrm{H}
$$

E. segetalis

Appendino et al. (1998)

364. $\mathrm{R}_{1}=\mathrm{H}, \mathrm{R}_{2}=\mathrm{OBz}, \mathrm{R}_{3}=\mathrm{H}, \mathrm{R}_{4}=\mathrm{H}$,

$\mathrm{R}_{5}=\mathrm{O}, \mathrm{R}_{6}=\alpha-\mathrm{OAc}, \mathrm{R}_{7}=\mathrm{H}($ guyonianin $\mathrm{E})$

365. $\mathrm{R}_{1}=\mathrm{H}, \mathrm{R}_{2}=\mathrm{Ac}, \mathrm{R}_{3}=\mathrm{H}, \mathrm{R}_{4}=\mathrm{H}$

E. guyoniana

.

E. semiperfoliata

(1)

366. $R_{1}=A c, R_{2}=H, R_{3}=O H, R_{4}=A c$

367. $R_{1}=A c, R_{2}=i B u, R_{3}=H, R_{4}=H$

368. $\mathrm{R}_{1}=\mathrm{Ac}, \mathrm{R}_{2}=\mathrm{MeBu}, \mathrm{R}_{3}=\mathrm{H}, \mathrm{R}_{4}=\mathrm{H}$

E. amygdaloides

E. amygdaloides

Jakupovic et al. (1998a)

Hegazy et al. (2010)

Nothias-Scaglia et al. (2015b)

Nothias-Scaglia et al. (2014)

369. $\mathrm{R}_{1}=\mathrm{H}, \mathrm{R}_{2}=\mathrm{Ac}, \mathrm{R}_{3}=\mathrm{OBz}, \mathrm{R}_{4}=\mathrm{OBz}$

E. kansui

Pan et al. (2004)

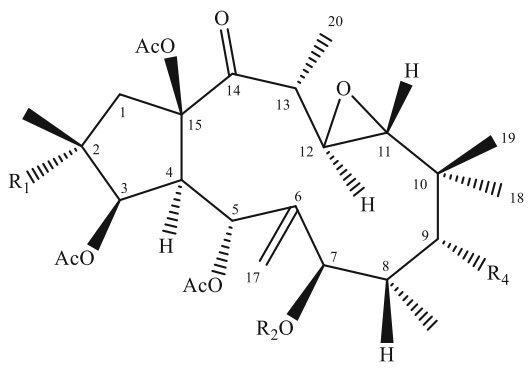
(kansuinin F)

370. $\mathrm{R}_{1}=\mathrm{H}, \mathrm{R}_{2}=\mathrm{Ac}, \mathrm{R}_{3}=\mathrm{H}, \mathrm{R}_{4}=\mathrm{ONic}$

(kansuinin $\mathrm{G}$ )

371. $\mathrm{R}_{1}=\mathrm{H}, \mathrm{R}_{2}=\mathrm{Ac}, \mathrm{R}_{3}=\mathrm{OBz}, \mathrm{R}_{4}=\mathrm{ONic}$ (kansuinin E)

372. $\mathrm{R}_{1}=\mathrm{OAc}, \mathrm{R}_{2}=\mathrm{iBu}, \mathrm{R}_{3}=\mathrm{OiBu}, \mathrm{R}_{4}=\mathrm{OAc}$

373. $\mathrm{R}_{1}=\mathrm{OAc}, \mathrm{R}_{2}=\mathrm{Ac}, \mathrm{R}_{3}=\mathrm{OiBu}, \mathrm{R}_{4}=\mathrm{OAc}$

374. $R_{1}=O A c, R_{2}=i B u, R_{3}=O A c, R_{4}=O A c$ (esulatin A)

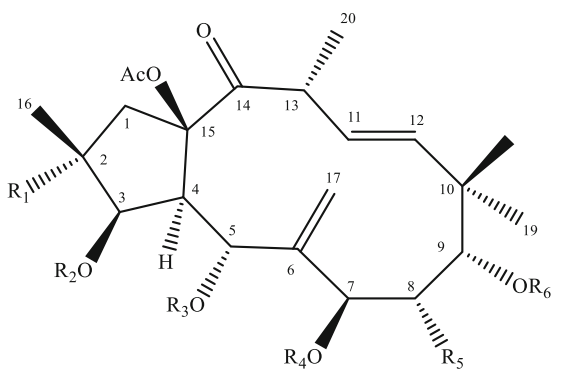

375. $\mathrm{R}_{1}=\mathrm{H}, \mathrm{R}_{2}=\mathrm{Bz}, \mathrm{R}_{3}=\mathrm{Ac}, \mathrm{R}_{4}=\mathrm{Ac}$,

$\mathrm{R}_{5}=\mathrm{OAc}, \mathrm{R}_{6}=\mathrm{Ac}$

E. salicifolia

Hohmann et al. (2001a)

E. esula

Hohmann et al. (1997)

376. $R_{1}=H, R_{2}=A c, R_{3}=B z, R_{4}=A c$,

$\mathrm{R}_{5}=\mathrm{OAc}, \mathrm{R}_{6}=\mathrm{Ac}$

377. $\mathrm{R}_{1}=\mathrm{OAc}, \mathrm{R}_{2}=\mathrm{Ac}, \mathrm{R}_{3}=A c, \mathrm{R}_{4}=\mathrm{Bz}$,

$\mathrm{R}_{5}=\mathrm{OAc}, \mathrm{R}_{6}=\mathrm{Ac}$

Liu and Tan

(2001)

378. $\mathrm{R}_{1}=\mathrm{H}, \mathrm{R}_{2}=A c, \mathrm{R}_{3}=A c, \mathrm{R}_{4}=\mathrm{Bz}$,

$\mathrm{R}_{5}=\mathrm{OAc}, \mathrm{R}_{6}=\mathrm{Ac}$

379. $R_{1}=O B z, R_{2}=A c, R_{3}=A c, R_{4}=A c$,

$\mathrm{R}_{5}=\mathrm{OAc}, \mathrm{R}_{6}=\mathrm{Ac}$

380. $R_{1}=$ OAc, $R_{2}=B z, R_{3}=A c, R_{4}=i B u$,

$\mathrm{R}_{5}=\mathrm{OAc}, \mathrm{R}_{6}=\mathrm{Nic}$

E. mongolica

Hohmann et al.

(2003b)

381. $\mathrm{R}_{1}=\mathrm{H}, \mathrm{R}_{2}=\mathrm{Ac}, \mathrm{R}_{3}=\mathrm{Ac}, \mathrm{R}_{4}=\mathrm{Ac}$,

$\mathrm{R}_{5}=\mathrm{H}, \mathrm{R}_{6}=\mathrm{Nic}$

E. peplus

Hohmann et al. (2000a)

E. peplus

Hohmann et al. (1999b) 
Table 1 continued

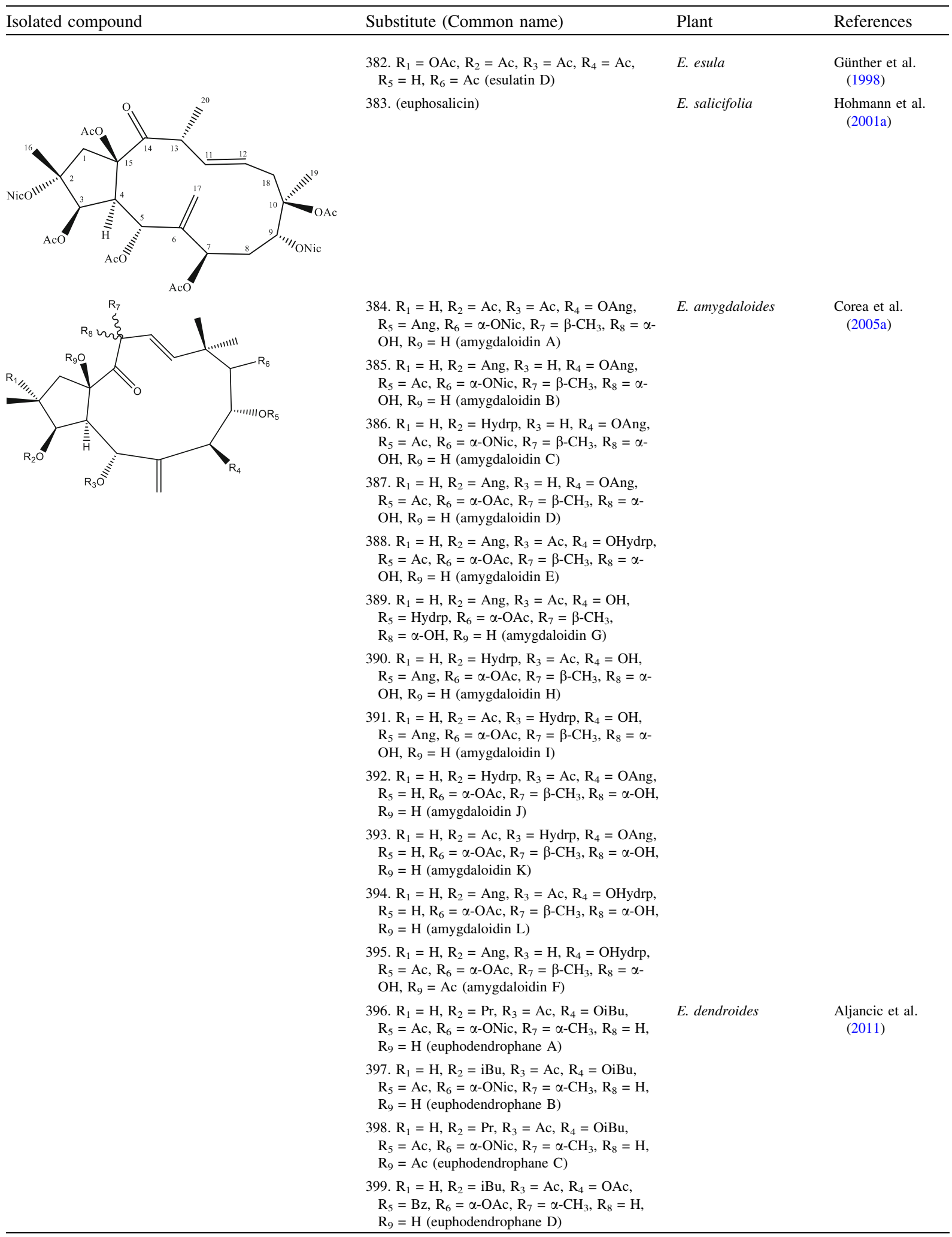


Table 1 continued

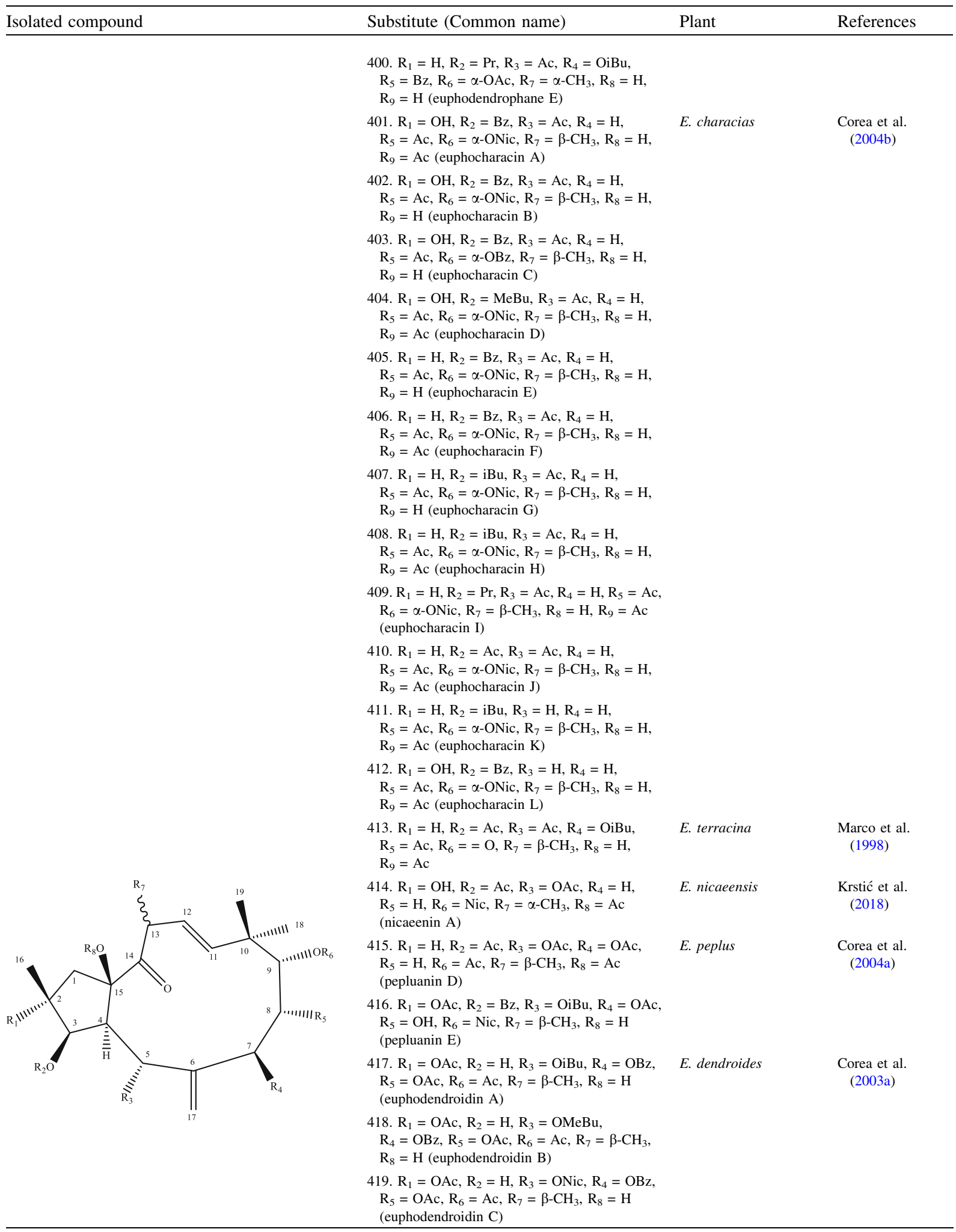


Table 1 continued

Isolated compound

Substitute (Common name)

Plant

References

420. $\mathrm{R}_{1}=\mathrm{H}, \mathrm{R}_{2}=\mathrm{H}, \mathrm{R}_{3}=\mathrm{OiBu}, \mathrm{R}_{4}=\mathrm{OBz}$,

$\mathrm{R}_{5}=\mathrm{OAc}, \mathrm{R}_{6}=\mathrm{Ac}, \mathrm{R}_{7}=\beta-\mathrm{CH}_{3}, \mathrm{R}_{8}=\mathrm{H}$

(euphodendroidin $\mathrm{D}$ )

421. $\mathrm{R}_{1}=\mathrm{H}, \mathrm{R}_{2}=\mathrm{Ac}, \mathrm{R}_{3}=\mathrm{OiBu}, \mathrm{R}_{4}=\mathrm{OBz}$,

$\mathrm{R}_{5}=\mathrm{OAc}, \mathrm{R}_{6}=\mathrm{Ac}, \mathrm{R}_{7}=\beta-\mathrm{CH}_{3}, \mathrm{R}_{8}=\mathrm{H}$

(euphodendroidin $\mathrm{E}$ )

422. $\mathrm{R}_{1}=\mathrm{OH}, \mathrm{R}_{2}=\mathrm{Ac}, \mathrm{R}_{3}=\mathrm{OiBu}, \mathrm{R}_{4}=\mathrm{OBz}$,

$\mathrm{R}_{5}=\mathrm{OAc}, \mathrm{R}_{6}=\mathrm{Ac}, \mathrm{R}_{7}=\beta-\mathrm{CH}_{3}, \mathrm{R}_{8}=\mathrm{H}$

(euphodendroidin $\mathrm{F}$ )

423. $R_{1}=O A c, R_{2}=$ Nic, $R_{3}=O A c, R_{4}=O i B u$,

$\mathrm{R}_{5}=\mathrm{OAc}, \mathrm{R}_{6}=\mathrm{Ac}, \mathrm{R}_{7}=\beta-\mathrm{CH}_{3}, \mathrm{R}_{8}=\mathrm{H}$

(euphodendroidin $\mathrm{G}$ )

424. $\mathrm{R}_{1}=\mathrm{H}, \mathrm{R}_{2}=\mathrm{Bz}, \mathrm{R}_{3}=\mathrm{OAc}, \mathrm{R}_{4}=\mathrm{OiBu}$,

$\mathrm{R}_{5}=\mathrm{OAc}, \mathrm{R}_{6}=\mathrm{Ac}, \mathrm{R}_{7}=\beta-\mathrm{CH}_{3}, \mathrm{R}_{8}=\mathrm{H}$

(euphodendroidin $\mathrm{H}$ )

425. $\mathrm{R}_{1}=\mathrm{OMB}\left(2-\right.$ MethylButyrate), $\mathrm{R}_{2}=\mathrm{H}$, $\mathrm{R}_{3}=\mathrm{OMB}, \mathrm{R}_{4}=\mathrm{OAc}, \mathrm{R}_{5}=\mathrm{OAc}, \mathrm{R}_{6}=\mathrm{Ac}$, $\mathrm{R}_{7}=\beta-\mathrm{CH}_{3}, \mathrm{R}_{8}=\mathrm{H}$

E. obtusifolia

Marco et al.

$$
\text { 426. } \mathrm{R}_{1}=\mathrm{OiBu}, \mathrm{R}_{2}=\mathrm{H}, \mathrm{R}_{3}=\mathrm{OMB}, \mathrm{R}_{4}=\mathrm{OAc} \text {, }
$$$$
\mathrm{R}_{5}=\mathrm{OAc}, \mathrm{R}_{6}=\mathrm{Ac}, \mathrm{R}_{7}=\beta-\mathrm{CH}_{3}, \mathrm{R}_{8}=\mathrm{H}
$$

427. $\mathrm{R}_{1}=\mathrm{ONic}, \mathrm{R}_{2}=\mathrm{H}, \mathrm{R}_{3}=\mathrm{OMB}, \mathrm{R}_{4}=\mathrm{OAc}$,

$\mathrm{R}_{5}=\mathrm{OAc}, \mathrm{R}_{6}=\mathrm{Ac}, \mathrm{R}_{7}=\beta-\mathrm{CH}_{3}, \mathrm{R}_{8}=\mathrm{H}$

428. $\mathrm{R}_{1}=\mathrm{OMB}, \mathrm{R}_{2}=\mathrm{H}, \mathrm{R}_{3}=\mathrm{OMB}, \mathrm{R}_{4}=\mathrm{OiBu}$,

$\mathrm{R}_{5}=\mathrm{OAc}, \mathrm{R}_{6}=\mathrm{Ac}, \mathrm{R}_{7}=\beta-\mathrm{CH}_{3}, \mathrm{R}_{8}=\mathrm{H}$

429. $\mathrm{R}_{1}=\mathrm{OAc}, \mathrm{R}_{2}=\mathrm{H}, \mathrm{R}_{3}=\mathrm{OMB}, \mathrm{R}_{4}=\mathrm{OiBu}$,

$\mathrm{R}_{5}=\mathrm{OAc}, \mathrm{R}_{6}=\mathrm{Ac}, \mathrm{R}_{7}=\beta-\mathrm{CH}_{3}, \mathrm{R}_{8}=\mathrm{H}$

430. $\mathrm{R}_{1}=\mathrm{OMB}, \mathrm{R}_{2}=\mathrm{MB}, \mathrm{R}_{3}=\mathrm{OH}, \mathrm{R}_{4}=\mathrm{OAc}$,

$\mathrm{R}_{5}=\mathrm{OBz}, \mathrm{R}_{6}=\mathrm{Ac}, \mathrm{R}_{7}=\beta-\mathrm{CH}_{3}, \mathrm{R}_{8}=\mathrm{H}$

431. $\mathrm{R}_{1}=\mathrm{OMB}, \mathrm{R}_{2}=\mathrm{MB}, \mathrm{R}_{3}=\mathrm{OH}, \mathrm{R}_{4}=\mathrm{OiBu}$,

$\mathrm{R}_{5}=\mathrm{OAc}, \mathrm{R}_{6}=\mathrm{Ac}, \mathrm{R}_{7}=\beta-\mathrm{CH}_{3}, \mathrm{R}_{8}=\mathrm{H}$

432. $\mathrm{R}_{1}=\mathrm{ONic}, \mathrm{R}_{2}=\mathrm{Ac}, \mathrm{R}_{3}=\mathrm{OiBu}, \mathrm{R}_{4}=\mathrm{OAc}$, $\mathrm{R}_{5}=\mathrm{OAc}, \mathrm{R}_{6}=\mathrm{Nic}, \mathrm{R}_{7}=\beta-\mathrm{CH}_{3}, \mathrm{R}_{8}=\mathrm{Ac}$

E. dendroides

Corea et al. (euphodendroidin $\mathrm{I}$ )

433. $\mathrm{R}_{1}=\mathrm{H}, \mathrm{R}_{2}=\mathrm{H}, \mathrm{R}_{3}=\mathrm{OMeBu}$,

$\mathrm{R}_{4}=\mathrm{OMeBu}, \mathrm{R}_{5}=\mathrm{OMeBu}, \mathrm{R}_{6}=\mathrm{H}, \mathrm{R}_{7}=\beta$ -

$\mathrm{CH}_{3}, \mathrm{R}_{8}=\mathrm{H}$

434. $\mathrm{R}_{1}=\mathrm{H}, \mathrm{R}_{2}=\mathrm{H}, \mathrm{R}_{3}=\mathrm{OiBu}, \mathrm{R}_{4}=\mathrm{OiBu}$,

$\mathrm{R}_{5}=\mathrm{OiBu}, \mathrm{R}_{6}=\mathrm{H}, \mathrm{R}_{7}=\beta-\mathrm{CH}_{3}, \mathrm{R}_{8}=\mathrm{H}$

435. $\mathrm{R}_{1}=\mathrm{H}, \mathrm{R}_{2}=\mathrm{H}, \mathrm{R}_{3}=\mathrm{OMeBu}$,

$\mathrm{R}_{4}=\mathrm{OMeBu}, \mathrm{R}_{5}=\mathrm{OiBu}, \mathrm{R}_{6}=\mathrm{H}, \mathrm{R}_{7}=\beta$ -

$\mathrm{CH}_{3}, \mathrm{R}_{8}=\mathrm{H}$

436. $\mathrm{R}_{1}=\mathrm{H}, \mathrm{R}_{2}=\mathrm{H}, \mathrm{R}_{3}=\mathrm{OiBu}, \mathrm{R}_{4}=\mathrm{OiBu}$,

$\mathrm{R}_{5}=\mathrm{OMeBu}, \mathrm{R}_{6}=\mathrm{H}, \mathrm{R}_{7}=\beta-\mathrm{CH}_{3}, \mathrm{R}_{8}=\mathrm{H}$

437. $\mathrm{R}_{1}=\mathrm{H}, \mathrm{R}_{2}=\mathrm{Ac}, \mathrm{R}_{3}=\mathrm{OH}, \mathrm{R}_{4}=\mathrm{OiBu}$,

$\mathrm{R}_{5}=\mathrm{OMeBu}, \mathrm{R}_{6}=\mathrm{H}, \mathrm{R}_{7}=\beta-\mathrm{CH}_{3}, \mathrm{R}_{8}=\mathrm{H}$

438. $\mathrm{R}_{1}=\mathrm{H}, \mathrm{R}_{2}=\mathrm{H}, \mathrm{R}_{3}=\mathrm{OiBu}, \mathrm{R}_{4}=\mathrm{OiBu}$,

$\mathrm{R}_{5}=\mathrm{OMeBu}, \mathrm{R}_{6}=\mathrm{H}, \mathrm{R}_{7}=\beta-\mathrm{CH}_{3}, \mathrm{R}_{8}=\mathrm{H}$

439. $\mathrm{R}_{1}=\mathrm{H}, \mathrm{R}_{2}=\mathrm{H}, \mathrm{R}_{3}=\mathrm{OMeBu}, \mathrm{R}_{4}=\mathrm{OiBu}$,

$\mathrm{R}_{5}=\mathrm{OMeBu}, \mathrm{R}_{6}=\mathrm{H}, \mathrm{R}_{7}=\beta-\mathrm{CH}_{3}, \mathrm{R}_{8}=\mathrm{H}$

440. $\mathrm{R}_{1}=\mathrm{OAc}, \mathrm{R}_{2}=\mathrm{Ac}, \mathrm{R}_{3}=\mathrm{OiBu}, \mathrm{R}_{4}=\mathrm{OiBu}$,

$\mathrm{R}_{5}=\mathrm{OAc}, \mathrm{R}_{6}=\mathrm{Ac}, \mathrm{R}_{7}=\beta-\mathrm{CH}_{3}, \mathrm{R}_{8}=\mathrm{H}$

441. $\mathrm{R}_{1}=\mathrm{OAc}, \mathrm{R}_{2}=\mathrm{Ac}, \mathrm{R}_{3}=\mathrm{OBz}, \mathrm{R}_{4}=\mathrm{OiBu}$,

$\mathrm{R}_{5}=\mathrm{OAc}, \mathrm{R}_{6}=\mathrm{Ac}, \mathrm{R}_{7}=\beta-\mathrm{CH}_{3}, \mathrm{R}_{8}=\mathrm{H}$

442. $\mathrm{R}_{1}=\mathrm{OAc}, \mathrm{R}_{2}=\mathrm{BzOCH}_{2} \mathrm{CO}, \mathrm{R}_{3}=\mathrm{OAc}$,

$\mathrm{R}_{4}=\mathrm{OiBu}, \mathrm{R}_{5}=\mathrm{OAc}, \mathrm{R}_{6}=\mathrm{Ac}, \mathrm{R}_{7}=\beta-\mathrm{CH}_{3}$,

$\mathrm{R}_{8}=\mathrm{H}$

443. $R_{1}=$ OAc, $R_{2}=A c, R_{3}=A c, R_{4}=O A c$,

$\mathrm{R}_{5}=\mathrm{H}, \mathrm{R}_{6}=\mathrm{Nic}, \mathrm{R}_{7}=\alpha-\mathrm{CH}_{3}, \mathrm{R}_{8}=\mathrm{Ac}$

Jakupovic et al. (1998b) 
Table 1 continued

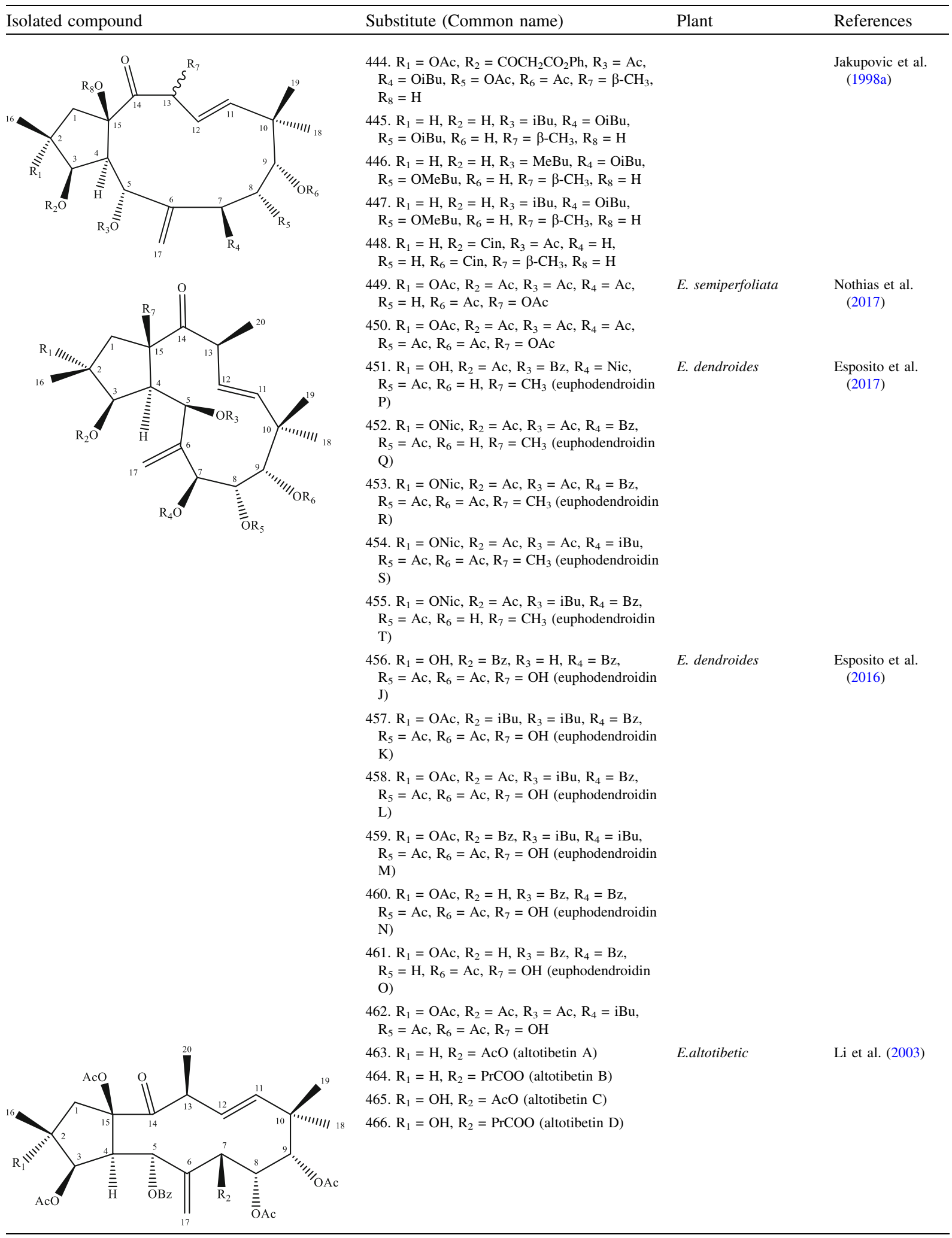


Table 1 continued

\begin{tabular}{|c|c|c|c|}
\hline Isolated compound & Substitute (Common name) & Plant & References \\
\hline & $\begin{aligned} \text { 467. } R_{1}=H, R_{2} & =B z, R_{3}=\alpha-A c, R_{4}=O A c \\
R_{5}=O A c, R_{6} & =H\end{aligned}$ & E. mongolica & $\begin{array}{l}\text { Hohmann et al. } \\
\text { (2003b) }\end{array}$ \\
\hline & $\begin{aligned} \text { 468. } \mathrm{R}_{1}=\mathrm{H}, \mathrm{R}_{2} & =\mathrm{Ac}, \mathrm{R}_{3}=\alpha-\mathrm{Ac}, \mathrm{R}_{4}=\mathrm{OBz} \\
\mathrm{R}_{5}=\mathrm{OAc}, \mathrm{R}_{6} & =\mathrm{Ac}\end{aligned}$ & E. turczaninowii & $\begin{array}{l}\text { Liu and Tan } \\
(2001)\end{array}$ \\
\hline & $\begin{aligned} \text { 469. } R_{1}=H, R_{2} & =A c, R_{3}=\alpha-A c, R_{4}=O A c \\
R_{5}=O A c, R_{6} & =A c\end{aligned}$ & & \\
\hline & $\begin{array}{l}\text { 470. } R_{1}=O A c, R_{2}=A c, R_{3}=\alpha-A c, R_{4}=O A c \\
R_{5}=H, R_{6}=A c \text { (esulatin } B \text { ) }\end{array}$ & E. esula & $\begin{array}{l}\text { Hohmann et al. } \\
\text { (1997) }\end{array}$ \\
\hline & $\begin{array}{l}\text { 471. } \mathrm{R}_{1}=\mathrm{H}, \mathrm{R}_{2}=\mathrm{Pr}, \mathrm{R}_{3}=\alpha-\mathrm{Bz}, \mathrm{R}_{4}=\mathrm{OiBu} \\
\mathrm{R}_{5}=\mathrm{OH}, \mathrm{R}_{6}=\mathrm{H} \text { (enukokurin) }\end{array}$ & E. lateriflora & $\begin{array}{l}\text { Fakunle et al. } \\
\text { (1989) }\end{array}$ \\
\hline & $\begin{array}{l}\text { 472. } \mathrm{R}_{1}=\mathrm{H}, \mathrm{R}_{2}=\mathrm{iBu}, \mathrm{R}_{3}=\beta-\mathrm{H}, \mathrm{R}_{4}=\mathrm{H} \\
\mathrm{R}_{5}=\mathrm{H}, \mathrm{R}_{6}=\mathrm{Ac}\end{array}$ & E. characias & $\begin{array}{l}\text { Seip and Hecker } \\
\text { (1984) }\end{array}$ \\
\hline & $\begin{array}{l}\text { 473. } \mathrm{R}_{1}=\mathrm{H}, \mathrm{R}_{2}=\mathrm{Tig}, \mathrm{R}_{3}=\beta-\mathrm{H}, \mathrm{R}_{4}=\mathrm{H} \\
\mathrm{R}_{5}=\mathrm{H}, \mathrm{R}_{6}=\mathrm{Ac}\end{array}$ & & \\
\hline & $\begin{array}{l}\text { 477. } R_{1}=O A c, R_{2}=A c, R_{3}=\beta-A c, R_{4}=H \\
\quad R_{5}=B z, R_{6}=N i c\end{array}$ & & \\
\hline & $\begin{array}{l}\text { 475. } R_{1}=O A c, R_{2}=A c, R_{3}=\beta-A c, R_{4}=H \\
R_{5}=\text { Tig, } R_{6}=N i c\end{array}$ & & \\
\hline & 476. & E. hyberna & $\begin{array}{l}\text { Ferreira et al. } \\
\quad(2002)\end{array}$ \\
\hline & $\begin{array}{l}\text { 477. } R_{1}=H, R_{2}=\beta-C_{3}, R_{3}=A c(\text { kansuinin } B) \\
\text { 478. } R_{1}=A c, R_{2}=\beta-C_{3}, R_{3}=H(\text { kansuinin } C)\end{array}$ & E. kansui & $\begin{array}{l}\text { Wang et al. } \\
\text { (2002) }\end{array}$ \\
\hline & 479. $R_{1}=A c, R_{2}=\alpha-C_{3}, R_{3}=H$ & E. esula & Liu et al. (2002) \\
\hline & $\begin{array}{l}\text { 480. } R_{1}=O H, R_{2}=B z, R_{3}=H, R_{4}=O A c \\
R_{5}=O B z, R_{6}=A c(\text { cyparissin } A)\end{array}$ & E. cyparissias & $\begin{array}{l}\text { Lanzotti et al. } \\
\text { (2015) }\end{array}$ \\
\hline & $\begin{array}{l}\text { 481. } \mathrm{R}_{1}=\mathrm{OH}, \mathrm{R}_{2}=\mathrm{Bz}, \mathrm{R}_{3}=\mathrm{Ac}, \mathrm{R}_{4}=\mathrm{OAc} \\
\mathrm{R}_{5}=\mathrm{OBz}, \mathrm{R}_{6}=\mathrm{H}(\text { cyparissin } \mathrm{B})\end{array}$ & & \\
\hline & $\begin{aligned} \text { 482. } \mathrm{R}_{1}=\mathrm{H}, \mathrm{R}_{2} & =\mathrm{Ac}, \mathrm{R}_{3}=\mathrm{Ac}, \mathrm{R}_{4}=\mathrm{Pr} \\
\mathrm{R}_{5}=\mathrm{OAc}, \mathrm{R}_{6} & =\mathrm{Ac}\end{aligned}$ & E. sororia & Lu et al. (2014) \\
\hline & $\begin{array}{l}\text { 483. } \mathrm{R}_{1}=\mathrm{ONic}, \mathrm{R}_{2}=\mathrm{iVal} \text { (isoValeryl), } \mathrm{R}_{3}=\mathrm{Ac} \\
\mathrm{R}_{4}=\mathrm{H}, \mathrm{R}_{5}=\mathrm{H}, \mathrm{R}_{6}=\mathrm{H} \text { (euphodendrophane } \\
\mathrm{G})\end{array}$ & E. dendroides & $\begin{array}{l}\text { Jadranin et al. } \\
\text { (2013) }\end{array}$ \\
\hline
\end{tabular}


Table 1 continued

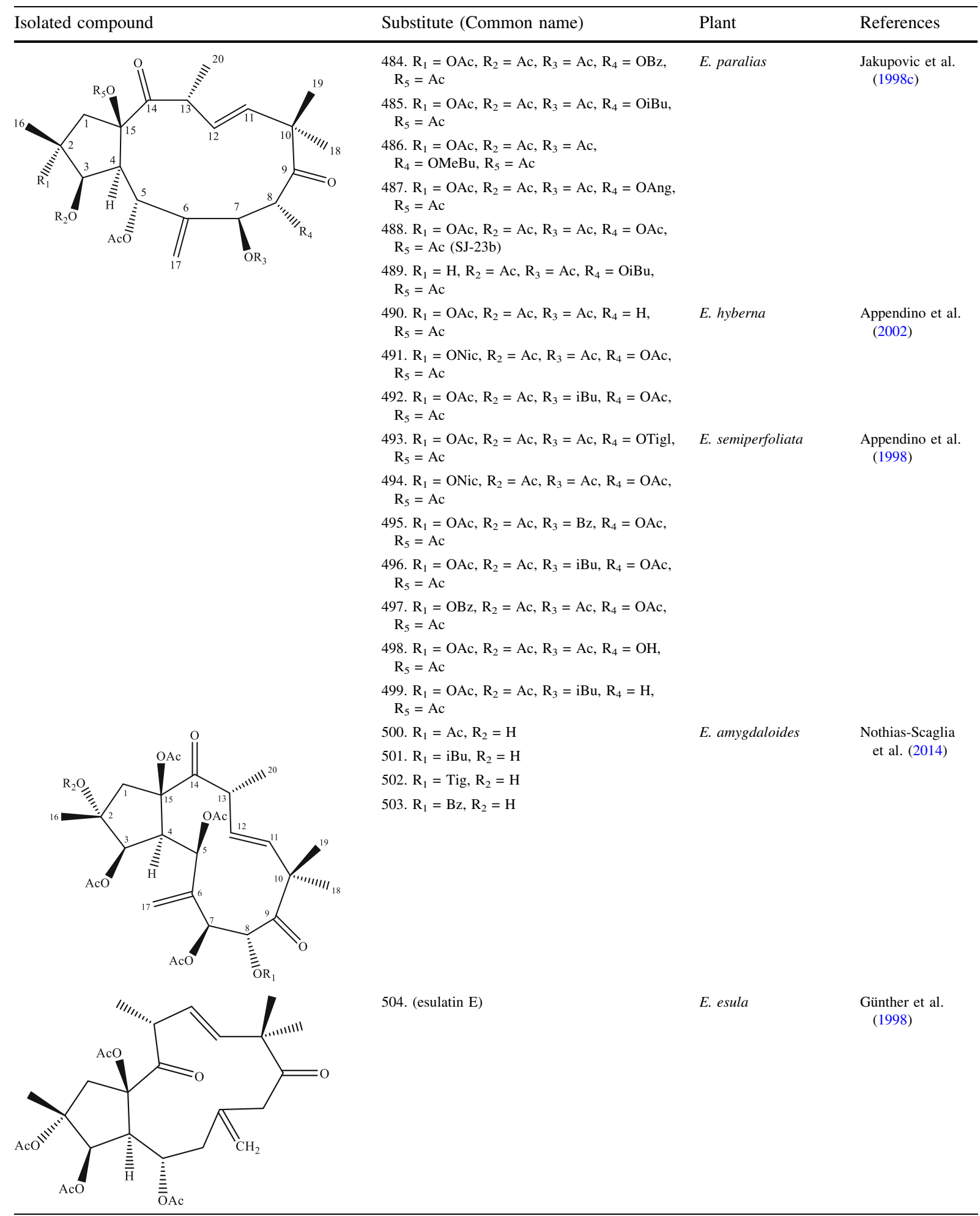


Table 1 continued

\begin{tabular}{|c|c|c|c|}
\hline Isolated compound & Substitute (Common name) & Plant & References \\
\hline & 505. (helioscopianoid M) & E. helioscopia & $\begin{array}{r}\text { Mai et al. } \\
\text { (2018b) }\end{array}$ \\
\hline
\end{tabular}

Andrea Vasas and coworkers also developed a three-step sample for the screening of macrocyclic diterpenes in 33 Euphorbiaceae species. The sample preparation includes the percolation of powdered plant material with $\mathrm{MeOH}$ at room temperature. After concentration, water is added to the extract and it is subjected to solvent-solvent partitioning with $\mathrm{CHCl}_{3}$. The organic phase is subjected to polyamide- 6 column chromatography with a $\mathrm{MeOH}: \mathrm{H}_{2} \mathrm{O}$ gradient system $(1: 3,3: 1,4: 1$ and $1: 0)$ as eluent. The concentrated fractions are monitored by TLC (Fig. 1), with the use of $\mathrm{CHCl}_{3}: \mathrm{Me}_{2} \mathrm{CO} \quad(19: 1)$ and cyclohexane:EtOAc:EtOH (60:30:1) as mobile phases. Spraying with concentrated sulfuric acid, followed by heating at $105{ }^{\circ} \mathrm{C}$, is used for visualization of the diterpene spots which are appeared in black or dark brown color or rarely blue spots with $\mathrm{Rf}$ values of $0.2-0.8$. The fraction eluted with $\mathrm{MeOH}(60 \%)$ are enriched in the diterpene esters, with a mixture of $\mathrm{MeOH}: \mathrm{H}_{2} \mathrm{O}$ (4:1) rich in triterpenes and fats, and fractions obtained from $\mathrm{MeOH}(100 \%)$ contained large amounts of chlorophyll (Vasas et al. 2012). For final purification, repeated column chromatography separations, using different adsorbents have been applied as is reported in supplementary file.

\section{Biological activities and SAR studies}

Antineoplastic activity

Liu and Tan (2001) evaluated five new (377-379, 467, and 468) along with one known 497 jatrophane diterpenoids from E. turczaninowii in mouse ear inflammation assay and in cytotoxicity test against the mouse melanoma B16 cell line. Results showed that all the six exhibited no irritant activity $\left(\mathrm{ID}_{50}^{24-}\right.$ $>100 \mu \mathrm{g} /$ ear) in a mouse ear inflammation model and also no significant cytotoxicity when evaluated against the B16 melanoma cell line ( $\mathrm{IC}_{50}>5 \mu \mathrm{g} / \mathrm{mL}$ ) (Liu and Tan 2001).

Liu et al. (2002) investigated the cytotoxicity of two new macrocyclic jatrophanes (91 and 479) of E. esula by the standard MTT test for the tumor cell lines mouse melanoma B16, human epidermoid KB, human hepatoma SMMC, human gastric adenocarcinoma BGC, and leukemia HL-60 (vinblastine was used as positive control with $\mathrm{IC}_{50}$ being $2.44,3.23,2.78,1.47$, and $1.32 \mu \mathrm{g} / \mathrm{mL}$, respectively). The results indicated that 479 was cytotoxic to $\mathrm{B} 16$ with $\mathrm{IC}_{50}=1.81 \mu \mathrm{g}$ / $\mathrm{mL}$. The irritant activity assay indicated that both 91 and 479 are inactive in a mouse ear inflammation model $\left(\mathrm{ID}_{502}^{4}>100 \mu \mathrm{g} / \mathrm{ear}\right.$ ) (Liu et al. 2002).

Wang et al. (2002) isolated three jatrophanes kansuinins A (95), B (477), and C (478) from $E$. kansui and tested their effects on the division of isolated cells from the early Xenopus laevis embryo to investigate the cell growth inhibition. The results showed that 95 and 478 did not inhibit cell division of the isolated cells. However, treatment of cells with 10 , 50 and $200 \mu \mathrm{g} / \mathrm{mL}$ kansuinin B (477) structure of which was very similar to that of 95 resulted in cleavage arrest in $57 \%, 87 \%$, and $98 \%$ of cells, respectively. Concerning observations, among these three jatrophanes $(95,477$, and 478$)$ only kansuinin B (477) showed remarkable activity, resulting in $87 \%$ cleavage arrest at $50 \mu \mathrm{g} / \mathrm{mL}$ (Wang et al. 2002).

Miglietta et al. (2003) isolated known jatrophanes (362, 358, and 357) from E. semiperfoliata. To discover more desirable biological analogues with 
mechanisms similar to that of paclitaxel, they focused on the action of these compounds on tubulin function, both in the assembly of purified tubulin and in living cells. These jatrophanes did not interfere with GTPinduced tubulin assembly in contrast to the other microtubule-interacting drugs; instead, they induced the formation of tubulin polymers rapidly in the absence of other promoters. Besides, jatrophane polymerization products were destabilized and disassembled by calcium ions unlike those of paclitaxel (Schiff and Horwitz 1980). In addition, no irregular tubulin polymerization products were formed. In this regard, jatrophanes interact with the tubulin differently from paclitaxel and their biological activity cannot be caused by suppression of microtubule dynamics, which is the target of many microtubuleinteracting agents. At a cellular level, jatrophanes reorganize microtubules without inducing microtubule bundling in contrast to the common tubulinpolymerizing agents. These results depicted that jatrophane polyesters from E. semiperfoliata can represent a new type of active tubulin-interacting pharmacophores (Miglietta et al. 2003).

Betancur-Galvis et al. (2003) evaluated the antitumor activity of seven macrocyclic jatrophanes (425-431) of E. obtusifolia by nicotinamide adenine dinucleotide factor (NADH) oxidase activity assay. The results depicted that all (425-431) inhibited $\mathrm{NADH}$ oxidase activity, with $\mathrm{IC}_{50}$ values ranging from $5.1 \pm 0.2 \mu \mathrm{M}$ for 371 to $13.9 \pm 1.8 \mu \mathrm{M}$ for 366 . The performing SAR studies showed that 344 , the strongest inhibitor, displayed an isobutyrate group at C-7 leading to an $\mathrm{IC}_{50}$ value of $6.3 \mu \mathrm{M}$. Less active compounds (426 and 427) had an acetoxy group at C-7. Even 429 with an isobutyrate group at C-7 and an acetoxy group at $\mathrm{C}-2$ showed a reduction in $\mathrm{NADH}$ oxidase inhibitory. Accordingly, it was proposed that the presence of acetoxy groups at C-2 and C-7 reduces the inhibitory effect on the NADH oxidase activity. The suggested mechanism was associated with the inhibition of the mitochondrial electron transport chain that arose from the breakdown of the transmembrane mitochondrial potential, resulting in early apoptosis (Betancur-Galvis et al. 2003).

Valente et al. (2003) evaluated pubescenes A (270), B (271), and C (271) of E. pubescens for their in vitro effect on the growth of three human cancer cell lines: MCF-7(breast), NCI-H460 (lung), and SF-268 (CNS) as well as their capacity to interfere with the proliferation of human peripheral blood lymphocytes. The compounds did not show any inhibitory activity on in vitro growth of the human cancer cell lines even at a concentration as high as $50 \mu \mathrm{M}$. They even had not any suppressor effects against the in vitro proliferation of human lymphocytes to phytohaemagglutinin even when tested at $100 \mu \mathrm{M}$ (Valente et al. 2003).

Valente et al. (2004a, b, c) evaluated jatrophanes euphopubescenol (122) and euphopubescene (300) of E. pubescens for their ability to inhibit the in vitro growth of three human tumor cell lines: MCF-7, NCIH460, and SF-268. They inhibited both MCF-7 and NCI-H460 cell lines with $\mathrm{GI}_{50}$ values ranging between $40.9 \mu \mathrm{M}$ and $95.3 \mu \mathrm{M}$ but were not effective on the SF-268 cell line (Valente et al. 2004b).

Valente et al. (2004a, b, c) isolated a new jatrophane diterpene, pubescenol (123) from $E$. pubescens evaluated for its ability to inhibit the in vitro growth of MCF-7, NCI-H460, and SF-268 cell lines. Results showed that 123 is a moderate growth inhibitor for all mentioned cell lines $\left(\mathrm{GI}_{50}\right.$ $=69.04 \pm 4.59,55.56 \pm 3.95$, and $75.16 \pm 6.54$, respectively) (Valente et al. 2004c).

$\mathrm{Lu}$ et al. (2008) isolated four new jatrophane-type diterpenoids $(3,162,164$ and 166) together with 16 known compounds from E. helioscopia. All the compounds were evaluated for their cytotoxicity against human cervical carcinoma cells (HeLa) and breast tumor cells (MDA-MB-231) among which only euphornin (208) was found to have inhibitory activity for the HeLa and MDA-MB-231 cells $\left(\mathrm{IC}_{50}=3.1\right.$ and 13.4 $\mu \mathrm{M}$, respectively). All other jatrophanes were inactive $\left(\mathrm{IC}_{50}>10 \mu \mathrm{M}\right)$ on both cell lines (Lu et al. 2008).

Hegazy et al. (2010) isolated two new jatrophanes guyonianins E (364) and F (21) along with a known jatrophane diterpene (362) from methylenechloride/ methanol extract of the aerial parts of E. guyoniana. Compound 362 showed significant activity $\left(\mathrm{IC}_{50-}\right.$ $=35 \mu \mathrm{M})$ while new compounds 364 and 21 had a moderate activity $\left(\mathrm{IC}_{50}=70\right.$ and $100 \mu \mathrm{M}$, respectively) against human embryonic kidney 293 (HEK293) cells (Hegazy et al. 2010).

Wang et al. (2012) isolated two jatrophanes, kansuinin A (95) and kansuinin B (477), by cytotoxic assay guided multistep separation on the dichloromethane extract of the roots of E. kansui. These diterpenoids were evaluated in vitro for their 
cytotoxicity effect in hepatoma cell lines (Bel-7402 and Bel-7402/5FU) and human gastric carcinoma cell lines (BGC-823 and SGC-7901) and displayed no antiproliferative effects (Wang et al. 2012).

Liu et al. (2014) isolated a new jatrophane-type diterpenoid (218) from the whole plant of E. lunulata Bge. The in vitro antiproliferative activities against MCF-7 and non-small cell lung carcinoma (NCIH460) cell lines for this compound were evaluated. The results showed moderate cytotoxic activities for both cell lines with the $\mathrm{IC}_{50}$ values ranging from 32.1 to $58.2 \mu \mathrm{M}$ (Liu et al. 2014).

Ghanadian et al. (2015) isolated three new diterpenes (342-344) from E. osyridea and analyzed their cytotoxicity by performing MTT, annexin V-FITC, and PI staining assays against Caov-4 and OVCAR-3 ovarian cancer cell lines. The results showed that 131-133 inhibit cell proliferation through apoptosis in both Caov-4 and OVCAR-3 cells. Compounds 342 and 343 illustrated more significant inhibitory effects with $\mathrm{IC}_{50}$ values of $38.81 \pm 3.30$ and $42.59 \pm 4.50 \mu \mathrm{M}$ on the OVCAR-3 cell line, and $46.27 \pm 3.86$ and $36.48 \pm 3.18 \mu \mathrm{M}$ on the Caov- 4 cell line. Compound 344 showed moderate cytotoxicity with $\mathrm{IC}_{50}$ values of $75.65 \pm 2.56$ and $85.86 \pm 6.75 \mu \mathrm{M}$ against OVCAR-3, and Caov-4 cell lines, respectively. Doxorubicin as the standard drug suppressed the ovarian cancer cells, with $\mathrm{IC}_{50}$ values of $0.33 \pm 0.09$ and $0.84 \pm 0.19$ on OVCAR-3 and Caov-4 cells, respectively (Ghanadian et al. 2015).

Shadi et al. (2015) isolated jatrophane 296 from $E$. connata and evaluated its cytotoxicity using MTT assay against two MCF-7 and MDA-MB 469 human breast cancer cell lines. It showed weak cytotoxicity with $\mathrm{IC}_{50}$ values of $55.67 \pm 7.09 \mu \mathrm{M}$ against MDA$\mathrm{MB}$ and moderate cytotoxicity with $\mathrm{IC}_{50}$ values of $24.33 \pm 3.21 \mu \mathrm{M}$ against MCF-7 cell line (Shadi et al. 2015).

Bahmani et al. (2017) evaluated cytotoxicity and the molecular mechanism of apoptosis induced by the novel 'jatropha-6(17),11E-diene' class derivatives (342-344) previously extracted from E. osyridea on Caov-4 and OVCAR-3 ovarian cancer cell lines. 133 showed the lowest activity against Caov-4 and OVCAR-3 ovarian cell lines $\left(\mathrm{IC}_{50}=85.86 \pm 6.75\right.$ and $75.65 \pm 2.56 \mu \mathrm{M}$, respectively). 343 showed stronger cytotoxic effects $\left(\mathrm{IC}_{50}=36.48 \pm 3.18\right.$ and $42.59 \pm 4.50 \mu \mathrm{M})$ than $133\left(\mathrm{IC}_{50}=46.27 \pm 3.86\right.$ and $38.81 \pm 3.30 \mu \mathrm{M})$ upon which it seems that benzoyl moiety occupying position 3 and C-8 occupation with propyl group in Euph B have critical effects in the potency of this jatrophane (Pešić et al. 2011). Apoptosis evaluation showed 342-344 increase induction of both early and late apoptosis $(\mathrm{P}<0.01)$. Mitochondrial membrane potential $(\Delta \Psi \mathrm{m})$, ROS production, and caspase 3 and 9 activation were also evaluated which were all increased by these compounds in treated cells. According to these observations, 342 and 343 displayed significant inhibitory effects on OVCAR-3 and Caov-4 proliferation and induction of apoptosis. Induced ROS production in Caov-4 and OVCAR-3 was evaluated 2.6 and 4.4 for 131 ; and 4.7 and 9.9 fold/control for 343 , respectively. In this regard, ROS overproduction and trigger of caspase activation might be the potential mechanism of these compounds interposing apoptosis in the ovarian cancer cells by mitochondria or pro-oxidant activity of ionizable groups of 342 and 343 (Bahmani et al. 2017).

MDR reversing activity

In chemotherapy, P-gp is a membrane protein that confers upon cells the ability to resist lethal doses of certain cytotoxic drugs by pumping them out of the cells leading to a reduction of their cytotoxic or antiproliferation effects (Barile et al. 2008b). The presence of P-gp transport proteins in the microorganism membrane makes also challenge the treatment of the infectious diseases, as they cause a mechanism of multidrug resistance (MDR) developed during treatment, by pumping out anti-infectious drugs (Schnabel and Hiersemann 2009; Schnabel et al. 2010; Shukla et al. 1999; Sutherland and Polley 2011). The emergence of cancer MDR has been pointed out as one of the major barriers to successful chemotherapy. The most well-known mode of resistance has been associated with P-gp (ATB-binding cassette sub-family B member 1 (ABCB1)/P-gp), the first human $\mathrm{ABC}$ transporter to be described. The overexpression of $\mathrm{ABCB} 1$ results in reduced intracellular concentration of drugs to levels leading to treatment failure, causing also cross-resistance or cross-sensitivity to other drugs (Gottesman et al. 2002). To enhance the efficacy of chemotherapy, several approaches have been proposed to circumvent MDR. Developing the molecules that are able to impair the drug efflux mediated by $\mathrm{ABCB} 1$ as well as the development of collateral 

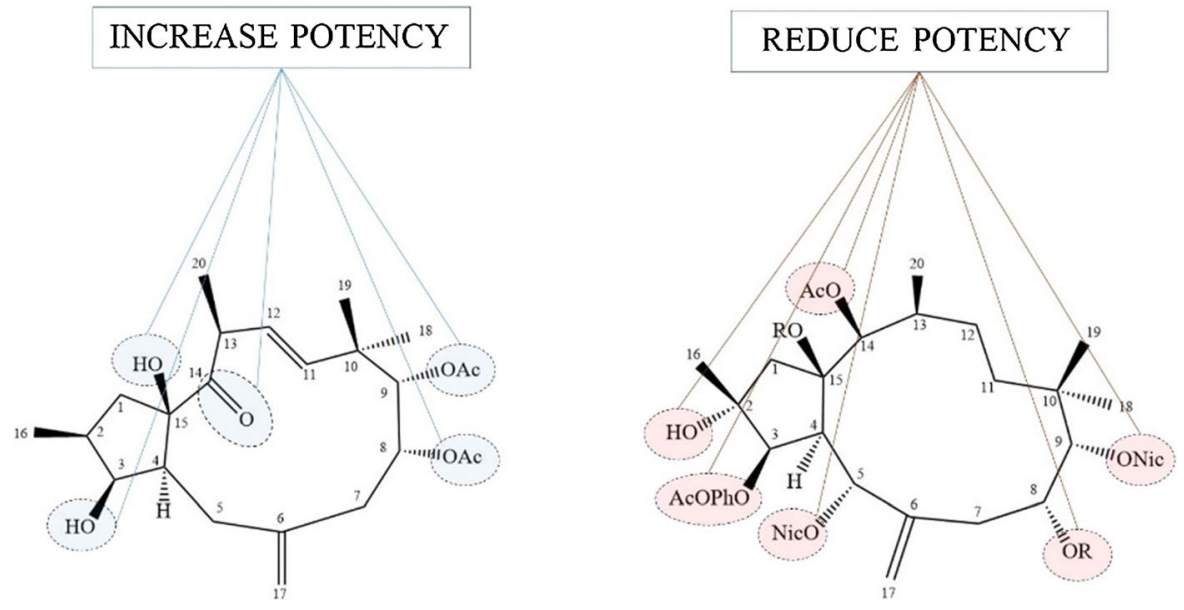

Fig. 13 Key pharmacophoric elements for the anti-MDR activity of P-gp

sensitivity agents lay among the most promising strategies (Callaghan et al. 2014; Szakács et al. 2014). Therefore, potent and selective P-gp inhibitors are potential targeted agents to combat chemotherapy drug resistance. In drug discovery programs for cancer MDR, a large number of compounds have been investigated (Eid et al. 2015; Palmeira et al. 2012; $\mathrm{Wu}$ et al. 2011) among which the polyoxygenated jatrophane and lathyrane-type macrocyclic diterpenes from Euphorbia species have shown potential antiMDR activities, by ABCB1 modulation and/or by selective targeting of MDR cancer cells (Corea et al. 2009; Vasas and Hohmann 2014; Vieira et al. 2014).

Ferreira et al. (2014) in a review article provided a summary (2001-2013) of anticancer compounds from Euphorbia and Momordica species comprising diterpenes, triterpenes, and phenolic derivatives, particularly for their P-gp inhibition ability (Ferreira et al. 2014). In another study, Amaral et al. (2016) prepared a mini-review focusing on the property of MDR cancer cells (proliferation, apoptotic mechanism, efflux pumps) affected by bioactive compounds.

A set of over seventy jatrophanes and modified jatrophanes have been specifically investigated by Corea et al. (2009) for their MDR reversing potential (Corea et al. 2009). This wide analysis let the authors attribute the MDR reversal activity of these compounds to the key pharmacophoric elements as follows (Fig. 13):

Hohmann and others have isolated 22 jatrophane polyesters from Euphorbia genera reported in several articles over the period of 2001 to 2003, three of which $(375,376$, and 467) were from E. mongolica; 168,199 , 200, 301, 302, and 305 from E. serrulata; 374 (esulatin A), 382 (esulatin D), 470 (esulatin B) from E. esula; 381, 351, 354, 352, and 353 from E. peplus (Hohmann et al. 2002); a novel diterpene polyester named euphosalicin (383) and finally two new jatrophanes (26 and 372) from E. salicifolia (Hohmann et al. 2001a). These compounds were investigated for the reversal of MDR in L5178 mouse lymphoma cells using the rhodamine 123 (Rho123) exclusion test. 375, 376, and 467 displayed a significant effect on inhibiting the efflux-pump activity of multidrug-resistant L5178 mouse lymphoma cells as compared with that of the positive control 'verapamil' (FAR $=13.14$ at $23 \mu \mathrm{M}$ ) in the range of $11.2-112 \mu \mathrm{M}$ as expressed by the FAR increasing at higher concentrations (FAR = $12.29,2.60,2.79$ at $11.2 \mu \mathrm{M}$ and $\mathrm{FAR}=22.92$, 18.02, 29.29 at $112 \mu \mathrm{M}$ ) (Hohmann et al. 2003b). 381, 354 , and 352 also displayed strong activity (FAR = 71.98-78.88) compared with that of the positive control 'verapamil' (FAR $=8.27$ ); while 374,382 , 470 , and 372 revealed weak potency. 302 and 353 had low effect on the drug accumulation at higher concentrations than at lower ones; in these cases, the increased membrane permeability could be responsible for the toxic effect that resulted in enhanced Rho123 diffusion out of the treated cells due to a membrane disintegration (Hohmann et al. 2002). The novel diterpene polyester 383 displayed considerable potency in inhibiting the efflux-pump activity of MDR 
P-gp in mouse lymphoma cells (FAR $=22.46$ at $2 \mu \mathrm{g} /$ $\mathrm{mL}$ ) being even stronger than the positive control 'verapamil' (FAR $=8.49$ at $2 \mu \mathrm{g} / \mathrm{mL}$ ) (Hohmann et al. 2001a). Comparison of the pairs (302 and 305), (374 and 372), and (354 and 352) differing only in the lipophilicity of one of the substituents $(\mathrm{OH}, \mathrm{OAc}$, $\mathrm{OiBu}$ ) demonstrated an increase in the MDR modifier effect. These data supported the conclusion that the effect on drug accumulation in drug-resistant cells is proportional to the hydrophobicity. Surprisingly, other structurally related pairs of compounds such as (374 and 381), with 2-OAc and 2-H and with 9-OAc and 9-ONic substitutions, respectively, and (351 and 353) differing only in the esterification at C-7-C-9 (7-OAc/ 7-OiBu, 8-OAc/8-OH, and 9-OAc/9-ONic) exerted very different effects in the modulation of the MDR of mouse lymphoma cells (Hohmann et al. 2002). This observation has been explained by the high flexibility of the macrocyclic ring of the jatrophane skeleton (Appendino et al. 1998).

Corea et al. isolated ten closely related jatrophanes from $E$. dendroides, nine of which were new, euphodendroidins A-I (417-424, and 432) and one was known (444) (Corea et al. 2003a). In another research Corea et al. isolated five new jatrophanes (pepluanins A-E (181-183, 415, and 416)) together with two known analogues (355 and 443) from E. peplus (Corea et al. 2004a) and twelve new diterpenes named euphocharacins A-L (401-412) from E. characias (Corea et al. 2004b). The inhibitory activity of all compounds was assayed in Pgp-mediated daunomycin transport efflux. Euphodendroidin D (420) (Corea et al. 2003a) and pepluanin A (181) (Corea et al. 2004a) together with euphocharacins C (403) and I (409) was found to be highly potent inhibitors since they were almost twofold more efficient than 'cyclosporin A', the golden standard of P-gp modulators. Thus, the following sequence in efficiency at C-3 may now be proposed as: propionyl $>$ benzoyl $>$ acetyl, isobutyryl. Another positive role was played by the benzoyl at C-9 (euphocharacin C (403), 123\%) which was better than nicotinyl in euphocharacin B (402) (72\%). However, they went beyond the southwestern fragment of the molecule (C-2/C-5) binding by performing SAR studies on pepluanins A-E (181-183, 415, and 416) and underlined the importance of the substitution on other carbons of the medium-sized ring C-8, C-9, C14, and C-15 in modulating the activity. Observations showed this series of seven jatrophane diterpenes (pepluanins A-E, 355, and 443) highlighting the importance of an acetoxyl at C-8 (by comparison to a free hydroxyl), and of a free hydroxyl at C-15. Moreover, a carbonyl at C-14 and acetoxyl at C-9 were also favorable substitutions.

Corea et al. (2003a, b) isolated 10 terracinolides from $E$. dendroides, four of which (terracinolides J-L (32-34) and $13 \alpha-\mathrm{OH}$ terracinolide F (35)) were novel and two other, (abeodendroidin F (102) and epiabeodendroidin F (103)) were new (Corea et al. 2003b). The inhibitory effect of P-gp mediated daunomycin efflux by these compounds was evaluated relative to cyclosporine $\mathrm{A}$ (CsA) by monitoring intracellular drug accumulation. Terracinolide $\mathrm{H}$ (45) displayed significant inhibition, even more potent $(138 \pm 27 \%)$ than cyclosporin A (CsA). SAR studies demonstrated that the revertant activity of terracinolides and abeojatrophanes was strongly affected by the presence of a free hydroxyl group, with the following ranking of position: $3>15>13>2$ (Corea et al. 2003b).

Valente et al. (2004) isolated pubescenes A-D (270-272, and 297) from E. pubescens and evaluated them for MDR reversing activity on L5178 mouse lymphoma cells. Tested compounds displayed strong activity in the cells by inhibiting the efflux-pump activity mediated by P-gp. Among all, pubescene A (270) $(\mathrm{FAR}=79.78$ in $32 \mu \mathrm{M})$ and D (297) (FAR = 111.00 in $32 \mu \mathrm{M})$ exhibited the highest effects in reversing MDR compared with the positive control 'verapamil'. The highest lipophilicity of pubescene A (270) due to the presence of four ester groups can be suggested for its strong activity. Another important structural feature was the presence of the benzoyl group as a sterically expansive group at C-7. Moreover, the higher activity of pubescene D (297) compared with 272 was due to a different configuration of the stereocenter at C-2 bearing an $\alpha$-oriented Methyl-16 (Valente et al. 2004a).

Ferreira et al. (2005) isolated rearranged jatrophanes (270-272, 297, 122, 300, and 123) from $E$. pubescens. They evaluated the ability of pubescene A (270), pubescene B (271), pubescene C (272), and pubescene D (297) as MDR modulators on L5178 mouse lymphoma cells, most of which were able to enhance the Rho123 accumulation of human MDR1gene-transfected mouse lymphoma cells. Euphopubescenol (122), euphopubescene (300), and pubescenol (123) were examined for the reversal of MDR 
on the human breast cancer MDA-MB-231(HTB-26) cell line by flow cytometry. The tested compounds did not show significant toxicity $(\mathrm{FAR}=0.9,0.8 ; 0.9,0.7$; $1.0,0.7$ at 5 and $20 \mu \mathrm{M}$, respectively) on MDA-MB231 cells since their $\mathrm{ID}_{50}$ values were higher than those of the DMSO control $(\mathrm{FAR}=0.8)$. Moreover, they were tested on MRP; carboxyfluorescein (BCECF-AM) served as a substrate for MRP-mediated drug efflux and its accumulation in the MDAMB-231 breast cancer cells was measured at 5 and $20 \mu \mathrm{M}$ respectively; Some compounds i.e. 270, 297 , 122, 300, and 123 showed a remarkable MRP-specific increase in fluorescence activity $(11.5,3.9,12.7,4.4$, and 5.8 at $20 \mu \mathrm{M})$ comparing to the positive control 'indomethacine' $($ FAR $=1.5$ at $27.9 \mu \mathrm{M}) \quad($ FERREIRA et al. 2005).

Buey et al. (2005) evaluated the interactions of microtubules with a number of compounds consist of jatrophanes described as stabilizing agents, to understand which ones have the capability to stabilize microtubules and mimic the activity of paclitaxel/docetaxel. Most of them including lonafarnib, dicumarol, lutein, and jatrophanes did not show any stabilizing effect on microtubules. Jatrophanes 362, 358, and 357 have not able to induce assembly at concentrations as high as $60 \mu \mathrm{M}$ guanosine triphosphate (GTP)-tubulin and $66 \mu \mathrm{M}$ ligand, as checked by centrifugation and electron microscopy. Overall, jatrophanes indicated no ability of induction or modulation in vitro microtubule assembly or displacement of a fluorescent taxoid (Flutax-2) from its binding site, suggesting that the microtubule-stabilizing activity of these compounds, if any, arises from interactions with other factors regulating cellular microtubule polymer mass rather than by direct binding to microtubules (Buey et al. 2005).

Engi et al. (2007) isolated nine diterpenes from $E$. esula (compounds 374 and 470), E. peplus (compounds 351 and 352), and E. serrulata (compounds 305, 302, and 168). Their MDR-reversal effects on a human colon (COLO320) cancer cell line, as well as the synergistic capacity of these compounds, were investigated. 305, 302, and 168 were found to be very strong inhibitors (FAR $>2.00$ at $40 \mu \mathrm{g} / \mathrm{mL}$ ). For 305 the effect was almost the same at the two concentrations $(\mathrm{FAR}=2.05$ at $4 \mu \mathrm{g} / \mathrm{mL}$ and $\mathrm{FAR}=2.03$ at $40 \mu \mathrm{g} / \mathrm{mL}$ ), meaning that both of the applied concentrations were in the saturation zone. $374,470,351$, and 352 were moderately effective $(0.59<$ FAR $<1.7)$.
Moreover, the synergistic capacity of these compounds in combination with 'epirubicin' was examined and 302 proved to be the most active, exhibiting a synergistic interaction (FIX $=0.25$ ) with 'epirubicin'. In contrast, 352 and 168 did not enhance the antiproliferative effect of the anticancer drug when applied in combination with the COLO320 cell line. Comparing the efficacies of 305 and 302, it can be presumed that the presence of a hydroxy group instead of peracylation is favourable as it concerns the antiproliferative activity in combination with 'epirubicin' (Engi et al. 2007).

Barile et al. (2008a, b) isolated new jatrophanes: euphoscopin M (104) and euphoscopin N (105) together with three other known analogues: euphoscopin C (245), euphornin (208), and epieuphoscopin B (254) from E. helioscopia. The biological activities of 104, 105, 245, 208, and 254 were monitored through their ability to inhibit P-gp-mediated mitoxantrone efflux leading to drug accumulation, measured by flow cytometry. All tested compounds exhibited concentration-dependent inhibition of mitoxantrone efflux. The concentration dependence analysis indicated that 254 with $\mathrm{IC}_{50}$ value of $1.71 \pm 0.83 \mu \mathrm{M}$ is twice as potent as the reference inhibitor 'cyclosporin $\mathrm{A}^{\prime}\left(\mathrm{IC}_{50}: 3.37 \pm 1.39 \mu \mathrm{M}\right)$. In contrast, 208 is much less efficient with $\mathrm{IC}_{50}$ value of $8.46 \pm 3.51 \mu \mathrm{M}$. Finally, the remaining compounds 104,105 , and 245 with $\mathrm{IC}_{50}$ values of $3.78 \pm 2.18,3.47 \pm 1.88$, and $3.58 \pm 1.78 \mu \mathrm{M}$, respectively appeared similar in activity to 'cyclosporin A' (Barile et al. 2008a). Comparing jatrophanes of E. helioscopia with those from other Euphorbia species (Corea et al. 2003a, b, 2004a, b), three main structure-activity relationships was deduced: (1) a marked, fivefold positive effect on P-gp inhibition played by a carbonyl versus an OAc group at position 9 when comparing 254 and 208; (2) a twofold positive effect of an OAc versus an $\mathrm{OBz}$ substituent at position 7 when comparing 254 and 245; (3) a neutral effect of having the double bond at either 11-12 or 12-13 positions in 105 and 104 (Barile et al. 2008a).

Duarte et al. (2008) isolated tuckeyanols A (143), B (144), and euphotuckeyanol (142) from E. tuckeyana. They tested them for P-gp modulating properties on human MDR1 gene-transfected and parental L5178 mouse lymphoma cell lines. Moreover, their combinations with the cytostatic anticancer drug 'epirubicine' were tested in order to obtain evidence as to 
additive or synergistic interactions. Tuckeyanols A (143), B (144), and euphotuckeyanol (142) showed strong activity $(\mathrm{FAR}=39.8,25.0$, and 81.0 at $4 \mu \mathrm{M}$, respectively) compared to 'verapamil' (FAR $=13.7$ at $10 \mu \mathrm{M})$. SAR studies on euphotuckeyanol (142) with the highest activity (FAR $=81.0$ at $4.0 \mu \mathrm{g} / \mathrm{mL}$ ) showed that 142 with seven ester residues has the highest values of $\log P(6.7)$, molecular weight (818), and the highest number of hydrogen bond acceptor groups (15 H-bond acceptors), all of which considered by several authors, as important requirements to P-gp modulation (Robert and Jarry 2003; Wiese and Pajeva 2001). Based on the spatial orientation of the SP2 terminal methylene group at C-6, analouge 142 showed endo-type conformation (NOESY correlation of exo-methylen $\mathrm{H}-17$ with 5-H ( $\beta$ )) (Jakupovic et al. 1998b, c; Marco et al. 1998) versus tuckeyanols A (143) and B (144) with exo-type conformation (Duarte et al. 2008) which may also be an important factor in MDR modulation. Concerning all these factors, it is difficult to explain which of them has the most relevant role for the high MDR-reversal activity. Duarte et al. observed that tested compounds exhibited a synergistic interaction with 'epirubicine' on the studied cell line (fractional inhibitory index (FIX) $=0.07-0.25$ ) among which, the most effective compound was euphotuckeyanol (142), expressing a low FIX (0.07 and 0.08 , respectively) in the checkerboard experiments (Duarte et al. 2008).

Pešic et al. (2011) investigated the inhibitory effect of two previously isolated jatrophanes from $E$. dendroides: euphodendrophane A (396) and euphodendrophane B (397) on the growth of the sensitive non-small cell lung carcinoma (NSCLC) cell line (NCI-H460) and its resistant counterpart (NCI-H460/ R). They further examined the potential of Euph A and B on mdr1 mRNA expression (Pešić et al. 2011). Both jatrophanes were more efficacious at P-gp inhibition than 'verapamil'. The development of synthetic jatrophanes based on their natural skeleton revealed that the presence of a lipophilic aromatic substituent at C-3 enhances the P-gp inhibitory activity compared to that of 'verapamil' (Schnabel et al. 2010). Although Euph $\mathrm{A}$ and $\mathrm{B}$ possess the smaller benzoyl residue at C-3, it does not influence their effect on P-gp inhibition which even overcomes the effect of 'verapamil'. Earlier findings highlighted the positive role of the free hydroxyl group at C-5 and acetyl group at C-8 being present in Euph A and B (Corea et al. 2009). Both jatrophanes significantly reduced the level of mdr1 expression in (NCI-H460) sensitive cells, suggesting that they could not induce the development of resistance in spite of PTX which is a P-gp substrate. For the resistant cells, PTX decreased the expression of mdr1, while both jatrophanes did not significantly influence the expression level. Observed inhibitory effect of Euph A and B on P-gp synthesis in sensitive cell line and P-gp activity in resistant cell lines could be considered as their application as adjuvant therapy in both sensitive and resistant malignancies. Pešic et al. had shown earlier that the resistant NCIH460/R cell line displays cross-resistance to paclitaxel, vinblastine, doxorubicin, epirubicin, and etoposide (Pesic et al. 2006) so they were interested in the investigation of the simultaneous combinations of Euph A/B and PTX on the MDR cancer cell lines. Importantly in this study, both Euph A and B enhanced the growth inhibition of PTX in a concentration-dependent manner. In this regard, all combinations used in the course of treatments of resistant NCIH460/R cells induced a strong synergistic effect. This research demonstrated that Euph A and B have the potential to reverse PTX resistance. Moreover, it was showed that the synergism between Euph A/B and PTX is partly due to their mutual effect on microtubule assembly (Pešić et al. 2011).

Vasas et al. (2011) isolated esulatins A-E (374, 470, 92, 382, and 504) and H-M (99, 128, 129, and 132-134) from E. esula. They were evaluated for their antiproliferative activity against a set of human adherent cell lines of gynecological origin (HeLa (cervix adenocarcinoma), Ishikawa (endometrial adenocarcinoma), and MCF-7 (breast epithelial adenocarcinoma)) using the MTT test and 'cisplatin' as positive control (Vasas et al. 2011). Moreover, Vasas et al. tested MDR-reversing activity of the compounds on L5178 mouse lymphoma cells, using a standard functional assay with Rho123. It was investigated that esulatins J (129), A (374), and E (504) were the most effective compounds against all cell lines; especially esulatin J (129) exhibited high cell growth inhibitory activity on Ishikawa $(98.4 \%$ at $30 \mu \mathrm{g} / \mathrm{mL})$ and MCF7 $(81.4 \%$ at $30 \mu \mathrm{g} / \mathrm{mL})$ cells. Esulatin I (128) and esulatin B (470) displayed marked inhibitory effects on MCF7 $(60.1 \%$ and $43.3 \%$ at $30 \mu \mathrm{g} / \mathrm{mL})$. SAR studies demonstrated that the most potent compounds, esulatins I, J, B and E (128, 129, 470, and 504) are tetra- or penta- esters of jatrophane polyols, which 
contain a keto group at C-9. Moreover, esulatin A (374), containing an epoxy group at C-11-C-12, found also to be effective against all three cell lines. All tested compounds differed significantly in the inhibition of the efflux pump activity of P-gp in tumor cells. Within the compounds investigated, esulatin J (129) $(\mathrm{FAR}=52.5$ at $40 \mu \mathrm{g} / \mathrm{mL})$ and esulatin $\mathrm{M}$ (134) $(F A R=119.9$ at $40 \mu \mathrm{g} / \mathrm{mL}$ ) were found to be the most powerful inhibitors of efflux pump activity. Their efficacy was 25-fold higher than that of positive control 'verapamil' (FAR $=23.2$ at $10 \mu \mathrm{g} / \mathrm{mL}$ ); thus, both 185 and 188 appeared to be promising leads for drug development to overcome the MDR of cancer cells (Vasas et al. 2011).

Aljancic et al. (2011) investigated the sensitivity of NCI-H460/R cells to another anticancer chemotherapeutic agent, doxorubicin, in the presence of six new jatrophanes, euphodendrophanes A-F (396-400, and 237) from $E$. dendroides. Moreover, the synergistic effect between these jatrophanes and the 'paclitaxel' was reported for the first time. They also investigated the effects of 396 and 397 on Rho123 accumulation in NCI-H460/R cells and compared the results with that of untreated resistant NCI-H460/R cells by the FAR. Rho123 accumulation was about twofold higher in untreated NCI-H460 cells compared to NCI-H460/R cells. A significantly higher accumulation of Rho123 in the NCI-H460/R cell line was obtained with 396 and 397 , compared to that of 'verapamil'. This observation had been elucidated by the positive role of certain pharmacophoric elements in the activities of jatrophanes against P-gp (Corea et al. 2009), like a free hydroxy group at $\mathrm{C}-5$ or an acetate group at $\mathrm{C}-8$, which are both present in 396 and 397. NCI-H460/R cells were exposed to combinations of $1,2.5$, and $5 \mu \mathrm{M}$ of 396 and 397 with 0.05-5 $\mu \mathrm{M}$ doxorubicin and paclitaxel and sensitivity were assessed using an SRB assay. The $\mathrm{IC}_{50}$ value for paclitaxel decreased in combination with 396, demonstrating 3-, 19-, and 38 -fold reversal activity for the aforementioned concentrations, respectively. An even more considerable effect was also obtained for 397, exhibiting 11-, 25-, and 60 -fold reversal activity. There were no significant differences in reversal activity at concentration levels of 2.5 and $5 \mu \mathrm{M}$ between 396 and 397 and 'verapamil'. Both jatrophanes at $5 \mu \mathrm{M}$ decreased the $\mathrm{IC}_{50}$ values of doxorubicin significantly, showing a similar reversal potential to 'verapamil'. These results pointed to the potential of 396 and 397 to reverse paclitaxel and doxorubicin resistance in the MDR cancer cell line used (Aljancic et al. 2011).

Valente et al. (2012) isolated three new jatrophanes euphomelliferine (293), euphomelliferenes A (294) and B (295) along with two known jatrophanes 306 and 302 from E. mellifera. 293-295 and 302 were investigated for their P-gp modulating effects on human MDR1-gene transfected mouse lymphoma cells (L5178Y MDR) and on human colon adenocarcinoma cells (COLO320) using 'verapamil' as a positive control. These compounds were also evaluated for their activity as apoptosis inducers using the annexinV/propidium iodide assay. 294 showed the highest P-gp modulating activity on both cell lines (FAR $=23.1$ and 5.5 at $20 \mu \mathrm{M}$ on L5178Y MDR and COLO320, respectively). But a much lower activity was observed in $295(\mathrm{FAR}=1.6$ and 2.8 at $20 \mu \mathrm{M})$ having an $\mathrm{OH}$ group at $\mathrm{C}-15$. However, when comparing the effects of 293 (FAR $=12.1$ and 5.1 at $20 \mu \mathrm{M})$ and 295 differing in the type of function at C-14, the presence of a carbonyl group at this position improves the activity, as for 293. The different location of one of the double bonds and the substitution at C-6 also influenced the efflux pump activity, as demonstrated by the FAR values of 293 and 302 $(\mathrm{FAR}=10.1$ and 3 at $20 \mu \mathrm{M})$ on the two cell lines. Contrarily, the configuration at C-2 did not seem to play a significant role in MDR modulatory activity (Valente et al. 2004a). It was concluded that the differences in the observed modulating effects between the two MDR cell lines may be associated with different levels of P-gp expression, which were lower in COLO320 cells according to immunohistological studies (Engi et al. 2006). Moreover, none of the tested compounds were able to induce significant apoptosis and cell death (Valente et al. 2012).

Rédei et al. (2012) isolated four novel (124-127) and one known (326) diterpenes from E. mongolica being evaluated for MDR reversing activity against human MDR gene-transfected L5178 mouse lymphoma cells via the intracellular accumulation of Rho123. Tested compounds displayed a significant inhibitory effect compared to 'verapamil'. SAR studies demonstrated that the differences in the substitution at positions C-7 and C-8 influences the ability to enhance intracellular drug accumulation by comparison of the structures 124-127 and the MDR-modifying activity $(\mathrm{FAR}=6.23,16.36,66.97$ and 37.12 at $2 \mu \mathrm{g} / \mathrm{mL}$ respectively). The MDR-modifying activity 
exhibited a definite increase with the size of the acyl group at $\mathrm{C}-7$ in the following sequence: acetyl $<$ propanoyl $<$ n-butanoyl $<$ isobutanoyl. 484 unsubstituted at C-7 and C-8, had a potency similar to that of $170(\mathrm{FAR}=6.3$ at $2 \mu \mathrm{g} / \mathrm{mL})$. Within this jatrophanes, 126 appeared to be the most powerful P-gp inhibitor (Rédei et al. 2012).

Reis et al. (2012) tested MDR reversal potential of jatrophanes pubescene A (270), pubescene C (272), pubescene D (297), euphopubescenol (122), euphopubescene (300), pepluanin D (415), tuckeyanol A (143), and tuckeyanol B (144) and a rearranged polycyclic jatrophane derivative " $1 \beta, 5 \alpha, 14 \alpha, 17 \alpha$-te-

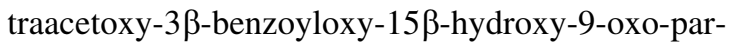
aliane" on COLO320 MDR cells by rhodamine-123 exclusion assay and verapamil was applied as positive control. Both compounds had MDR reversal activity at $2 \mu \mathrm{M}$ and $20 \mu \mathrm{M}$, respectively. Regarding physicochemical properties of compounds, it was showed that the presence of an aromatic moiety in the molecule is important for an increased P-gp affinity. An additional hydrogen bond acceptor connected to the oxygen at C-15 is also important, particularly in the jatrophane scaffold.

Podolski-Renic et al. (2013) evaluated euphodendrophane $\mathrm{H}$ (225) and euphodendrophane S (236) which had been previously isolated from E. dendroides (Jadranin et al. 2013) on cancer cell growth in three human MDR cancer cell lines: NCI-H460/R, colorectal carcinoma DLD1-TxR, and glioma U87TxR by the sulforhodamine B assay (SRB) and their chemo-sensitizing effects in MDR cancer cell lines. 225 and 236 exerted the best inhibitory effect in nonsmall cell lung carcinoma (NSCLC) cell lines: NCI$\mathrm{H} 460$ and NCI-H460/R. However, the $\mathrm{IC}_{50}$ values for 225 differed between sensitive NCI-H460 and resistant NCI-H460/R cells $(6 \mu \mathrm{M}$ and $15 \mu \mathrm{M}$, respectively). Colorectal carcinoma cell lines (DLD1 and DLD1-TxR), as well as the glioma cell lines (U87 and U87-TxR), showed considerably lower sensitivity to the two jatrophanes. These results recapitulated those obtained in the previous study (Aljancic et al. 2011) and indicated the potential of Euph H (225) and Euph S (236) for NSCLC treatment. 225 significantly sensitized NCI-H460/R and DLD1-TxR cells to Paclitaxel (PTX), similar to paclitaxel, $\mathrm{R} \pm$ verapamil (Dex-VER), and tariquidar (TQ); while 236 demonstrated the moderate chemo-sensitizing effect. These observations were in agreement with stronger anti-P- gp activity obtained with 225 in NCI-H460/R and DLD1-TxR. All tested P-gp inhibitors had similar potential for the reversion of PTX resistance. In addition, Dex-VER and TQ showed significantly lower reversal potential in U87-TxR cells as it was expected from single nucleotide polymorphism (SNP) analysis. In conclusion, it was confirmed that jatrophanes stimulate purified tubulin assembly in vitro by this assumption that the mutual effect of PTX and new jatrophanes on microtubule assembly leads to cycle arrest at G2/M phase and partly contributes to Euph H/S and PTX combined effects (Podolski-Renić et al. 2013).

Thirteen new jatrophanes, euphodendrophane G-S (483, and 225-236), and three known compounds (euphodendrophane A (396), euphodendrophane B (397), euphodendrophane F (237)) were isolated from E. dendroides by Jadranin et al. (2013) (Jadranin et al. 2013). The P-gp inhibiting activities of 157-169 had been assessed on previously characterized P-gp overexpressing MDR cancer cell lines: NCI-H460/R, colorectal carcinoma DLD1-TxR, and glioma U87TxR (Pesic et al. 2006; Podolski-Renić et al. 2011). The most promising compounds were euphodendrophane $\mathrm{H}$ and $\mathrm{K}$ (225 and 228), which completely blocked the P-gp pump and demonstrated higher activity than Dex-VER and TQ. However, the effects of 227, 222, and 236 were noteworthy as they had also achieved the complete blockage of P-gp in colorectal MDR cancer cells and exceeded the Dex-VER activity (Jadranin et al. 2013). SAR studies showed no obvious difference in the activity of jatrophanes with 6,17 exo(483, and 225-229, 396, and 397), and those with 5, 6 endo- double bond (234-237). This could be in accordance with the previous findings that said modifications in connectivity made less change in activity than the oxygenation pattern (Corea et al. 2003b). The activity was strongly affected by the OBz group at the positions C-8 and C-9 for jatrophanes with exo- and endo- double bonds, respectively. Wide range of compounds with the same exo- jatrophane skeleton (Corea et al. 2003a) emphasized the importance of free hydroxyl group at $\mathrm{C}-3$ as well as substitution on C-2 and C-5. These conclusions were extended to modified jatrophanes as well (Jadranin et al. 2013).

$\mathrm{Lu}$ et al. (2014) isolated six new jatrophanes (345-349, and 482) from E. sororia. Compounds (345-349, and 482) were evaluated for their capacity 
to inhibit in vitro growth of two human mammary adenocarcinoma (MCF-7) and lung adenocarcinoma (A549) cell lines using a sulforodamine B (SRB) assay. All the compounds were inactive $\left(\mathrm{IC}_{50-}\right.$ $>10 \mu \mathrm{m})$ for the two human cancer cell lines. Compounds (345-349, and 482) were tested for their MDR-reversing activity on KBv200 cells by monitoring the intracellular accumulation of Rho123. Compound 346 was found to be a highly potent inhibitor of efflux pump activity of P-gp in the cancer cells since it was more efficient at $10 \mu \mathrm{M}$ than the standard modulator 'verapamil' (Lu et al. 2014).

Lanzotti et al. (2015) isolated cyparissins A and B (480 and 481) from E. cyparissias and evaluated their ability to inhibit P-gp-mediated MDR and their cytotoxic activity against two human ovarian cancer cell lines, A2780 WT and A2780 ADR. Weak P-gp inhibition was exhibited by 480 and 481 with $\mathrm{IC}_{50}$ of $8.55 \pm 3.21 \mu \mathrm{M}$ and $8.72 \pm 3.45$, respectively comparing to cyclosporine $\mathrm{A}$ (CsA) ( $\mathrm{IC}_{50}$ of $3.37 \pm 1.39 \mu \mathrm{M})$ (Lanzotti et al. 2015). This finding is in agreement with previous SAR studies on jatrophane diterpenes indicating that the presence of both acylation at $\mathrm{C}-3$ and hydroxylation or acylation at C-5 is detrimental for P-gp reversal activity (Corea et al. 2009) and a keto group at C-9 is rather an important feature for cytotoxicity (Vasas et al. 2011). In another study, Barile and Lanzotti isolated presegetanin 16 as well as segetanin A and B (10 and 11), along with four known segetanes (86-89) from $E$. paralias. The cytotoxicity and the anti-MDR activity of all compounds were also tested on human ovarian cancer cells A2780. In a range of concentrations between 0.1 to $10,000 \mathrm{nM}$, none of the tested compounds showed significant activity as compared to controls (Barile and Lanzotti 2007).

Rédei et al. (2015) isolated two new (118 and 269) and one known, isoterracinolide B (23), jatrophanes from E. exigua. P-gp modulatory activities of the compounds on human MDR gene-transfected L5178 mouse lymphoma cells were investigated. In agreement with their earlier published studies, the nature of the substituent at $\mathrm{C}(7)$ influences the ability of jatrophane to enhance intracellular drug accumulation and subsequent MDR reversing activities. It was observed that the activity of 118 and 269 is proportional to the lipophilicity and the size of the ester group at C(7). 269 with two aromatic ester groups was the most lipophilic molecule so it showed maximum activity (FAR $=35.59)$ at $8 \mu \mathrm{g} / \mathrm{mL}$ concentration. 23 displayed similar maximum activity $(\mathrm{FAR}=36.09)$ at $80 \mathrm{mg} / \mathrm{ml}$. Moreover, both compounds had a propanoyl group at $\mathrm{C}(7)$, in contrast to the 7-O-acetyl substituted compound 118, which had the lowest activity (FAR $=25.97)$ (Rédei et al. 2015).

Reis et al. (2015) isolated a rare class of 12,17cyclojatrophanes, (euphowelwitschine A (1), euphowelwitschine B (2), welwitschene (267), epoxywelwitschene (268)) from E. welwitschii. Potential selective antiproliferative activity of the compounds was evaluated against parental gastric (EPG85-257) and pancreatic (EPP-181) human cancer cells. Their drug-selected counterparts resistant to novantrone (RN) and to daunorubicin (RDB), was also evaluated using the SRB assay (Reis et al. 2014). MDR-selective activity was calculated through the relative resistance ratio $\left(\mathrm{RR}=\mathrm{IC}_{50}\right.$ (resistant) $/ \mathrm{IC}_{50}$ (parental) $) . \mathrm{RR}<1$ indicates that the compound kills MDR cells more effectively than parental cells, but if $R R<0.5$, then a collateral sensitivity effect would be taking place. Anti-proliferative selectivity against the resistant gastric cell line EPG85-257RDB was shown by welwitschene (267) $\left(\mathrm{IC}_{50}=17.2 \pm 1.6 \mu \mathrm{M}, \mathrm{RR}=\right.$ $0.6)$ and epoxywelwitschene (268) ( $\mathrm{IC}_{50}$ $=3.6 \pm 0.3 \mu \mathrm{M}, \mathrm{RR}=0.1$ ), with the latter showing a collateral sensitivity effect. For the pancreatic cell lines, an MDR-selective anti-proliferative effect was observed only for 268 against EPP85-181RN ( IC $_{50-}$ $=21.3 \pm 2.5 \mu \mathrm{M}, \mathrm{RR}=0.7)$ and against EPP85$181 \mathrm{RDB}\left(\mathrm{IC}_{50}=18.2 \pm 3.1 \mu \mathrm{M}, \mathrm{RR}=0.6\right)$. It was concluded that epoxywelwitschene (268) can be regarded as a potential MDR reverser (Reis et al. 2015).

Zhu et al. (2016) isolated 13 jatrophanes (238, 192, 49, 193, 206, 194-196, and 186-190) from Pedilanthus tithymaloides eight of which $(238,192,49,193$, 206, and 194-196) were new. Among them, 186-190 had enough yield to design derivatives with different substituents and functions to investigate SAR related to the MDR. Zhu and his coworkers prepared a total of 22 new derivatives through esterification, hydrolysis, or epoxidation modifications. The library containing 35 compounds representing two groups of jatrophanes (I and II) with the presence of 8-OAc or 8-methylene to screen for P-gp dependent MDR modulators. A flow cytometry-based Rho123 effluxion assay was done; the high expressions of P-gp in adriamycin resistant human hepatocellular carcinoma cell line HepG2 
(HepG2/ADR) and adriamycin resistant human breast adenocarcinoma cell line MCF-7 (MCF-7/ADR) were first validated by Western blot. '(1S,2R,3S,4S,7R,9R,13R,14R,15R)-9,15-Diacetoxy1-tosyl-3,7-dibenzyloxy-13,14-dihydroxy jatropha5E,11E-diene' obtained from reaction with tosyl chloride and '(1S,2R,3S,4S,7R,9R,13R,14R,15R)1,9,15-triacetoxy-3,7-dibenzoyloxy-13,14-dihydroxyjatropha-5E,11E-diene' prepared by acetylation and '(1S,2S,3S,4S,7R,9R,13R,14R,15S)-9,15-fiacetoxy-

3,7-dibenzoyloxy-1,13,14-trihydroxyjatropha-5Eene' obtained by treating the solution of 222 with $10 \%$ $\mathrm{Pd} / \mathrm{C}$ under $\mathrm{H}_{2}$ were all identified as potent MDR modulators with greater chemoreversal ability and less cytotoxicity than the third-generation drug 'tariquidar' (TQ). SAR studies showed that increasing the lipophilicity of this class of P-gp inhibitors is beneficial to MDR reversal activity; saturated ring A was essential, while the presence of free hydroxyls on C1C15-C14-C13 fragment had little influence on the activity. In addition, the formation of a rare C5-OC13 bridge would increase the activity, while epoxidation of $\Delta^{12}$ is detrimental to the activity (Zhu et al. 2016).

Reis et al. (2016) screened jatrophanes euphowelwitschine A (1), euphowelwitschine B (2), welwitschene (267), epoxywelwitschene (268) and esulatin M (134) for MDR resistance activity through a combination of Rho123 efflux and chemoreversal assays on adriamycin resistant human hepatocellular carcinoma cell line HepG2 (HepG2/ADR) and adriamycin resistant human mammary adenocarcinoma cell line MCF7 (MCF-7/ADR). 1, 267, 268, and 134 showed to be able to revert the MDR phenotype, at $20 \mu \mathrm{M}$, being two-fold (1 and 134) and three-fold (267 and 268) more effective than 'verapamil' $(\mathrm{FAR}=12.5$ at $20 \mu \mathrm{M}$ ) (Reis et al. 2016). In assays on EPG85257RNOV cells which have been done previously, 268 caused a 4.5 -fold increase of total apoptosis and 134 showed a 2.6-fold increase. Furthermore, both showed a similar effect causing apoptosis in about 2.5 fold for EPG85-257RDB cells (Reis et al. 2015). The compounds 268 and 134 appear particularly interesting, due to their dual activity: as ABCB1 modulators and MDR-selective anti-proliferative compounds. SAR results that high conformational flexibility of the twelve-membered ring of jatrophanes 267, 268, and 134 favored $\mathrm{ABCB} 1$ modulation, in contrast to the
5/8/8 fused ring system of euphowelwitschines A (1) and B (2) (Reis et al. 2016).

Mai et al. (2017a, b) isolated heliosterpenoids A and B (50 and 51) with a novel 5/6/4/6-fused tetracyclic ring skeleton, from E. helioscopia. Their potency for P-gp (ABCB1) inhibitory was evaluated using an adriamycin (ADM)-resistant human breast adenocarcinoma cell line (MCF-7/ADR) (Barile et al. 2008a). Similar inhibitory activity was seen for both compared to $\mathrm{CsA}\left(\mathrm{IC}_{50}=0.49 \mu \mathrm{M}\right)$ with $\mathrm{IC}_{50}$ values of $1.28 \mu \mathrm{M}$ and $1.02 \mu \mathrm{M}$, respectively. Moreover, the cytotoxicity of 50 and 51 was also tested against five human cancer lines (MDA-MB-231, A549, Hela, U118MFG and RKO) by MTT assay. Adriamycin was used as a positive control $\left(\mathrm{IC}_{50}=0.31 \mu \mathrm{M}\right)(\mathrm{Lu}$ et al. 2008). 50 displayed moderate cytotoxicity against MDA-MB-231 cell lines with $\mathrm{IC}_{50}$ value of $24.7 \mu \mathrm{M}$. Both 50 and 51 demonstrated to be new structural potent inhibitors of P-gp (ABCB1) (Mai et al. 2017a).

$\mathrm{Hu}$ et al. (2018) isolated five new $(149,150,322$, 27, and 24) and ten known (22, 345-349, 482, 413, 441 , and 140) jatrophanes from E. sororia. The cytotoxicity and anti-MDR activity of all these compounds were evaluated in a parental DOX-sensitive MCF-7 cell line and its DOX-selected derivative P-gp overexpressing MCF-7/ADR cells by the MTT method. 149 displayed significant MDR reversal activity $\left(\mathrm{IC}_{50}=2.65 \pm 0.33 \mu \mathrm{M}\right)$ in comparison to the other compounds with a low $\mathrm{EC}_{50}$ value $(92.68 \pm 18.28 \mathrm{nM})$ in the MCF-7/ADR cell lines overexpressing P-gp. The remarkable advantages of 149 are its high survival potency toward normal cell line HEK293 $\left(\mathrm{IC}_{50}=98.20 \pm 1.59 \mu \mathrm{M}\right)$ as well as its high therapeutic index (ratio of $\mathrm{IC}_{50}$ toward HEK293 to $\mathrm{EC}_{50}$ for reversing DOX resistance $=1059.56$ ). The results of the Western blot analysis demonstrated that the MDR reversal activity induced by 149 was not due to the inhibition of P-gp expression. The Dixon plot analysis was used to elucidate the type of inhibition. The competitive relationship between the inhibitor and substrate, gave rise to passage of the linear regression line through the origin as it was previously reported (Iseki et al. 1999; Wang et al. 2000). The regression lines of 149 and 'verapamil' coincide with the origin indicating that both were competitive inhibitors of P-gp-mediated DOX transport, which was in accordance with the Lineweaver-Burk analysis. Besides, kinetic characterization revealed that 149 
$(\mathrm{Ki}=0.49-0.50 \mu \mathrm{M})$ possessed a high binding affinity to the DOX recognition site of P-gp with about 5.84- to 5.88-fold lower $\mathrm{Ki}$ values than the average $\mathrm{Ki}$ of 'verapamil'. In this regard, 149 was proven to significantly inhibit DOX transport, increase intracellular DOX concentration, and finally resensitize MCF7/ADR to DOX. They further found that fourfold more of 'verapamil', compared with 149 , is needed to completely restore the DOX accumulation in the MCF-7/ADR cells to the level of the parental MCF-7 cells. Based on SAR study, the activity order of the ester groups at C-5 is 2-methylbutanoyloxy $>$ benzoyloxy $>$ propionyloxy $>$ isobutanoyloxy. Moreover, the presence of an aromatic ester group (benzoyl) at C-14 might increase the modulation potency in comparison to a substituent group of carbonyl or acetoxyl (Hu et al. 2018).

Fang et al. (2018) isolated ES2 (346) from $E$. sororia. They focused on in vitro and in vivo investigation of MDR reversal activity of 346 , as well as elucidation of its underlying mechanisms. The antiproliferative activity of 346 on ABCB1-overexpressing cells (KBv200, MCF-7/ADR, and A549/T) and their parental cells (KB, MCF-7 and A549) were very weak at up to $30 \mathrm{M}$; therefore, the study was performed at a maximum concentration of $10 \mathrm{M}$. 346 considerably increased the sensitivity of KBv200 and MCF-7/ADR), but not their parental cells, to chemotherapeutic drugs (NVB, PTX, and DOX) which are substrates of $\mathrm{ABCB} 1$ at concentration as low as $0.3 \mathrm{M}$; moreover, the reversal effect of 346 was more potent than 'verapamil' at $10 \mathrm{M}$ in both cell lines. These results indicated that 346 can increase the sensitivity of ABCB1- mediated MDR cells to chemotherapeutic agents. The reversal ability of 346 was mainly due to the inhibition of the efflux function of ABCB1 transporter; thus, it increased the intracellular accumulation of chemotherapeutic agents displaying anti-proliferative effects on drug-resistant cells. The drug-efflux function of ABCB1 utilizes energy from ATP hydrolysis, so the rate of ATP hydrolysis is directly proportional to the transport activity of ABCB1. 346 stimulated ABCB1 ATPase activity in a concentration-dependent manner although it had no inhibitory impact on verapamilstimulated ABCB1 ATPase activity; therefore 346 might have direct interaction with ABCB1, which may be different from 'verapamil'. Besides, 346 had no effect on downregulating the protein level of ABCB1.
These results and docking analysis together confirmed 346 induced ABCB1 malfunction may be caused by directly binding to it. These findings suggest the application of 346 in combination with chemotherapeutic agents for cancer treatment (Fang et al. 2018).

Krstic et al. (2018) isolated seven new jatrophanes: nicaeenins A-G (414, and 273-278) together with eight known: euphodendrophanes A-C (396-398), F (237), N (231), O (232), Q (234), and S (236) from $E$. nicaeensis. Their P-gp inhibitory potency was evaluated in two MDR cancer cells (NCI-H460/R and DLD1-TxR). The most potent P-gp inhibitors were 277 with FAR $=4.52 \pm 0.02$ and $5.89 \pm 0.04$ along with 278 with FAR $=5.02 \pm 0.02$ and $4.39 \pm 0.03$ in two mentioned MDR cancer cells lines. 278 also chemosensitized NCI-H460/R cells to DOX stronger than Dex-verapamil due to prolonged effect of P-gp inhibition that remained for seventy-two hours while the effectiveness of 277 was similar to Dex- verapamil. This indicated that the maintenance of the activity against $\mathrm{P}$-gp for a longer period is contributed to the increased reversal potential of jatrophanes. Previous SAR study had shown that two groups of jatrophanes with exo-methylene 6,17 double bond (Jadranin et al. 2013) that lack oxygenation at C-2 and with identical structures except for the substitution at C-8, had a favorable effect on P-gp inhibition (Corea et al. 2003a) upon substitution of $\mathrm{OBz}$ at $\mathrm{C}-8$ with $\mathrm{ONic}, \mathrm{OiBu}$ or OAc. Therefore, it can be demonstrated that 277 and 278 possessing OAc and ONic at C-8 respectively, have moderate but the best potential for P-gp inhibition among tested jatrophanes from $E$. nicaeensis (Krstić et al. 2018).

Mai et al. (2018) isolated two jatrophanes heliojatrones A and B (329 and 7) with a unique trans bicycle (8.3.0) tridecane core, from EtOH extract of the whole plant of E. helioscopia. The inhibitory effect of P-gp mediated ADM efflux by these compounds was evaluated in MCF-7/ADM Cells and cyclosporine A (CsA) was used as positive control (Zhao et al. 2015). Compound $7 \quad\left(\mathrm{IC}_{50}=0.58 \pm 0.05 \mu \mathrm{M}\right)$ showed a remarkable P-gp inhibitory activity in a concentration-dependent manner similar to P-gp inhibitory activity of CsA $(0.84 \pm 0.03 \mu \mathrm{M})$ while weak P-gp inhibitory activities were observed for 329 $(12.03 \pm 4.14 \mu \mathrm{M})$. Therefore compound 7 can be considered as a new structural template for the development of potential MDR reversal agents (Mai et al. 2018a). 
Li et al. (2018) isolated euphornin (208) from $E$. helioscopia in a large amount. Alkaline hydrolysis of 208 using potassium carbonate afforded the main product monodeacetyleuphornin whose structural modification at 14-OH with acyl chlorides yielded 7 alkyl acylated derivatives euphornoate A-G and 14 aryl acylated derivatives euphornoate $\mathrm{H}-\mathrm{U}$ led to a mini library of 21 acylated jatrophane derivatives for expanded SAR studies of MDR modulators ( $\mathrm{Li}$ et al. 2018b). All compounds were tested for their MDR reversal activities in K562/ADR cell using the MTT method to ensure these compounds were non-cytotoxic at tested concentrations $(2 \mu \mathrm{M}$ and $20 \mu \mathrm{M})$. It was seen that the inhibition ratios of all compounds were less than $50 \%$ at $2 \mu \mathrm{M}$ and only two compounds (euphornoate B and euphornoate C) displayed cytotoxic (inhibition ratios were more than 50\%) at $20 \mu \mathrm{M}$; Thus, euphornoate $\mathrm{B}$ and euphornoate $\mathrm{C}$ were not examined for their MDR reversal activity at $20 \mu \mathrm{M}$. The reversal fold values $\left(\mathrm{RF}=\right.$ ratio of $\mathrm{IC}_{50}$ of adriamycin (ADR) alone to $\mathrm{IC}_{50}$ of $\mathrm{ADR}$ in presence of 2 or $20 \mu \mathrm{M}$ sample) were used in evaluating the MDR reversal activity and Verapamil as a positive control. All compounds displayed favorable activities with the RF values over tenfold; over half of them (euphornoate $\mathrm{E}$ and euphornoate $\mathrm{J}$, euphornoate $\mathrm{K}$ and euphornoates M-U) showed reversal ability greater than verapamil. The reversal activities significantly increased at $20 \mu \mathrm{M}$. The RF values of 11 compounds (euphornoates D-F, euphornoate H, euphornoates M$\mathrm{O}$, euphornoate $\mathrm{Q}$, euphornoate $\mathrm{R}$, euphornoate $\mathrm{T}$, euphornoate $\mathrm{U}$ ) were over 100 fold even over 400 fold as the most active (euphornoate $\mathrm{U}$ ) one, and reversal activities of all compounds were greater than the positive control. Previous SAR studies confirmed that substitutions of the "southwestern" fragment (C-2, $\mathrm{C}-3$, and C-5) of jatrophanes, as well as the presence of free hydroxyl at C-15 are important for the activity (Corea et al. 2003a, b). Recently, Zhu et al. (2016) have established the significance of acylation of the free hydroxyl at C-14 as it increases the activity. Therefore, it was aimed to explore different substitutions for expanded SAR studies relative to C-14. Based upon these investigations, the introduction of acyl groups bearing 4 carbons showed the most potent activities at $20 \mu \mathrm{M}$ in alkyl acylated derivatives, for example, euphornoate D (with crotonoyl group) and euphornoate E (with isobutyryl group) exhibited RF values of 393 and 141 respectively. Overall, the MDR reversal activities were better for the aryl acylated derivatives, than the alkyl acylated derivatives at $2 \mu \mathrm{M}$. However, attaching electron withdrawing groups on the aromatic ring, such as nitryl and trifluoromethyl, decreased the activity (euphornoate $\mathrm{K}$ and euphornoate $\mathrm{L}$ ), while electron donating groups, such as methyl and methoxyl, increased the activity (euphornoates M-Q), and the activity of ortho-substituting compound (euphornoate $\mathrm{N}$ ) was higher than meta- and para-substituting compounds (euphornoate $\mathrm{M}$ and euphornoate $\mathrm{O}$ ). Moreover, introducing of an aromatic heterocyclic ring considerably enhanced the activity, as compounds euphornoate T (2-thiophenecarbonyl group) and euphornoate U (2-furoyl group) displayed RF values of 324 and 424 fold respectively. The current SAR studies demonstrated that introduction of an alkyl acyl group bearing 4 carbons at C-14 or an aryl acyl group with electrondonating groups is desirable for the activity and several compounds with RF values over 300 fold at $20 \mu \mathrm{M}$ (euphornoate $\mathrm{D}$, euphornoate $\mathrm{N}$, euphornoate $\mathrm{R}$, euphornoate $\mathrm{T}$, euphornoate $\mathrm{U}$ ) were thought to be promising MDR modulators. This is in agreement with previous SAR studies confirmed the importance of acylation of free hydroxyl at C-14 as it increases the activity (Zhu et al. 2016).

Mai et al. (2018a, b) isolated 17 new jatrophanes, helioscopianoids A-Q (172, 173, 177-180, 6, 220-224, 505, 174-176, and 328), together with eight known, euphornin L (161), euphornin (208), euphornin D (212), euphoscopin F (332), euphoscopin E (331), euphoscopin C (245), euphoscopin B (244), and euphoheliosnoid D (114) from E. helioscopia. P-gp inhibitory effects of helioscopianoids A-Q were evaluated in an adriamycin (ADM)-resistant human breast adenocarcinoma cell line (MCF-7/ADR) where cyclosporin A (CsA) was used as the positive control. Neuroprotective effects were also investigated against serum deprivation-induced and rotenone-induced PC12 cell damage. 220 and 176 enhanced the accumulation of ADM in MCF-7/ADR cells by relatively threefold at a concentration of $20 \mu \mathrm{M}$. Besides, 220 could reduce rotenone-induced $\mathrm{PC} 12$ cell damage, and 173, 220, and 224 displayed neuroprotective activities against serum deprivation-induced PC12 cell damage. SAR studies and corresponding P-gp inhibitory effects demonstrated that the presence of different acyl groups or carbonyl groups at C-7 in the same jatrophane core, especially the butanoyl group instead 
of the hydroxy group at C-7, plays a significant role in their activity (Mai et al. 2018b).

Rédei et al. (2018) isolated novel segetane diterpenoid 77 , new jatrophane compound 279 , along with two other known compounds, segetane 88 and jatrophane 448 from E. taurinensis. The evaluation of the cytotoxic and MDR-reversing activities of them was conducted using flow cytometry measuring the retention of R123 by ABCB1 (P-gp) in MDR mouse $\mathrm{T}$-lymphoma cells overexpressing the $\mathrm{ABCB} 1$ protein and MTT assay. None of the compounds displayed cytotoxic activity on the sensitive parent and resistant MDR cells while all inhibited the ABCB1 MDR efflux pump of the resistant mouse T-lymphoma cells in comparison to 'verapamil', suggesting that they could be used as potential resistance modifiers. Segetane 70 indicated the most potent ABCB1-modulating effect at $20 \mu \mathrm{M}(\mathrm{FAR}=44.44)$ as the first report of the biological activity of segetane-type diterpene (Rédei et al. 2018).

Hasan et al. (2019) isolated 17 new (116, 117, 266, and 307-320) and five known jatrophane diterpenoids (131, 376, 375, 467, and 124) from E. glomerulans. The MDR-reversing activity and cytotoxicity of the new jatrophanes were assessed in the MCF-7 cells and P-gp overexpressing MCF-7/ADR cells using the MTT method. The results demonstrated that these compounds displayed different chemoreversal activities and significantly decreased cytotoxicity. Especially, 314 with $\mathrm{IC}_{50}$ value of $5.0 \pm 0.8 \mu \mathrm{M}$ and 315 with $\mathrm{IC}_{50}$ value of $5.2 \pm 2.0 \mu \mathrm{M}$ afforded MDR reversal activities with RF (reversal fold) values of 12.9 and 12.3 at $10 \mu \mathrm{M}$, respectively, which was as superior as that of 'verapamil' $\left(\mathrm{RF}=13.7, \mathrm{IC}_{50}\right.$ value of $4.7 \pm 0.6 \mu \mathrm{M})$. Because the structurally homogeneous skeletons of (307-309) and (310-320) are different only in the substitution pattern, performing SAR studies was possible. The presence of an isobutanoyloxy moiety at C-8 rather than at C-7 had a positive effect on the modulation of drug accumulation in the MCF-7/ADR cells by comparing the substituents and RF values of 314,315 , and 316 . Furthermore, with the comparison of the biological results of 307,309 , and 311 to those of 308,310 , and 318 , it was demonstrated that favorable following trend in activity at $\mathrm{C}-8$ is: benzoyloxy group $>\mathrm{H} \approx$ hydroxy group (Hasan et al. 2019).
Antiviral activity

Remy and Litaudon (2019) have published a review on anti-CHIKV activity of eighty diterpenoids covering the years 2011 to 2019. Twenty-five jatrophanes have been investigated for their ability to inhibit viral replication. Existence of an acetyl group at position 2 within the 9,14-dioxojatropha-dienes and the 2-methylbutyryl group in the 9-oxojatropha-dienes series, proved to be deleterious for anti-CHIKV activity. Furthermore, the C-8 substitution influences the activity of jatrophanes (tiglyloxy $>$ benzoyloxy $>$ acetyloxy $\approx$ isobutyryloxy) (Remy and Litaudon 2019).

Esposito et al. (2016) isolated six new jatrophane esters including euphodendroidins J-O (456-461) from the $E$. dendroides. The assessment of the antiviral activity of these compounds was performed in a virus-cell-based assay for the Chikungunya (CHIK) virus. 460 and 461 showed moderate antimetabolic effects on Vero cells (Esposito et al. 2016).

Nothias-Scaglia et al. (2014) evaluated the antiCHIKV capacity of six new $(367,368$, and 500-503) together with six known (488, 493, 484, and 359-361) jatrophanes from E. amygdaloides. The compounds were classified into two groups A and B. Group A (500-503, 488, 359, and 493) were the esters of ' 9,14 dioxojatropha-6(17),11E-diene' and group B (367, 368 , and $484,360,361$ ) were those of '9-oxojatropha$6(17), 11 \mathrm{E}$-diene'. The selective antiviral activity was investigated against CHIK virus with two additional members of the genus alphavirus (Sindbis virus (SINV) and Semliki Forest virus (SFV)) and two members of the genus Lentivirus, i.e. human immunodeficiency virus (HIV)-1 and HIV-2 viruses. Regarding the antiviral activity against alphaviruses, 502 and to a lesser extent 503 were found to be potent selective inhibitors of CHIKV replication (half $=$ maximal effective concentration $\left(\mathrm{EC}_{50}\right)=0.76 \pm 0.14 \mu \mathrm{M}$, selectivity index (SI) $=208$, and $\mathrm{EC}_{50}$ $=4.3 \pm 0.2 \mu \mathrm{M}, \mathrm{SI}=29$, respectively). 502 also exhibited moderate anti-SINV activity, while 360 , 367, and 368 showed a significant, albeit weak, antiviral activity on the replication of SINV and SFV. Concerning the activity of group A compounds, since 359 and 493 were weakly active, it could be concluded that the presence of an acetyl group at position 2 might be detrimental for anti-CHIKV activity (cf. 502 vs. 359, and 503 vs. 493). The 
influence of the C-8 substitution as shown by the comparison of anti-CHIKV activities of (500-502 and $488,359,493)$ was the other substantial chemical feature. In this regard, the following sequence inefficiency at C-8 was proposed: tiglyloxy $>$ benzoyloxy $>$ acetyloxy $\approx$ isobutyryloxy. Only 360 and 367 of group $\mathrm{B}$ showed significant anti-CHIKV activities $\left(\mathrm{EC}_{50}=19.5 \pm 3.6 \mu \mathrm{M}, \mathrm{SI}=7.8 ; \quad \mathrm{EC}_{50-}\right.$ $=21.0 \pm 3.4 \mu \mathrm{M}, \quad \mathrm{SI}=2.8$, respectively). These results proved that the acetyl group plays an equivalent role as an isobutyryl substituent at C-8 in the antiCHIKV activity. Contrarily, since 368 was much less active, it was deduced that the 2-methylbutyryl group might be detrimental for this activity. In virus-cellbased assay for HIV, only 502 showed a strong selective antiviral effect on HIV-1 and HIV-2 virus replication, with $\mathrm{IC}_{50}=0.34 \pm 0.05 \mu \mathrm{M}$, SI $>96$ and $\mathrm{IC}_{50}=0.043 \pm 0.005 \mu \mathrm{M}, \quad \mathrm{SI}>751$, respectively. 503, 488, 359, and 360 displayed moderate antiviral activity against HIV-2 (Nothias-Scaglia et al. 2014).

Bedoya et al. (2009) investigated the anti-HIV activity of SJ-23b (488) previously isolated from $E$. hyberna (Appendino et al. 2002) to identify the potent natural or synthetic PKC agonists lacking tumor promoter and cellular proliferative activities for treatment of HIV-1 latency in combination with HAART (highly active antiretroviral therapy). Although 'prostratin' a non-tumorogenic phorbol ester, is a favorable lead compound to antagonize HIV-1 latency, the high concentrations required may prevent its clinical use. SJ-23b with at least one order of magnitude more potent than 'prostratin' internalized the HIV-1 receptors (CD4, CXCR4, and CCR5) and prevented de novo viral infection in human primary $\mathrm{T}$ cells at the nanomolar range. Due to their mechanisms of action, short cycles of treatment with these small molecules inducing HIV reactivation combined with HAART, could contribute to a decrease in viral reservoirs. Concerning this investigation, SJ-23b can be considered as an adjuvant therapy agent to target latent reservoirs for patients on HAART (Bedoya et al. 2009).

Abdelgaleil et al. (2001) isolated two new segetanes named segetanes A (80) and B (81) along with five jatrophanes (484-487 and 489) and four segetanes (84, 85, 88, and 89) and two paralianes (72 and 73), which had previously been isolated from the same plant collected in Turkey (Öksüz et al. 1997) and
Spain (Jakupovic et al. 1998c), and from Euphorbia segetalis (Jakupovic et al. 1998a). Antiviral activity against HIV-1 replication was tested on the inhibition of virus-induced cytopathicity in MT-4 cells. 88 and 485,487 , and 489 showed weak activities in a range of $\mathrm{EC}_{50}=40-79 \mu \mathrm{g} / \mathrm{mL}$ and compound 72 showed a moderate antiviral activity $(\mathrm{EC} 50=14 \mathrm{mg} / \mathrm{ml})(\mathrm{Ab}-$ delgaleil et al. 2001).

Mai et al. (2017a, b) isolated secoheliosphanes A (8) and B (9) and secoheliospholane A (17) together with 2-epieuphornin I (219) and euphoscopin A (242) from E. helioscopia. Antiviral activity of all isolated compounds was evaluated against the herpes simplex virus 1 (HSV-1) using Vero cells (Lv et al. 2016). 'Acyclovir' (ACV) was used as the positive control, with $\mathrm{IC}_{50}$ values of $0.41 \mu \mathrm{M}$. Among all, 9 displayed moderate activity against $\mathrm{HSV}-1$ with $\mathrm{IC}_{50}$ value of $6.41 \mu \mathrm{M}$. The bioassay data demonstrated that secojatrophane skeleton $(8,9$, and 17) has stronger antiviral activity against HSV-1 than its precursor (242) possessing jatrophane skeleton (Mai et al. 2017b).

Esposito et al. (2016) isolated six new jatrophanes: euphodendroidins J (456), K (457), L (458), M (459), $\mathrm{N}$ (460), and $\mathrm{O}$ (461) together with nine known diterpenoids: euphodendroidins A (417), B (418), E (421), and F (422), 13 $\alpha$-hydroxyterracinolides $G$ and B (28 and 47), and terracinolides J and C (32 and 38) from E. dendroides. The antiviral activity of all compounds was conducted in a virus-cell-based assay for the CHIK virus. 32 displayed anti-CHIKV activity with $\mathrm{EC}_{50}$ values of $15.0 \pm 3.8 \mu \mathrm{M}$ and showed a remarkable anti-metabolic effect only at concentrations of $36 \pm 3.1 \mu \mathrm{M}$, allowing the calculation of selectivity index (SI) of 2.4 (Esposito et al. 2017).

Antifungal activity

Rawal et al. (2014) isolated euphosquamosins A-C (184, 185, and 350) along with guyonianin B (148) and Deacetylserrulatin B and euphoscopin C (245) from $E$. squamosa and evaluated their ability to inhibit drug efflux by multidrug transporters of Candida albicans. Deacetylserrulatin B and euphosquamosin C (350) strongly inhibited the drug-efflux activity of the primary $\mathrm{ABC}$-transporter $\mathrm{CaCdr} 1 \mathrm{p}$, an effect that was translated into increased sensitivity to fluconazole. These compounds were transported by CaCdr1p, as shown by observation of an 11- to 14-fold cross- 
resistance of yeast growth, and could also inhibit the secondary major facilitator superfamily (MFS)-transporter CaMdr1p. In contrast, euphosquamosin A (184) was selective for $\mathrm{CaCdr} 1 \mathrm{p}$, possibly as a result of a different binding mode. Taken together, these observations suggested the jatrophane diterpenes be a new class of potent inhibitors of multidrug transporters critical for drug resistance in pathogenic yeasts (Rawal et al. 2014).

Nim et al. (2016) investigated the jatrophanes euphopubescenol (122), euphomelliferene A (294), euphomelliferene B (295), and euphomelliferine (293) for their inhibitory effect on drug efflux activity of Candida albicans CaCdr1p and CaMdr1p multidrug transporters overexpressed in a Saccharomyces cerevisiae strain. Their inhibitory potency was evaluated through a functional assay of Nile Red accumulation monitored by flow cytometry. A chemosensitization assay, using the checkerboard method, was also utilized to evaluate their type of interaction with fluconazole. In the transport assay, most compounds were found to suppress both transporters as shown by relative resistance indices close to unity. In contrast, 122 and 294 were selective for CaMdr1p and CaCdrlp, respectively. Moreover, when used in combination with fluconazole, 295 and 293 exhibited strong synergistic interactions (fractional inhibitory concentration index $(\mathrm{FICi})=0.071$ ) against the yeast strain overexpressing CaMdr1p by a 13 -fold decrement of the minimum inhibitory concentration for $80 \%\left(\mathrm{MIC}_{80}\right)$ of the antifungal agent. Both compounds were also able to reduce the effective concentration of this antifungal agent by 4- to eightfold against an azole-resistant clinical isolate of Candida albicans (Nim et al. 2016).

Esposito et al. (2017) isolated twenty-nine jatrophanes from E. semiperfoliata (501, 502, 367, 488, 493, 484, 360, 368, 361, and 359) and $E$. dendroides $(28,47,32,38,421,422,417,457-461,451-455$, and 462) five of which (451-455), were new (Esposito et al. 2017). The ability of these compounds to modulate drug efflux by multidrug transporters of Candida albicans was assessed in Saccharomyces cerevisiae strain overexpressing either $\mathrm{CaCdr} 1 \mathrm{p}$ or CaMdr1p. Cytotoxicity of active compounds on a $C$. albicans MDR strain evaluated in the second bioassay along with their ability to sensitize yeast growth through synergistic interaction with fluconazole. 462 was selective for CaCdrlp and induced a strong Nile
Red (NR) accumulation (92\%) through inhibition of CaCdr1p mediated efflux, whereas 367 was selective for CaMdr1p, with a $74 \%$ NR accumulation. In contrast, 28 and 418 were found to accumulate the Nile Red (NR) mediated by the two multidrug transporters, at 8--64\% for CaCdr1p and $79-65 \%$ for CaMdr1p. The ability of the 367, 28, 462, and 418 showed potent inhibition of the MDR transporters Cdr1p and Mdr1p to sensitize yeast growth through the antifungal agent fluconazole was evaluated by the checkerboard method (White et al. 1996). Regarding these results, 367, 28, 462, and 418 displayed high fractional inhibitory index (FICI) values $(\geq 1 \mu \mathrm{M})$, revealing that, despite their ability to inhibit $C$. albicans MDR transporters expressed in yeast strains, they were not able to induce sensitization to fluconazole for C. albicans-resistant strain growth. However, some jatrophanes were found to be selective or dual inhibitors against the yeast MDR transporters $\mathrm{CaC}$ dr1p and CaMdr1p, but only deacetylserrulatin B and 350 obtained from E. squamosa and 295 and 293 isolated from $E$. mellifera were able to sensitize the $C$. albicans MDR strain to fluconazole (Nim et al. 2016; Rawal et al. 2014). Even with structural similarity of mentioned jatrophanes with the latter substance, none of them exhibited a similar biological activity; therefore, the ability of inhibitors to sensitize yeast growth to the antifungal activity of fluconazole is mainly dependent on the nature, number, and position of functional groups on the macrocyclic core. To apply SAR study, the chemical space of diterpenoids, was classified into A to D groups (group A: esters of '9,14dioxojatropha-6(17),11E-diene' (501-503, 488, 359, and 493), group B: esters of '9-oxojatropha6(17),11E-diene' (360, 367, 484, and 360), group C: esters of '14-oxojatropha-6(17),11E-diene' (28, 47, $32,38,421,422,417,457-461,451-455$, and 462), and group D: esters of '17-bishomojatrophane' (28-31)). The potency and selectivity of compounds are sensitive to the substitution pattern on the jatrophane skeleton. Hydrophobicity and an electron acceptor moiety are essential factors for the recognition of diterpenes with P-gp multidrug transporters in human cancer cell lines (Ferreira et al. 2011). But according to the results of $84.9 \%$ of the data set variance (PC1: $50.4 \%, \mathrm{PC} 2: 24.5 \%$, and $\mathrm{PC} 3: 10.0 \%$ ) it was demonstrated that the modulation of CaMdr1p and $\mathrm{CaCdr} 1 \mathrm{p}$ multidrug transporters in $S$. cerevisiae by $462,28,367$, and 418 could not be affected by the 
hydrophobicity or by other chemical properties used in this PCA analysis. It was instead concluded that difference in acylation pattern between 367 with 361 and 484 from group B, appears to play an important role for a strong inhibition of the CaMdr1p multidrug transporter. An isobutyrate group at C-8 in 367, instead of an acetoxy group or hydroxy group in 360 and 484, respectively. Moreover, the comparison of 418 with 417 and 460 from group $C$ showed that the presence of a methylbutyrate group at C-5, instead of either an isobutyrate group or a benzoate group, respectively, had a significant contribution to the inhibition of CaCdr1p activity. Besides, since 29 was much less active than 28 , it was indicated that the acetoxy group at $\mathrm{C}-15$ was detrimental for inhibiting drug-efflux activities of both CaCdr1p and CaMdrlp transporters. Regarding complex conformational behavior of jatrophanes depending on their esterification pattern (Esposito et al. 2016; Günther et al. 1998), it was deduced that the modulation of CaMdr1p and CaCdr1p multidrug transporters by jatrophanes could rely on their conformational characteristics. In conclusion, 30 and 28 from group $C$ and 417, 418, and 421 from group $\mathrm{D}$ were shown to be promising candidates for the development of P-gp modulators to tackle MDR human cancer cell lines. These results also demonstrated that macrocyclic diterpenoids, which are able to reverse the MDR of cancer cell lines overexpressing P-gp transporters, have not necessarily the ability of chemosensitizing $C$. albicans MDR strains (CaCDR1 and CaMDR1), revealing a possibly different binding mode (Esposito et al. 2017).

Anti-inflammatory activity

The Nitric Oxide (NO) produced by iNOS in macrophages is involved in various inflammatory diseases and therefore inhibitors of NO production may have potential therapeutic value as anti-inflammatory agents.

Barile et al. (2008a, b) evaluated in vivo antiinflammatory potential of a set of over sixty structurally-homogeneous diterpenes belonging to the rare classes of pepluane and paraliane. The results showed the importance of functionality and structure of the D-ring for the activity and its possible involvement in the inhibition of NF- $\kappa \mathrm{B}$ activation as follows (Fig. 14) (Barile et al. 2008b):

Lee et al. (2016) isolated two new jatrophanes: kanesulones A (323) and B (324) together with six known jatrophanes: kansuinin A (95), B (477), D (97), E (371), H (96), and esulone A (298) from E. kansui. The inhibition of NO production was tested in lipopolysaccharide (LPS)-stimulated RAW 264.7 cells, with 'aminoguanidine' as the positive control $(\mathrm{IC} 50=18.7 \mu \mathrm{M})$. It was demonstrated that all

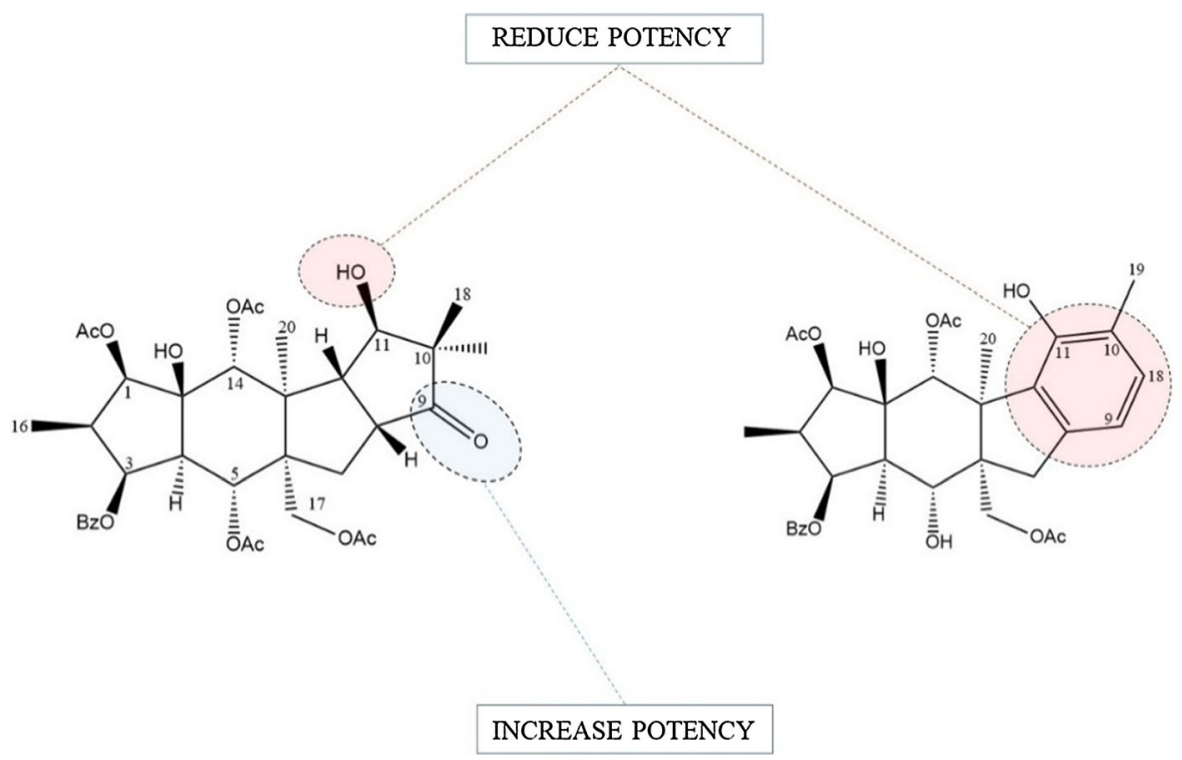

Fig. 14 Key pharmacophoric elements for the anti-inflammatory activity 
compounds exhibited inhibitory effects on LPSinduced NO production which possessed lipophilic substituents such as benzoyl, nicotinoyl and decadienoyl moieties with $\mathrm{IC}_{50}$ values ranging from 0.7 to $46.5 \mu \mathrm{M}$ in RAW 264.7 macrophages (Lee et al. 2016).

Wan et al. (2016a, b) isolated paralianones A-D (66-69) and pepluanols A-H (52-56, 58, 62, and 63), along with five known compounds 72 (Jakupovic et al. 1998b), 59 (Hohmann et al. 1999a), 60 (Hohmann et al. 2000a), 61 (Hohmann et al. 1999b), and pepluanone (57) (Corea et al. 2005b), from $E$. peplus.The isolated compounds were tested for inhibitory activity on LPS-stimulated NO production in RAW264.7 macrophage cell line. Compounds 68, $69,62,72$, and 61 displayed inhibitory effects on NO inhibition, with $\mathrm{IC}_{50}$ values of $33.7,38.3,36.6,29.9$, and $37.1 \mu \mathrm{M}$, respectively. In addition, none of the test compounds displayed any obvious cytotoxicity to RAW264.7 cells (Wan et al. 2016a).

Chen et al. (2014) isolated new compounds 239-241, and 330 along with five known analogues: $332,247,337,245$, and 256 from E. helioscopia. The inhibitory activity of the compounds was investigated on LPS-induced NO production in murine microglial BV-2 cells. All tested diterpenes exhibited inhibitory effects on LPS-induced NO production. 330 and five aforementioned known analogues inhibited LPS-induced NO production dose-dependently with $\mathrm{IC}_{50}$ values of $41.9,25.5,17.5,45.2,20.9$, and $27.3 \mu \mathrm{M}$, respectively. 239, 241, 337, and 256 showed moderate inhibitory effects while 247 and 245 showed weak activity $\left(\mathrm{IC}_{50}\right.$ values $\left.>100 \mu \mathrm{M}\right)$. All the assayed compounds had no considerable cytotoxicity to the BV-2 cells at their effective concentration for the inhibition of NO production in MTT assay. It could be deduced that these bioactive diterpenes, especially 330 and 332 with strong NO inhibitory activities, might be considered as impressive agents in various inflammatory diseases (Chen et al. 2014).

Barile and Lanzotti (2007) isolated two new diterpenes, paralianone (71) and pepluene (64) together with two known analogues (76 and 65) from Euphorbia paralias. The ability of the isolated compounds as an anti-inflammatory agent on LPS-stimulated NO production in RAW264.7 macrophage cell line. Compound 76 showed the highest anti-inflammatory activity comparable to those recently discovered for pepluanone (57). Comparison of the activity of paralianes (71 and 76) and pepluanes (64 and 65) demonstrated the crucial role of a carbonyl on D-ring and negative effects when D-ring is hydroxylated or aromatic (Barile et al. 2007).

Corea (2005) isolated a new diterpene, pepluanone (57) together with a known pepluane diterpene (61) (Hohmann et al. 1999a; Jakupovic et al. 1998b) from Euphorbia peplus L. The ability of pepluanone (57) as an anti-inflammatory agent on LPS-stimulated NO production in RAW264.7 macrophage cell line. The results showed that pepluanone inhibited in a concentration-dependent manner, without cytotoxicity, both LPS-induced NO and PGE2 productions. It was also able to inhibit TNF-R mRNA expression. The mechanism by which pepluanone inhibits iNOS, COX-2, and TNF-R mRNA involves the suppression of NF- $\kappa \mathrm{B}$ activation. Comparison of high in vivo efficiency of pepluanone and the absence of notable in vivo activity for compound 61 highlighted the importance of functionality at C-9 (a ketone in pepluanone and an acetoxyl in compound 61 (Corea et al. 2005b).

\section{Anti-arrhythmic effect}

In heart muscle, $G$ protein-coupled inwardly rectifying potassium (GIRK) channels are responsible for $\mathrm{K}^{+}$-fluxes and membrane repolarisation and/or hyperpolarisation. These ion channels selectively expressed in the cardiac atrium are not present in the ventricle. Electrical remodeling of atrial heart muscle during chronic atrial fibrillation may result in a constitutively active form of the GIRK channel, which may indeed lead to a better understanding of the important role this channel plays in said disease. Kúsz et al. (2016) investigated electrophysiological effects of guyonianin G (280) and H (281), together with four jatrophanes 326, 302, 372, and 5 previously isolated from E. mongolica (Rédei et al. 2012), E. serrulata (Hohmann et al. 2002), and E. salicifolia (Hohmann et al. 2001a, b) on stable transfected HEK-GIRK1/4 (Kir3.4) cell lines (Kúsz et al. 2016). It was demonstrated that jatrophanes could be new inhibitors with high potency for atrial GIRK channels for the first time. The most significant dose-dependent inhibitory effects on GIRK channel were displayed by guyonianin $\mathrm{G}$ (280) and H (281). Guyonianin G (280) had the highest blocking activity $(70 \%$ at $10 \mu \mathrm{M})$, while the inhibition of GIRK current by guyonianin H (281) at $10 \mu \mathrm{M}$ was $48 \% .484$ also inhibited the GIRK current 
with similar activity to guyonianin $\mathrm{H}(44 \%$ at $10 \mu \mathrm{M})$. Besides, this substance illustrated a quick effect occurred immediately after the application. Low blocking activity was observed in diterpenes 302 and 372 while 5 did not represent any remarkable effect (Kúsz et al. 2016). Selective inhibition of the GIRK channel might lead to develope a promising class of new antiarrhythmic drugs (Dobrev et al. 2005; Hashimoto et al. 2006; Kobayashi and Ikeda 2006).

Kusz et al. (2018) isolated nine new (151-159) and two known (euphomelliferene B (295), euphornin (208)) jatrophanes from E. dulcis. The electrophysiological effects of 151, 152, 159, 295, and 208 were assayed in the HEK-hERG cell line. In order to find effective natural agents for the treatment of atrial fibrillation, the selectivity of their GIRK blocking effect on stable transfected HEKGIRK1/4 (Kir3.1/3.4) and HEK-hERG (Kv11.1) cell lines in two concentrations ( 1 and $10 \mu \mathrm{M})$ was examined. Almost all of the diterpenoids showed remarkable blocking activity on GIRK channels at $10 \mu \mathrm{M}$ concentration $(60.8-88.7 \%)$ and displayed considerable inhibitory effects even at $1 \mu \mathrm{M}$ concentration. None of the tested jatrophanes interfered with the function of hERG proteins. Therefore, jatrophane diterpenoids might be suggested as a group of potential lead compounds for novel therapeutic agents against atrial fibrillation (Kusz et al. 2018).

\section{Antibacterial activity}

Japodagrone (90) which had previously been isolated from Jatropha podagrica was investigated for its antibacterial activities on staphylococcus aureus (ATCC29213), bacillus subtilis (ATCC6051), escherichia coli (ATCC25922), and pseudomonas aeruginosa (ATCC 27853) by Aiyelaagbe et al. (2007). Streptomycin and gentamycin $(20 \mu \mathrm{g} / \mathrm{disk})$ were used as positive controls. It was observed that japodagrone (90) only showed activity against Bacillus subtilis (ATCC6051), giving a zone of $12 \mathrm{~mm}$ at $20 \mu \mathrm{g} / \mathrm{disk}$. This compound was presumed to be responsible for some of the antibacterial activity exhibited by extracts of this plant (Aiyelaagbe et al. 2007).

Neuroprotective effect

Pan et al. (2004) isolated kansuinines F (369), G (370), and $\mathrm{H}$ (96) together with four known jatrophanes: kansuinines D (97), E (371), A (95), and ' $3 \beta, 5 \alpha, 7 \beta, 15 \beta$-tetraacetoxy- $9 \alpha$-nicotinoyloxy jatropha-6(17)-11E-dien-14-one' from E. kansui. The ability of different kansuinins to activate tropomyosin receptor kinase (Trk) A and Trk B signaling was tested by determining the survival effects of these compounds on fibroblasts expressing Trk A and Trk B. The survival of these cells is solely dependent on nerve growth factor (NGF) and BDNF (a member of the neurotrophin family related to NGF) treatment and they would normally die in the absence of NGF and BDNF. NGF had been the best candidate for its neuroprotective effects in the animal models of neurodegenerative diseases. The ability of small molecules like kansuinins which mimic or induce NGF activity was investigated for the treatment of Alzheimer. Kansuinin E (371) showed a particular effect on the survival of TrkA fibroblasts compared with TrkB cells with an $\mathrm{ED}_{50}$ value of $0.23 \mu \mathrm{g} / \mathrm{mL}$. In contrast, kansuinins A (95), D (97), and F (369) enhanced the survival of both Trk A- and Trk B-expressing fibroblasts (Pan et al. 2004).

\section{Antithrombotic activity}

Activated protein kinase $\mathrm{C}$ ( $\mathrm{PKC}$ ) phosphorylates and regulates downstream substrates that can lead to platelet activation, secretion, and aggregation, which are indeed important for thrombus formation. Therefore PKC plays an outstanding role in the occurrence of thrombotic diseases. Several tumorpromoting Euphorbia diterpenes are known to act as direct activators of PKC, but many types of such diterpenes have not been studied as platelet stimulators yet. Tsai et al. (2016) studied esulatins B and I (470 and 128) previously obtained from E. esula for their effects on PKC activation and platelet stimulation. These compounds did not induce platelet aggregation at a concentration as high as $10 \mu \mathrm{M}$. Furthermore, these compounds which were inactive in the platelet aggregation assay failed to induce platelet secretion but the mentioned research suggests that platelets appear to be a useful model for screening PKC activators of natural origin or their chemical derivatives (Tsai et al. 2016). 
Antimalarial activity

Zhou et al. (2016) isolated 14 new diterpenoids, named euphorbesulins A-O (13-15, 282-292, and 70). These euphorbesulins included presegetane (13-15) and jatrophanes (282-292) and one paraliane (70). Tested jatrophanes (13-23) isolated from E. esula for antimalarial effects against chloroquine-resistant plasmodium falciparum strain Dd2 using a SYBR-Green assay with artemisinin as the positive control. Euphorbesulin G (285) exhibited low nanomolar antimalarial activity $\left(\mathrm{IC}_{50}=0.12 \pm 0.04\right)$, while the rest showed only moderate to no antimalarial activity $\left(\mathrm{IC}_{50}>5\right.$ ) (Zhou et al. 2016).

Mongkolvisut and Sutthivaiyakit (2007) isolated six new poly-O-acylated jatrophanes (186-191) from the Pedilanthus tithymaloides. Their biological activities including antimalarial effects against plasmodium falciparum $\mathrm{K} 1$ strain as well as antitubercular effects against mycobacterium tuberculosis H37 Ra were investigated. 186 and 188-190 were found to be active against $P$. falciparum with $\mathrm{IC}_{50}$ values of 3.4-4.4 $\mu \mathrm{g} / \mathrm{mL}$; whereas, 187 was inactive at $10 \mu \mathrm{g} /$ $\mathrm{mL}$. Considering the anti-mycobacterial activity, 186 was the most active while 187-190 have shown moderate to mild activity. An additional anti-fungal assay was applied on 186, 187, and 190 against Candida albicans at $50 \mu \mathrm{g} / \mathrm{mL}$, none of which was active (Mongkolvisut and Sutthivaiyakit 2007).

Lipid-lowering agent

Li et al. (2018a, b) isolated 33 jatrophanes, seven of which $(215-217,321,12,169$, and 170) were new and others were known compounds identified as euphoscopin A-D (242, 244, 245, and 106), euphoheliosnoid A (337), euphoscopin E-F (331 and 332), euphorbiapene D (330), euphoscopin J (250), euphorbiapene A (239), epieuphoscopin A and B (252 and 254), epieuphoscopin D (107), epieuphoscopin F (335), euphornin L (161), euphorbiapene C (241), euphornin (208), euphornin A (210), euphornin C and D (265 and 212), euphornin H and I (259 and 260), euphornin G (258), euphornin J and K (262 and 263), euphorbiapene B (240), euphoheliosnoid B (338) from $E$. helioscopia. This mini-library of jatrophanes was established to screen hit or lead compounds possessing lipid-lowering activity. LDL-uptake screening assay showed that most of them improved LDL-uptake rate in HepG2 cells, with 239, 161, and 212 displaying superior effects. It was further found that these three compounds could enhance LDLR protein level in HepG2 cells dose-dependently. SAR studies demonstrated that the type of substitution at C-9 is essential for the activity, as replacing carbonyl with acetoxy group considerably increased the activity of 259 vs. 212. However, different substitutions at C-7 look to be less effective on the activity, as compounds (215-217), (242, 244, 245, 106, and 337) and (208, 210, and 265) displayed similar results. 239 with a long conjugated fragment from C-5 to the carbonyl exhibited significantly increased activity. Besides, large steric hindrance between C-14 and C-15 is unpleasant for the activity, as 208, 210, and 265 displayed weak activities although they all have an acetoxy group at C-9. The presence of a carbonyl or an acetoxy group at C-14 also did not influence activity ( 242 vs. 331,244 v.s 332,245 vs. 330 ). Furthermore, similar activities were seen for 244 vs. 262,245 vs. 240, 337 vs. 338,252 vs. 260, and 241 vs. 256 demonstrating that configuration at $\mathrm{C}-2$ was not important for the activity, same being true for configuration at C-13 as the results of 242 vs. 252, 106 vs. 107,259 vs. 262 had been shown this matter. Among all, 161 showed a notable lowering effect in serum CHOL and LDL-C levels while it was less effective on HDL-C level and body weight in vivo ( $\mathrm{Li}$ et al. 2018a).

\section{Conclusion}

Jatrophanes isolated from Euphorbia species and their polycyclic rearranged derivatives with a wide chemical structural diversity have been mentioned recently as privileged bio-resources for the development of potential drugs (Jassbi 2006; Kirby et al. 2010). Jatrophanes and modified jatrophanes have exhibited a large number of biological activities, such as being inhibitors of CaCdr1p and/or CaMdr1p efflux pumps in Candida albicans (Esposito et al. 2017), modulators of P-glycoprotein (P-gp) exhibiting reverse multidrug resistance (MDR) (Corea et al. 2003a; Hohmann et al. 2002), paclitaxel-like microtubule interacting activity (Miglietta et al. 2003), moderate cytotoxic agents against a variety of cancer cells (Hegazy et al. 2010; Lanzotti et al. 2015), and promising synergistic agents increasing the anticancer activity on resistant cells in 
combination with anticancer drugs (Pešić et al. 2011). The most studied biological activity of them is the MDR reversing activity in cancer cells overexpressing P-gp. Jatropha-5,12-diene inhibitors of the drug efflux transporters ABCB1 P-gp and ABCG2 have been developed (Reis et al. 2016). SAR studies provide invaluable information on the key pharmacophoric elements of these compounds. Furthermore, they give the possibility to evaluate the efficacy of different ester groups on each position of the jatrophane macrolides requiring no costly, time-consuming labor-intensive synthesis or semisynthetic modifications. These findings coupled with conformational flexibility of twelve-membered jatrophane core have suggested that conformational flexibility is determinant for P-gp modulation, and in MDR activity, they are generally more active than the rearranged polycyclic ones (Ferreira et al. 2014; Reis et al. 2012). Besides, the hydrophobicity along with the substitutions of the "southwestern" fragment (C-2, C-3, and C-5) are also critical factors for the activity of jatrophane-type diterpenes (Corea et al. 2004a). Jatrophanes exhibit also anti-inflammatory activities (Chen et al. 2014; Lee et al. 2016). They have also been reported for in vitro antiviral activity against human immunodeficiency (Bedoya et al. 2009) and Chikungunya viruses (Nothias-Scaglia et al. 2014). It had been proposed that short cycles of treatment with these small molecules inducing HIV reactivation combined with highly active antiretroviral therapy (HAART), could contribute to a decrease of viral reservoirs (Bedoya et al. 2009; Nothias-Scaglia et al. 2014). Jatrophanes like pubescenol and pubescene D showed considerable antiproliferative activity against human tumor cell lines MCF-7, NCI-H460, and SF-268 (Valente et al. 2004a, c). Thus, more clinical trials and structural analysis will be required in the future to evaluate the clinical benefits of these recently discovered compounds.

\section{References}

Abdelgaleil SA, Kassem SM, Doe M, Baba M, Nakatani M (2001) Diterpenoids from Euphorbia paralias. Phytochemistry 58(7):1135-1139

Adolf W, Hecker E (1977) Diterpenoid irritants and cocarcinogens in Euphorbiaceae and Thymelaeaceae: Structural relationships in view of their biogenesis. Isr $\mathrm{J}$ Chem 16(1):75-83

Ahmed AA, Gherraf N, El-Bassuony AA, Rhouati S, Gad MH, Ohta S, Hirata T (2006) Guyonianin A and B, two polyester diterpenes from Algerian Euphorbia guyoniana. Nat Prod Commun 1(4):1934578X0600100402

Aiyelaagbe OO, Adesogan K, Ekundayo O, Gloer JB (2007) Antibacterial diterpenoids from Jatropha podagrica Hook. Phytochemistry 68(19):2420-2425

Aljancic IS, Pešić M, Milosavljevic SM, Todorovic NM, Jadranin $\mathrm{M}$, Milosavljevic G, Povrenovic D, Bankovic J, Tanic N, Markovic ID (2011) Isolation and biological evaluation of jatrophane diterpenoids from Euphorbia dendroides. J Nat Prod 74(7):1613-1620

Amaral L, Spengler G, Molnár J (2016) Identification of important compounds isolated from natural sources that have activity against multidrug-resistant cancer cell lines: effects on proliferation, apoptotic mechanism and the efflux pump responsible for multi-resistance phenotype. Anti-cancer Res 36(11):5665-5672

Appendino G (2016) Ingenane diterpenoids, vol 102. Prog Chem Org Nat Prod. Springer, Berlin, pp 1-90

Appendino G, Szallasi A (1997) Euphorbium: modern research on its active principle, resiniferatoxin, revives an ancient medicine. Life Sci 60(10):681-696

Appendino G, Jakupovic S, Tron GC, Jakupovic J, Milon V, Ballero M (1998) Macrocyclic Diterpenoids from Euphorbia semiperfoliata. J Nat Prod 61(6):749-756

Appendino G, Belloro E, Tron GC, Jakupovic J, Ballero M (2000) Polycyclic diterpenoids from Euphorbia characias. Fitoterapia 71(2):134-142

Appendino G, Spagliardi P, Ballero M, Seu G (2002) Macrocyclic diterpenoids from Euphorbia hyberna L. subsp. insularis and their reaction with oxyphilic reagents. Fitoterapia 73(7-8):576-582

Ayatollahi AM, Ghanadian M, Afsharypuor S, Choudhary MI, Kobarfard F, Rahmati M (2010a) Two new lathyrane type diterpenoids from Euphorbia aellenii. Fitoterapia 81(7):891-893

Ayatollahi AM, Ghanadian M, Mesaik MA, Mohamed Abdella O, Afsharypuor S, Kobarfard F, Mirza-Taheri M (2010b) New myrsinane-type diterpenoids from Euphorbia aellenii Rech. f. with their immunomodulatory activity. J Asian Nat Prod Res 12(12):1020-1025

Bahmani B, Keyvanloo Shahrestanaki M, Ghanadian M, Hajiahmadi S, Aghaei M (2017) Jatropha-6 (17), 11 E-diene class derivatives induce apoptosis effects in OVCAR-3 and Caov-4 ovarian cancer cell lines via a mitochondrial pathway. Biochem Cell Biol 95(6):616-627

Barile E, Lanzotti V (2007) Biogenetical related highly oxygenated macrocyclic diterpenes from sea spurge Euphorbia paralias. Org Lett 9(18):3603-3606

Barile E, Fattorusso E, Ialenti A, Ianaro A, Lanzotti V (2007) Paraliane and pepluane diterpenes as anti-inflammatory agents: first insights in structure-activity relationships. Bioorg Med Chem Lett 17(15):4196-4200

Barile E, Borriello M, Di Pietro A, Doreau A, Fattorusso C, Fattorusso E, Lanzotti V (2008a) Discovery of a new series of jatrophane and lathyrane diterpenes as potent and specific P-glycoprotein modulators. Org Biomol Chem 6(10):1756-1762 
Barile E, Corea G, Lanzotti V (2008b) Diterpenes from Euphorbia as potential leads for drug design. Nat Prod Commun 3(6): 1003-1020

Bedoya LM, Márquez N, Martínez N, Gutiérrez-Eisman S, Álvarez A, Calzado MA, Rojas JM, Appendino G, Muñoz E, Alcamí J (2009) SJ23B, a jatrophane diterpene activates classical PKCs and displays strong activity against HIV in vitro. Biochem Pharmacol 77(6):965-978

Betancur-Galvis L, Checa J, Marco JA, Estornell E (2003) Jatrophane diterpenes from the latex of Euphorbia obtusifolia with inhibitory activity on the mammalian mitochondrial respiratory chain. Planta Med 69(02):177-178

Breitmaier E (2006) Terpenes: flavors, fragrances, pharmaca, pheromones. Wiley, London

Buey RM, Barasoain I, Jackson E, Meyer A, Giannakakou P, Paterson I, Mooberry S, Andreu JM, Díaz JF (2005) Microtubule interactions with chemically diverse stabilizing agents: thermodynamics of binding to the paclitaxel site predicts cytotoxicity. Chem Biol 12(12):1269-1279

Burkill HM (1994) The useful plants of west tropical Africa, vol 2. Families EI, Royal Botanic Gardens, New York

Callaghan R, Luk F, Bebawy M (2014) Inhibition of the multidrug resistance P-glycoprotein; time for a change of strategy? Drug Metab Dispos 113:056176

Chen H, Wang H, Yang B, Jin D-Q, Yang S, Wang M, Xu J, Ohizumi Y, Guo Y (2014) Diterpenes inhibiting NO production from Euphorbia helioscopia. Fitoterapia 95:133-138

Corea G, Fattorusso E, Lanzotti V, Taglialatela-Scafati O, Appendino G, Ballero M, Simon P-N, Dumontet C, Di Pietro A (2003a) Jatrophane diterpenes as P-glycoprotein inhibitors. First insights of structure-activity relationships and discovery of a New, powerful lead. J Med Chem 46(15):3395-3402

Corea G, Fattorusso E, Lanzotti V, Taglialatela-Scafati O, Appendino G, Ballero M, Simon P-N, Dumontet C, Di Pietro A (2003b) Modified jatrophane diterpenes as modulators of multidrug resistance from Euphorbia dendroides L. Bioorg Med Chem 11(23):5221-5227

Corea G, Fattorusso E, Lanzotti V, Motti R, Simon P-N, Dumontet C, Di Pietro A (2004a) Jatrophane diterpenes as modulators of multidrug resistance. advances of structure-activity relationships and discovery of the potent lead pepluanin A. J Med Chem 47(4):988-992

Corea G, Fattorusso E, Lanzotti V, Motti R, Simon P-N, Dumontet C, Di Pietro A (2004b) Structure-activity relationships for Euphocharacins A-L, a new series of jatrophane diterpenes, as inhibitors of cancer cell P-glycoprotein. Planta Med 70(07):657-665

Corea G, Fattorusso C, Fattorusso E, Lanzotti V (2005a) Amygdaloidins A-L, twelve new $13 \alpha-\mathrm{OH}$ jatrophane diterpenes from Euphorbia amygdaloides L. Tetrahedron 61(18):4485-4494

Corea G, Fattorusso E, Lanzotti V, Di Meglio P, Maffia P, Grassia G, Ialenti A, Ianaro A (2005b) Discovery and biological evaluation of the novel naturally occurring diterpene pepluanone as antiinflammatory agent. J Med Chem 48(22):7055-7062

Corea G, Di Pietro A, Dumontet C, Fattorusso E, Lanzotti V (2009) Jatrophane diterpenes from Euphorbia spp. as modulators of multidrug resistance in cancer therapy. Phytochem Rev 8(2):431-447

Dewick PM (2002) Medicinal natural products: a biosynthetic approach. Wiley, New York

Dobrev D, Friedrich A, Voigt N, Jost N, Wettwer E, Christ T, Knaut M, Ravens U (2005) Clinical perspective. Circulation 112(24):3697-3706

Duarte N, Lage H, Ferreira M-JU (2008) Three new jatrophane polyesters and antiproliferative constituents from Euphorbia tuckeyana. Planta Med 74(01):61-68

Dueber MT, Adolf W, West CA (1978) Biosynthesis of the diterpene phytoalexin casbene: partial purification and characterization of casbene synthetase from Ricinis communis. Plant Physiol 62(4):598-603

Eid SY, El-Readi MZ, Fatani SH, Eldin EEMN, Wink M (2015) Natural products modulate the multifactorial multidrug resistance of cancer. Pharmacol Pharm 6(03):146

El-Bassuony AA (2007) Antibacterial activity of new polyester diterpenes from Euphorbia guyoniana. Asian J Chem 19(6):4553

Engi H, Sakagami H, Kawase M, Parecha A, Manvar D, Kothari H, Adlakha P, Shah A, Motohashi N, Ocsovszki I (2006) Tumour-specific cytotoxicity and MDR-reversal activity of dihydropyridines. Vivo 20(5):637-643

Engi H, Vasas A, Redei D, Molnar J, Hohmann J (2007) New MDR modulators and apoptosis inducers from Euphorbia species. Anticancer Res 27(5A):3451-3458

Esposito ML, Nothias L-FL, Nedev H, Gallard J-FO, Leyssen P, Retailleau P, Costa J, Roussi F, Iorga BI, Paolini J (2016) Euphorbia dendroides Latex as a source of jatrophane esters: isolation, structural analysis, conformational study, and anti-CHIKV activity. J Nat Prod 79(11):2873-2882

Esposito ML, Nim S, Nothias L-FL, Gallard J-FO, Rawal MK, Costa J, Roussi F, Prasad R, Di Pietro A, Paolini J (2017) Evaluation of jatrophane esters from Euphorbia spp. as modulators of Candida albicans multidrug transporters. J Nat Prod 80(2):479-487

Evans F, Taylor S (1983) Pro-inflammatory, tumour-promoting and anti-tumour diterpenes of the plant families Euphorbiaceae and Thymelaeaceae. Prog Chem Org Nat Prod. Springer, Berlin, pp 1-99

Fakunle CO, Connolly JD, Rycroft DS (1989) The structure of enukokurin, a new jatrophane diterpenoid from the latex of Euphorbia lateriflora. J Nat Prod 52(2):279-283

Fang Y, Sun J, Zhong X, Hu R, Gao J, Duan G, Ji C, Chen L, Zhang W, Miao C (2018) ES2 enhances the efficacy of chemotherapeutic agents in ABCB1-overexpressing cancer cells in vitro and in vivo. Pharmacol Res 129:388-399

Fattorusso E, Lanzotti V, Taglialatela-Scafati O, Tron GC, Appendino G (2002) Bisnorsesquiterpenoids from Euphorbia resinifera Berg. and an expeditious procedure to obtain resiniferatoxin from its fresh latex. Eur J Org Chem 2002(1):71-78

Ferreira AMV, Carvalho LSH, Carvalho MJM, Sequeira MM, Silva AM (2002) Jatrophane and lathyrane diterpenoids from Euphorbia hyberna L. Phytochemistry 61(4):373-377

Ferreira M-JU, Gyémant N, Madureira AM, Tanaka M, Koos K, Didziapetris R, Molnar J (2005) The effects of jatrophane derivatives on the reversion of MDR1-and MRP-mediated 
multidrug resistance in the MDA-MB-231 (HTB-26) cell line. Anticancer Res 25(6B):4173-4178

Ferreira RJ, dos Santos DJ, Ferreira M-JU, Guedes RC (2011) Toward a better pharmacophore description of P-glycoprotein modulators, based on macrocyclic diterpenes from Euphorbia species. J Chem Inf Model 51(6):1315-1324

Ferreira M-JU, Duarte N, Reis M, Madureira AM, Molnár J (2014) Euphorbia and Momordica metabolites for overcoming multidrug resistance. Phytochem Rev 13(4):915-935

Flores-Giubi ME, Duran-Pena MJ, Botubol-Ares JM, EscobarMontaño F, Zorrilla D, Macías-Sánchez AJ, HernándezGalán R (2017) Gaditanone, a Diterpenoid Based on an Unprecedented Carbon Skeleton Isolated from Euphorbia gaditana. J Nat Prod 80(7):2161-2165

Geng D, Shi Y, Da Min Z, Liang JY (2010) A new diterpenoid from Euphorbia helioscopia. Chin Chem Lett 21(1):73-75

Ghanadian SM, Ayatollahi AM, Mesaik MA, Abdalla OM (2013) New immunosuppressive cyclomyrsinol diterpenes from Euphorbia kopetdaghi Prokh. Nat Prod Res 27(3):246-254

Ghanadian M, Saeidi H, Aghaei M, Rahiminejad MR, Ahmadi E, Ayatollahi SM, Choudhary MI, Bahmani B (2015) New jatrophane diterpenes from Euphorbia osyridea with proapoptotic effects on ovarian cancer cells. Phytochem Lett 12:302-307

Gottesman MM, Fojo T, Bates SE (2002) Multidrug resistance in cancer: role of ATP-dependent transporters. Nat Rev Cancer 2(1):48

Günther G, Hohmann J, Vasas A, Máthé I, Dombi G, Jerkovich G (1998) Jatrophane diterpenoids from Euphorbia esula. Phytochemistry 47(7):1309-1313

Guo J, Fang X, Di YT, Hua HM, Hao XJ (2010) Kansuinine J, a new macrocyclic diterpenoid from the roots of Euphorbia kansui. Chin Chem Lett 21(8):943-946

Haiming S, Zhida M, Pengfei T, Xiaobo L (2008) Chemistry and biological activity of diterpenoids from genus Euphorbia in China. Prog Chem 20(2/3):375

Hasan A, Liu G-Y, Hu R, Aisa H (2019) Jatrophane Diterpenoids from Euphorbia glomerulans. J Nat Prod 82:724-734

Hashimoto N, Yamashita T, Tsuruzoe N (2006) Tertiapin, a selective IKACh blocker, terminates atrial fibrillation with selective atrial effective refractory period prolongation. Pharmacol Res 54(2):136-141

Hegazy M-EF, Mohamed AE-HH, Aoki N, Ikeuchi T, Ohta E, Ohta S (2010) Bioactive jatrophane diterpenes from Euphorbia guyoniana. Phytochemistry 71(2-3):249-253

Hohmann J, Vasas A, Günther G, Máthé I, Evanics F, Dombi G, Jerkovich G (1997) Macrocyclic diterpene polyesters of the jatrophane type from Euphorbia esula. J Nat Prod 60(4):331-335

Hohmann J, Günther G, Vasas A, Kálmán A, Argay G (1999a) Isolation and Structure Revision of Pepluane Diterpenoids from Euphorbia peplus. J Nat Prod 62(1):107-109

Hohmann J, Vasas A, Günther G, Dombi G, Blazso G, Falkay G, Mathe I, Jerkovich G (1999b) Jatrophane diterpenoids from Euphorbia peplus. Phytochemistry 51(5):673-677

Hohmann J, Evanics F, Berta L, Bartók T (2000a) Diterpenoids from Euphorbia peplus. Planta Med 66(03):291-294
Hohmann J, Redei D, Evanics F, Kálmán A, Argay G, Bartók T (2000b) Serrulatin A and B, new diterpene polyesters from Euphorbia serrulata. Tetrahedron 56(22):3619-3623

Hohmann J, Evanics F, Dombi G, Molnár J, Szabó P (2001a) Euphosalicin, a new diterpene polyester with multidrug resistance reversing activity from Euphorbia salicifolia. Tetrahedron 57(1):211-215

Hohmann J, Evanics F, Dombi G, Szabó P (2001b) Salicifoline and salicinolide, new diterpene polyesters from Euphorbia salicifolia. Tetrahedron Lett 42(37):6581-6584

Hohmann J, Molnár J, Rédei D, Evanics F, Forgo P, Kálmán A, Argay G, Szabó P (2002) Discovery and biological evaluation of a new family of potent modulators of multidrug resistance: reversal of multidrug resistance of mouse lymphoma cells by new natural jatrophane diterpenoids isolated from Euphorbia species. J Med Chem 45(12):2425-2431

Hohmann J, Forgo P, Csupor D, Schlosser G (2003a) Isolation and structure determination of new jatrophane diterpenoids from Euphorbia platyphyllos L. Helv Chim Acta 86(10):3386-3393

Hohmann J, Rédei D, Forgo P, Molnár J, Dombi G, Zorig T (2003b) Jatrophane diterpenoids from Euphorbia mongolica as modulators of the multidrug resistance of L5128 mouse lymphoma cells. J Nat Prod 66(7):976-979

Hu R, Gao J, Rozimamat R, Aisa HA (2018) Jatrophane diterpenoids from Euphorbia sororia as potent modulators against P-glycoprotein-based multidrug resistance. European J Med Chem 146:157-170

Huang Y, Aisa HA (2010a) Jatrophane diterpenoids from fructus Euphorbia sororia. Phytochem Lett 3(4):176-180

Huang Y, Aisa HA (2010b) Three New Diterpenoids from Euphorbia sororia L. Helv Chim Acta 93(6):1156-1161

Iseki K, Sugawara M, Sato K, Naasani I, Hayakawa T, Kobayashi M, Miyazaki K (1999) Multiplicity of the H+ dependent transport mechanism of dipeptide and anionic $\beta$ lactam antibiotic ceftibuten in rat intestinal brush-border membrane. J Pharmacol Exp Ther 289(1):66-71

Jadranin M, Pešić M, Aljančić IS, Milosavljević SM, Todorović NM, Podolski-Renić A, Banković J, Tanić N, Marković I, Vajs VE (2013) Jatrophane diterpenoids from the latex of Euphorbia dendroides and their anti-P-glycoprotein activity in human multi-drug resistant cancer cell lines. Phytochemistry 86:208-217

Jakupovic J, Jeske F, Morgenstern T, Tsichritzis F, Marco J, Berendsohn W (1998a) Diterpenes from Euphorbia segetalis. Phytochemistry 47(8):1583-1600

Jakupovic J, Morgenstern T, Bittner M, Silva M (1998b) Diterpenes from Euphorbia peplus. Phytochemistry 47(8):1601-1609

Jakupovic J, Morgenstern T, Marco J, Berendsohn W (1998c) Diterpenes from Euphorbia paralias. Phytochemistry 47(8):1611-1619

Jassbi AR (2006) Chemistry and biological activity of secondary metabolites in Euphorbia from Iran. Phytochemistry 67(18): 1977-1984

Jin-Jun H, Yao S, Zhou Y, Lin F, Lu-Ying C, Shuai Y, Hua-Li L, Wan-Ying W, De-An G (2017) Anti-proliferation activity of terpenoids isolated from Euphorbia kansui in human cancer cells and their structure-activity relationship. Chin J Nat Med 15(10):766-774 
Kapoor L (2017) Handbook of Ayurvedic medicinal plants: Herbal reference library. Routledge, London

King AJ, Brown GD, Gilday AD, Forestier E, Larson TR, Graham IA (2016) A cytochrome P450-mediated intramolecular carbon-carbon ring closure in the biosynthesis of multidrug-resistance-reversing lathyrane diterpenoids. ChemBioChem 17(17):1593-1597

Kinghorn AD, Falk H, Kobayashi J (2011) Prog Chem Org Nat Prod. Springer, Berlin

Kirby J, Nishimoto M, Park JG, Withers ST, Nowroozi F, Behrendt D, Rutledge EJG, Fortman JL, Johnson HE, Anderson JV (2010) Cloning of casbene and neocembrene synthases from Euphorbiaceae plants and expression in Saccharomycescerevisiae. Phytochemistry 71(13):1466-1473

Kobayashi T, Ikeda K (2006) G protein-activated inwardly rectifying potassium channels as potential therapeutic targets. Curr Pharm Des 12(34):4513-4523

Kosemura S, Shizuri Y, Yamamura S (1985) Isolation and structures of euphohelins, new toxic diterpenes from Euphorbia helioscopia L. Bull Chem Soc Jpn 58(11):3112-3117

Krstić G, Jadranin M, Todorović NM, Pešić M, Stanković T, Aljančić IS, Tešević VV (2018) Jatrophane diterpenoids with multidrug-resistance modulating activity from the latex of Euphorbia nicaeensis. Phytochemistry 148:104-112

Kupchan SM, Sigel CW, Matz M, Saenz Renauld JA, Haltiwanger RC, Bryan RF (1970) Jatrophone, a novel macrocyclic diterpenoid tumor inhibitor from Jatropha gossypiifolia. JACS 92(14):4476-4477

Kusz N, Orvos P, Bereczki L, Fertey P, Bombicz P, Csorba A, Talosi L, Jakab G, Hohmann J, Redei D (2018) Diterpenoids from Euphorbia dulcis with potassium ion channel inhibitory activity with selective $G$ protein-activated inwardly rectifying ion channel (GIRK) blocking effect. J Nat Prod 81(11):2483-2492

Kúsz N, Orvos P, Csorba A, Tálosi L, Chaieb M, Hohmann J, Rédei D (2016) Jatrophane diterpenes from Euphorbia guyoniana are new potent inhibitors of atrial GIRK channels. Tetrahedron 72(37):5724-5728

Lanzotti V (2013) Diterpenes for therapeutic use. In: Natural products: phytochemistry, botany and metabolism of alkaloids, phenolics and terpenes, pp 3173-3191

Lanzotti V, Barile E, Scambia G, Ferlini C (2015) Cyparissins A and B, jatrophane diterpenes from Euphorbia cyparissias as Pgp inhibitors and cytotoxic agents against ovarian cancer cell lines. Fitoterapia 104:75-79

Lawant P, Winthagen D (2002) Euphorbia resinifera portrayed in a manuscript herbal nearly fifteen hundred years agorevisited. Bradleya 2002(20):13-17

Lee JW, Jin Q, Jang H, Lee D, Han SB, Kim Y, Hong JT, Lee MK, Hwang BY (2016) Jatrophane and ingenane-type diterpenoids from Euphorbia kansui inhibit the LPS-induced NO production in RAW 264.7 cells. Bioorg Med Chem Lett 26(14):3351-3354

Li P, Feng ZX, Ye D, Huan W, Da Gang W, Dong LX (2003) Chemical constituents from the whole plant of Euphorbia altotibetic. Helv Chim Acta 86(7):2525-2532

Li J, Li H-H, Wang W-Q, Song W-B, Wang Y-P, Xuan L-J (2018a) Jatrophane diterpenoids from Euphorbia helioscopia and their lipid-lowering activities. Fitoterapia 128:102-111

Li J, Wang W-Q, Tang S, Song W-B, Xuan L-J (2018b) Jatrophanes as promising multidrug resistance modulators: advances of structure-activity relationships. Fitoterapia 127:138-145

Liang Q-L, Dai C-C, Jiang J-H, Tang Y-P, Duan J-A (2009) A new cytotoxic casbane diterpene from Euphorbia pekinensis. Fitoterapia 80(8):514-516

Liu LG, Tan RX (2001) New Jatrophane Diterpenoid Esters from Euphorbia turczaninowii. J Nat Prod 64(8):1064-1068

Liu L, Meng J, Wu S, Li X, Zhao X, Tan R (2002) New macrocyclic diterpenoids from Euphorbia esula. Planta Med 68(03):244-248

Liu C, Liao Z-X, Liu S-J, Qu Y-B, Wang H-S (2014) Two new diterpene derivatives from Euphorbia lunulata Bge and their anti-proliferative activities. Fitoterapia 96:33-38

Lu Z-Q, Guan S-H, Li X-N, Chen G-T, Zhang J-Q, Huang H-L, Liu X, Guo D-A (2008) Cytotoxic diterpenoids from Euphorbia helioscopia. J Nat Prod 71(5):873-876

Lu D, Liu Y, Aisa HA (2014) Jatrophane diterpenoid esters from Euphorbia sororia serving as multidrug resistance reversal agents. Fitoterapia 92:244-251

Lv X-J, Li Y, Ma S-G, Qu J, Liu Y-B, Li Y-H, Zhang D, Li L, Yu S-S (2016) Antiviral Triterpenes from the Twigs and Leaves of Lyonia ovalifolia. J Nat Prod 79(11):2824-2837

Madureira AM, Gyémánt N, Ascenso JR, Abreu PM, Molnár J, Ferreira M-JU (2006) Euphoportlandols A and B, tetracylic diterpene polyesters from Euphorbia portlandica and their anti-MDR effects in cancer cells. J Nat Prod 69(6):950-953

Mai Z-P, Ni G, Liu Y-F, Li L, Shi G-R, Wang X, Li J-Y, Yu D-Q (2017a) Heliosterpenoids A and B, two novel jatrophanederived diterpenoids with a 5/6/4/6 ring system from Euphorbia helioscopia. Sci Rep 7(1):4922

Mai Z-P, Ni G, Liu Y-F, Li Y-H, Li L, Li J-Y, Yu D-Q (2017b) Secoheliosphanes A and B and secoheliospholane A, three diterpenoids with unusual seco-jatrophane and seco-jatropholane skeletons from Euphorbia helioscopia. J Org Chem 83(1):167-173

Mai Z-P, Ni G, Liu Y-F, Li L, Li J-Y, Yu D-Q (2018a) Heliojatrones $\mathrm{A}$ and $\mathrm{B}$, two jatrophane-derived diterpenoids with a 5/10 fused-ring skeleton from Euphorbia helioscopia: structural elucidation and biomimetic conversion. Org Lett 20(10):3124-3127

Mai Z, Ni G, Liu Y, Zhang Z, Li L, Chen N, Yu D (2018b) Helioscopianoids A-Q, bioactive jatrophane diterpenoid esters from Euphorbia helioscopia. Acta Pharm Sin B 8(5):805-817

Manners GD, Wong RY (1985) The absolute stereochemical characterization of two new jatrophane diterpenes from Euphorbia esula. J Chem Soc Perkin 1:2075-2081

Marco JA, Sanz-Cervera JF, Yuste A, Jakupovic J, Lex J (1996) Terracinolides A and B, two bishomoditerpene lactones with a novel carbon framework from Euphorbia terracina. J Org Chem 61(5):1707-1709

Marco JA, Sanz-Cervera JF, Yuste A, Jakupovic J (1997) Terracinolides from Euphorbia terracina. Phytochemistry 45(1):137-140

Marco JA, Sanz-Cervera JF, Yuste A, Jakupovic J, Jeske F (1998) Jatrophane derivatives and a rearranged jatrophane 
from Euphorbia terracina. Phytochemistry 47(8):1621-1630

Marco JA, Sanz-Cervera JF, Checa J, Palomares E, Fraga BM (1999a) Jatrophane and tigliane diterpenes from the latex of Euphorbia obtusifolia. Phytochemistry 52(3):479-485

Marco JA, Sanz-Cervera JF, Yuste A, Jakupovic J (1999b) Isoterracinolides $\mathrm{A}$ and $\mathrm{B}$, novel bishomoditerpene lactones from Euphorbia terracina. J Nat Prod 62(1):110-113

Miglietta A, Gabriel L, Appendino G, Bocca C (2003) Biological properties of jatrophane polyesters, new microtubuleinteracting agents. Cancer Chemother Pharmacol 51(1):67-74

Mongkolvisut W, Sutthivaiyakit S (2007) Antimalarial and antituberculous poly-O-acylated jatrophane diterpenoids from Pedilanthus tithymaloides. J Nat Prod 70(9):1434-1438

Mwine JT, Van Damme P (2011) Why do Euphorbiaceae tick as medicinal plants? a review of Euphorbiaceae family and its medicinal features. J Med Plant Res 5(5):652-662

Nakano Y, Ohtani M, Polsri W, Usami T, Sambongi K, Demura $\mathrm{T}$ (2012) Characterization of the casbene synthase homolog from Jatropha (Jatropha curcas L). Plant Biotechnol $12: 418$

Nim S, Mónico A, Rawal MK, Duarte N, Prasad R, Di Pietro A, Ferreira M-JU (2016) Overcoming multidrug resistance in Candida albicans: macrocyclic diterpenes from euphorbia species as potent inhibitors of drug efflux pumps. Planta Med 82(13):1180-1185

Nothias L-F, Boutet-Mercey S, Cachet X, De La Torre E, Laboureur L, Gallard J-FO, Retailleau P, Brunelle A, Dorrestein PC, Costa J (2017) Environmentally friendly procedure based on supercritical fluid chromatography and tandem mass spectrometry molecular networking for the discovery of potent antiviral compounds from Euphorbia semiperfoliata. J Nat Prod 80(10):2620-2629

Nothias-Scaglia L-F, Retailleau P, Paolini J, Pannecouque C, Neyts J, Dumontet V, Roussi F, Leyssen P, Costa J, Litaudon M (2014) Jatrophane diterpenes as inhibitors of chikungunya virus replication: structure-activity relationship and discovery of a potent lead. J Nat Prod 77(6):1505-1512

Nothias-Scaglia L-F, Dumontet V, Neyts J, Roussi F, Costa J, Leyssen P, Litaudon M, Paolini J (2015a) LC-MS2-Based dereplication of Euphorbia extracts with anti-Chikungunya virus activity. Fitoterapia 105:202-209

Nothias-Scaglia L-F, Gallard J-F, Dumontet V, Roussi F, Costa J, Iorga BI, Paolini J, Litaudon M (2015b) Advanced structural determination of diterpene esters using molecular modeling and NMR spectroscopy. J Nat Prod 78(10):2423-2431

Nothias-Scaglia L-F, Pannecouque C, Renucci F, Delang L, Neyts J, Roussi F, Costa J, Leyssen P, Litaudon M, Paolini J (2015c) Antiviral activity of diterpene esters on chikungunya virus and HIV replication. J Nat Prod 78(6): 1277-1283

Öksüz S, Gürek F, Yang S-W, Lin L-Z, Cordell GA, Pezzuto JM, Wagner H, Lotter H (1997) Paralinones A and B, novel diterpene esters from Euphorbia paralias. Tetrahedron 53(9):3215-3222

Palmeira A, Sousa E, Vasconcelos MH, Pinto MM (2012) Three decades of P-gp inhibitors: skimming through several generations and scaffolds. Curr Med Chem 19(13):1946-2025

Pan Q, Ip FC, Ip NY, Zhu H-X, Min Z-D (2004) Activity of Macrocyclic Jatrophane Diterpenes from Euphorbia kansui in a TrkA Fibroblast Survival Assay. J Nat Prod 67(9):1548-1551

Pattenden G, Smithies AJ (1996) Concise syntheses of cembrenes based on radical-mediated vinylcyclopropane ringopening reactions in casbene. J Chem Soc Perkin 1:57-61

Pesic M, Markovic J, Jankovic D, Kanazir S, Markovic I, Rakic L, Ruzdijic S (2006) Induced resistance in the human non small cell lung carcinoma (NCI-H460) cell line in vitro by anticancer drugs. J Chemother 18(1):66-73

Pešić M, Banković J, Aljančić IS, Todorović NM, Jadranin M, Vajs VE, Tešević VV, Vučković I, Momčilović M, Marković ID (2011) New anti-cancer characteristics of jatrophane diterpenes from Euphorbia dendroides. Food Cosmet Toxicol 49(12):3165-3173

Podolski-Renić A, Anđelković T, Banković J, Tanić N, Ruždijić S, Pešić M (2011) The role of paclitaxel in the development and treatment of multidrug resistant cancer cell lines. Biomed Pharmacother 65(5):345-353

Podolski-Renić A, Jadranin M, Stanković T, Banković J, Stojković S, Chiourea M, Aljančić I, Vajs V, Tešević V, Ruždijić S (2013) Molecular and cytogenetic changes in multi-drug resistant cancer cells and their influence on new compounds testing. Cancer Chemother Pharmacol 72(3):683-697

Rawal MK, Shokoohinia Y, Chianese G, Zolfaghari B, Appendino G, Taglialatela-Scafati O, Prasad R, Di Pietro A (2014) Jatrophanes from Euphorbia squamosa as potent inhibitors of Candida albicans multidrug transporters. J Nat Prod 77(12):2700-2706

Redei D, Hohmann J, Evanics F, Forgo P, Szabo P, Mathe I (2003) Isolation and structural characterization of new, highly functionalized diterpenes from Euphorbia serrulata. Helv Chim Acta 86(2):280-289

Rédei D, Forgo P, Molnár J, Szabó P, Zorig T, Hohmann J (2012) Jatrophane diterpenoids with multidrug resistancemodulating activity from Euphorbia mongolica Prokh. Tetrahedron 68(40):8403-8407

Rédei D, Boros K, Forgo P, Molnár J, Kele Z, Pálinkó I, Pinke G, Hohmann J (2015) Diterpene constituents of Euphorbia exigua L. and multidrug resistance reversing activity of the isolated diterpenes. Chem Biodivers 12(8):1214-1221

Rédei D, Kúsz N, Sátori G, Kincses A, Spengler G, Burián K, Barina Z, Hohmann J (2018) Bioactive segetane, ingenane, and jatrophane diterpenes from Euphorbia taurinensis. Planta Med 84(09/10):729-735

Reis M, Ferreira RJ, Serly J, Duarte N, Madureira AM, Santos DJVA, Molnar J, Ferreira M-JU (2012) Colon adenocarcinoma multidrug resistance reverted by Euphorbia diterpenes: structure-activity relationships and pharmacophore modeling. Anticancer Agents Med Chem 12(9):1015-1024

Reis MA, Paterna A, Ferreira RJ, Lage H, Ferreira M-JU (2014) Macrocyclic diterpenes resensitizing multidrug resistant phenotypes. Bioorg Med Chem 22(14):3696-3702

Reis MA, André VN, Duarte MT, Lage H, Ferreira M-JU (2015) 12, 17-cyclojatrophane and jatrophane constituents of Euphorbia welwitschii. J Nat Prod 78(11):2684-2690 
Reis MA, Ahmed OB, Spengler G, Molnár J, Lage H, Ferreira M-JU (2016) Jatrophane diterpenes and cancer multidrug resistance-ABCB1 efflux modulation and selective cell death induction. Phytomedicine 23(9):968-978

Remy S, Litaudon M (2019) Macrocyclic diterpenoids from Euphorbiaceae as A source of potent and selective inhibitors of chikungunya virus replication. Molecules 24(12):2336

Rinner U (2015) Progress in the preparation of jatrophane diterpenes. Eur J Org Chem 2015(15):3197-3219

Robert J, Jarry C (2003) Multidrug resistance reversal agents. J Med Chem 46(23):4805-4817

Robinson DR, West CA (1970) Biosynthesis of cyclic diterpenes in extracts from seedlings of Ricinus communis. II. Conversion of geranylgeranyl pyrophosphate into diterpene hydrocarbons and partial purification of the cyclization enzymes. Biochemistry 9(1):80-89

Schiff P, Horwitz SB (1980) Taxol stabilizes microtubules in mouse fibroblast cells. Proc Natl Acad Sci 77(3):1561-1565

Schnabel C, Hiersemann M (2009) Total synthesis of jatrophane diterpenes from Euphorbia characias. Org Lett 11(12):2555-2558

Schnabel C, Sterz K, Müller H, Rehbein J, Wiese M, Hiersemann M (2010) Total synthesis of natural and non-natural $\Delta 5,6 \Delta 12,13$-jatrophane diterpenes and their evaluation as MDR modulators. J Org Chem 76(2):512-522

Seip EH, Hecker E (1984) Derivatives of characiol, macrocyclic diterpene esters of the jatrophane type from Euphorbia characias. Phytochemistry 23(8):1689-1694

Sekine T, Kamiya M, Ikegami F, Qi J-F (1998) Structure of esulol A, a novel macrocyclic diterpene, from inner mongolian Euphorbia esula. Nat Prod Lett 12(3):237-239

Shadi S, Saeidi H, Ghanadian M, Rahimnejad MR, Aghaei M, Ayatollahi SM, Iqbal Choudhary M (2015) New macrocyclic diterpenes from Euphorbia connata Boiss. with cytotoxic activities on human breast cancer cell lines. Nat Prod Res 29(7):607-614

Shi Q-W, Su X-H, Kiyota H (2008) Chemical and pharmacological research of the plants in genus Euphorbia. Chem Rev 108(10):4295-4327

Shokoohinia Y, Chianese G, Zolfaghari B, Sajjadi S-E, Appendino G, Taglialatela-Scafati O (2011) Macrocyclic diterpenoids from the Iranian plant Euphorbia bungei Boiss. Fitoterapia 82(3):317-322

Shukla Y, Srivastava A, Kumar S, Kumar S (1999) Phytotoxic and antimicrobial constituents of Argyreia speciosa and Oenothera biennis. J Ethnopharmacol 67(2):241-245

Singla A, Kamla P (1990) Phytoconstituents of Euphorbia species. Fitoterapia 41(6):483-516

Sutherland CJ, Polley SD (2011) Genomic insights into the past, current and future evolution of human parasites of the genus Plasmodium. Genetics and evolution of infectious disease. Elsevier, New YorK, pp 607-635

Szakács G, Hall MD, Gottesman MM, Boumendjel AN, Kachadourian R, Day BJ, Baubichon-Cortay HLN, Di Pietro A (2014) Targeting the Achilles heel of multidrugresistant cancer by exploiting the fitness cost of resistance. Chem Rev 114(11):5753-5774
Tao H-W, Hao X-J, Liu P-P, Zhu W-M (2008) Cytotoxic macrocyclic diterpenoids from Euphorbia helioscopia. Arch Pharm Res 31(12):1547-1551

Torrance SJ, Wiedhopf RM, Cole JR, Arora SK, Bates RB, Beavers WA, Cutler RS (1976) Antitumor agents from Jatropha macrorhiza (Euphorbiaceae). II. Isolation and characterization of jatrophatrione. J Org Chem 41(10):1855-1857

Tsai J-Y, Rédei DR, Forgo P, Li Y, Vasas A, Hohmann J, Wu C-C (2016) Isolation of phorbol esters from Euphorbia grandicornis and evaluation of protein kinase $\mathrm{C}$-and human platelet-activating effects of euphorbiaceae diterpenes. J Nat Prod 79(10):2658-2666

Valente C, Ferreira MJ, Abreu PM, Pedro M, Cerqueira F, Nascimento MSJ (2003) Three new jatrophane-type diterpenes from Euphorbia pubescens. Planta Med 69(04):361-366

Valente C, Ferreira MJ, Abreu PM, Gyémánt N, Ugocsai K, Hohmann J, Molnár J (2004a) Pubescenes, jatrophane diterpenes, from Euphorbia pubescens, with multidrug resistance reversing activity on mouse lymphoma cells. Planta Med 70(01):81-84

Valente C, Pedro M, Ascenso JR, Abreu PM, Nascimento MSJ, Ferreira M-JU (2004b) Euphopubescenol and euphopubescene, two new jatrophane polyesters, and lathyranetype diterpenes from Euphorbia pubescens. Planta Med 70(03):244-249

Valente C, Pedro M, Duarte A, Nascimento MSJ, Abreu PM, Ferreira M-JU (2004c) Bioactive Diterpenoids, a New Jatrophane and Two e nt-Abietanes, and Other Constituents from Euphorbia pubescens. J Nat Prod 67(5):902-904

Valente IS, Reis M, Duarte NL, Serly J, Molnár JP, Ferreira M-JU (2012) Jatrophane diterpenes from Euphorbia mellifera and their activity as P-glycoprotein modulators on multidrug-resistant mouse lymphoma and human colon adenocarcinoma cells. J Nat Prod 75(11):1915-1921

Vasas A, Hohmann J (2014) Euphorbia diterpenes: isolation, structure, biological activity, and synthesis (2008-2012). Chem Rev 114(17):8579-8612

Vasas A, Sulyok E, Rédei D, Forgo P, Szabó P, Zupkó I, Berényi Á, Molnár J, Hohmann J (2011) Jatrophane diterpenes from Euphorbia esula as antiproliferative agents and potent chemosensitizers to overcome multidrug resistance. J Nat Prod 74(6): 1453-1461

Vasas A, Rédei D, Csupor D, Molnár J, Hohmann J (2012) Diterpenes from European Euphorbia species serving as prototypes for natural-product-based drug discovery. Eur Org Chem 2012(27):5115-5130

Vieira C, Duarte N, Reis MA, Spengler G, Madureira AM, Molnár J, Ferreira M-JU (2014) Improving the MDR reversal activity of 6, 17-epoxylathyrane diterpenes. Bioorg Med Chem 22(22):6392-6400

Villarreal AM, Dominguez XA, Williams HJ, Scott AI, Reibenspies J (1988) Citlalitrione, a new diterpene from Jatropha dioica var. sessiliflora. J Nat Prod 51(4):749-753

Wan L-S, Chu R, Peng X-R, Zhu G-L, Yu M-Y, Li L, Zhou L, Lu S-Y, Dong J-R, Zhang Z-R (2016a) Pepluane and paraliane diterpenoids from Euphorbia peplus with potential anti-inflammatory activity. J Nat Prod 79(6):1628-1634 
Wan L-S, Shao L-D, Fu L, Xu J, Zhu G-L, Peng X-R, Li X-N, Li Y, Qiu M-H (2016b) One-step semisynthesis of a segetane diterpenoid from a jatrophane precursor via a Diels-Alder reaction. Org Lett 18(3):496-499

Wang E-J, Casciano CN, Clement RP, Johnson WW (2000) Two transport binding sites of P-glycoprotein are unequal yet contingent: initial rate kinetic analysis by ATP hydrolysis demonstrates intersite dependence. Biochim Biophys Acta Protein Struct Mol Enzymol 1481(1):63-74

Wang L-Y, Wang N-L, Yao X-S, Miyata S, Kitanaka S (2002) Diterpenes from the roots of Euphorbia kansui and their in vitro effects on the cell division of Xenopus. J Nat Prod 65(9):1246-1251

Wang HY, Wang JS, Wei DD, Wang XB, Luo J, Yang MH, Kong LY (2012) Bioactivity-guided isolation of antiproliferative diterpenoids from Euphorbia kansui. Phytother Res 26(6):853-859

Wang J-X, Zheng L-L, Gao F, Zhou X-L (2019) Lewis acidmediated skeleton transformation of Euphorbia diterpenes: from lathyrane to euphoractane and myrsinane. Fitoterapia 133:212-218

Webster GL (1986) Irritant plants in the spurge family (Euphorbiaceae). Clin Dermatol 4(2):36-45

White RL, Burgess DS, Manduru M, Bosso JA (1996) Comparison of three different in vitro methods of detecting synergy: time-kill, checkerboard, and E test. Antimicrob Agents Chemother 40(8):1914-1918

Wiese M, Pajeva IK (2001) Structure-activity relationships of multidrug resistance reversers. Curr Med Chem 8(6):685-713

Wu C-P, Ohnuma S, Ambudkar SV (2011) Discovering natural product modulators to overcome multidrug resistance in cancer chemotherapy. Curr Pharm Biotechnol 12(4):609-620
Yamamura S, Shizuri Y, Kosemura S, Ohtsuka J, Tayama T, Ohba S, Ito M, Saito Y, Terada Y (1989) Diterpenes from Euphorbia helioscopia. Phytochemistry 28(12):3421-3436

Zhang W, Guo Y-W (2005) Three new jatrophone-type diterpenoids from Euphorbia helioscopia. Planta Med 71(03):283-286

Zhang W, Guo Y-W (2006) Chemical studies on the constituents of the chinese medicinal herb Euphorbia helioscopia L. Pharm Bull 54(7):1037-1039

Zhao X-R, Huo X-K, Dong P-P, Wang C, Huang S-S, Zhang B-J, Zhang H-L, Deng S, Liu K-X, Ma X-C (2015) Inhibitory effects of highly oxygenated lanostane derivatives from the fungus Ganoderma lucidum on P-glycoprotein and $\alpha$-glucosidase. J Nat Prod 78(8):1868-1876

Zhi-Qin S, Shu-Zhen M, Ying-Tong D, Xiao-Jiang H (2010) A new jatrophane diterpenoid from Euphorbia peplus. Chin J Nat Med 8(2):81-83

Zhou B, Wu Y, Dalal S, Cassera MB, Yue J-M (2016) Euphorbesulins A-P, Structurally Diverse Diterpenoids from Euphorbia esula. J Nat Prod 79(8):1952-1961

Zhu J, Wang R, Lou L, Li W, Tang G, Bu X, Yin S (2016) Jatrophane diterpenoids as modulators of P-glycoproteindependent multidrug resistance (MDR): advances of structure-activity relationships and discovery of promising MDR reversal agents. J Med Chem 59(13):6353-6369

Zolfaghari B, Yazdiniapour Z, Ghanadian M, Lanzotti V (2016) Cyclomyrsinane and premyrsinane diterpenes from Euphorbia sogdiana Popov. Tetrahedron 72(35):5394-5401

Publisher's Note Springer Nature remains neutral with regard to jurisdictional claims in published maps and institutional affiliations. 